\title{
Inhomogeneity in conduction and reentrant arrhythmias
}

Citation for published version (APA):

Lammers, W. J. E. P. (1987). Inhomogeneity in conduction and reentrant arrhythmias. [Doctoral Thesis, Maastricht University]. Rijksuniversiteit Limburg. https://doi.org/10.26481/dis.19870703wl

Document status and date:

Published: 01/01/1987

DOI:

10.26481/dis.19870703wl

Document Version:

Publisher's PDF, also known as Version of record

\section{Please check the document version of this publication:}

- A submitted manuscript is the version of the article upon submission and before peer-review. There can be important differences between the submitted version and the official published version of record.

People interested in the research are advised to contact the author for the final version of the publication, or visit the DOI to the publisher's website.

- The final author version and the galley proof are versions of the publication after peer review.

- The final published version features the final layout of the paper including the volume, issue and page numbers.

Link to publication

\footnotetext{
General rights rights.

- You may freely distribute the URL identifying the publication in the public portal. please follow below link for the End User Agreement:

www.umlib.nl/taverne-license

Take down policy

If you believe that this document breaches copyright please contact us at:

repository@maastrichtuniversity.nl

providing details and we will investigate your claim.
}

Copyright and moral rights for the publications made accessible in the public portal are retained by the authors and/or other copyright owners and it is a condition of accessing publications that users recognise and abide by the legal requirements associated with these

- Users may download and print one copy of any publication from the public portal for the purpose of private study or research.

- You may not further distribute the material or use it for any profit-making activity or commercial gain

If the publication is distributed under the terms of Article $25 \mathrm{fa}$ of the Dutch Copyright Act, indicated by the "Taverne" license above, 
Inhomogeneity in conduction

and

reentrant arrhythmias 
Voorplaat: 'Paal in stromend water"

ets van Johan Hemkes, 1966 (nr. 384)

met toestemming van de kunstenaar 


\title{
Inhomogeneity in conduction and reentrant arrhythmias
}

\author{
Academisch proefschrift
}

ter verkrijging van de graad van Doctor in de Geneeskunde aan de Rijksuniversiteit Limburg te Maastricht, op gezag van de Rector Magnificus, Prof. Dr. F.I.M. Bonke, volgens het besluit van het College van Dekanen in het openbaar te verdedigen op vrijdag 3 juli 1987 om 14.00 uur.

door

Willem Jacob Edoardo Petrus Lammers

geboren te Rome 
Promotores: Prof. Dr. M.A. Allessie, Maastricht

Prof. Dr. F.I.M. Bonke, Maastricht

Referenten: Prof. Dr. M.J. Janse, Amsterdam

Prof. Dr. H.J.J. Wellens, Maastricht

Prof. A.L. Wit, PhD, Columbia University, New York

This investigation was supported by the Foundation of Medical Research MEDIGON (Grant 900-516-091).

Financial support by the Netherlands Heart Foundation for the publication of this thesis is gratefully acknowledged. 
Most gracious King,

The Heart of all creatures is the foundation of their life, the Prince of all their parts, the sun of their microcosm, that on which all growth depends and from whence all strength and vigour flows. In like manner, the King is the foundation of his kingdom, the sun of his microcosm, the heart of his commonwealth, from whom all powers flows and all mercy proceeds. Those things that are here written about the movement of the heart, I have been the more bold to offer to your Majesty (as is the custom of this age), seeing that almost all human affairs are after the pattern of man and most affairs of a King are after that of the heart. Therefore the knowledgde of his own heart cannot be unprofitable to a King as being a divine example of his own actions (so have ever men been wont to comparate great things with small). Most royal Sovereign, placed on the pinnacle of all things human, here at least you may contemplate at one and the same time the principle of man's body and the image of your kingly power. Accept, therefore, I most humbly entreat your most gracious Majesty, with your wonted kindliness and indulgence, these new things concerning the heart, you who are the new splendor of this age and indeed its whole heart, a prince abounding in virtue and grace, to whom we acknowledge our thanks to be due for any good that our England receives or any joy that our life holds.

William Harvey: An anatomical disputation concerning the movement of the heart and the blood in living creatures, 1653. 


\section{CONTENTS}

Chapter 1: HISTORICAL INTRODUCTION

1.1 Conduction of the electrical impulse 1

1.2 Circus movement of the impulse 6

$\begin{array}{lr}1.3 \text { Inhomogeneity in excitability } & 15\end{array}$

$\begin{array}{lr}1.4 \text { Inhomogeneity in conduction } & 19\end{array}$

Chapter II: AIM OF THE STUDY 27

Chapter III: METHODS

3.1 Experimental techniques

3.1.1 Preparation 31

3.1.2 Superfusion 31

3.1.3 Stimulation $\quad 34$

3.2. Recording techniques $\quad 34$

3.2.1 Microelectrodes $\quad 34$

3.2.2 Electrophysiological mapping 35

- Electrodes $\quad 36$

- Amplifiers $\quad 36$

- Multiplexers and analog-digital conversion 38

- Tape recorder $\quad 38$

3.3 Data analysis $\quad 38$

3.4 Measurement of the effective refractory period 40

3.5 Measurement of the conduction velocity 40

Chapter IV: TACHY-ARRHYTHMIAS IN LOW-POTASSIUM

4.1 Introduction $\quad 43$

4.2 Nature of atrial arrhythmias induced in

4.3 Why is it easier to initiate intra-atrial
reentry in low-potassium?

4.4 Dimensions of intra-atrial reentry
in low-potassium

$\begin{array}{ll}4.5 \text { Discussion } & 56\end{array}$

Chapter V: TACHY-ARRHYTHMIAS IN HYPOXIA

5.1 Introduction $\quad 59$

5.2 Characteristics of atrial tachy-arrhythmias
in hypoxia

5.3 Mechanisms of the tachy-arrhythmias 62 
5.4 Why is it easier to initiate intra-atrial

reentry in hypoxia?

5.5 The effects of hypoxia on the transmembrane potential 71

5.6 Discussion

Chapter VI: SPATIAL DISPERSION IN REFRACTORINESS

AND CONDUCTION IN HYPOXIA

6.1 Introduction

6.2 Spatial dispersion of the refractory period

6.3 Quantification of conduction blocks

6.4 Discussion

Chapter VII: QUANTIFICATION OF TEMPORAL AND

SPATIAL INHOMOGENEITY OF CARDIAC EXCITATION

7.1 Introduction

7.2 Calculation of phase maps

7.3 Direction of propagation and inhomogeneity in conduction

7.4 The effect of prematurity on inhomogeneity in conduction

7.5 Pacing rate and inhomogeneity in conduction

7.6 Inhomogeneity in conduction and distance to the site of stimulation

7.7 Reentrant activity and inhomogeneity in conduction

7.8 Hypoxia and inhomogeneity in conduction

7.9 Discussion

Chapter VIII: GENERAL DISCUSSION

8.1 Minimal length of conduction block for the initiation of reentry

8.2 Size of the central arc of conduction block during sustained reentry

8.3 Size of conduction blocks and type of arrhythmia

8.4 Clinical significance of inhomogeneity in conduction

REFERENCES 



\section{Chapter I \\ HISTORICAL INTRODUCTION}

\subsection{Conduction of the electrical impulse.}

A study of the conduction properties in the heart is essentially a study of the electrical behavior of cardiac cells. Like all excitable tissues in the body, the quiescent heart cell displays an electrical potential gradient across its membrane and generates an action potential upon activation by neighboring cells. The action potential, in turn, is the signal for the interior of the heart cell to contract. Coordinated contraction of a large mass of cells, leading to well organized pumping of the heart, depends solely on the sequence of activation of the millions of individual cells. Disorganized conduction of the impulse will provoke disorganized or irregular contraction, disabling the pumping capacity of the heart and imperilling the human body.

Although since prehistoric days the heart has been known to contract rhythmically, and although the function of the heart as a pump for the blood circulation has been known since Harvey (1578), the investigation of the electrical phenomena in the heart is relatively recent. The presence of electrical energy in biological tissues was initially demonstrated in the skeletal muscle of the frog by Galvani (1791). This discovery was induced by numerous trials with muscles and nerves leading among others to the development of the nerve-muscle preparation by Swammerdam in 1665 (Lindeboom 1980), still popular in medical education programs. Such a preparation consists of a skeletal muscle, with its connecting nerve still attached, dissected free from the experimental animal, usually the frog. Upon electrical or mechanical stimulation of the nerve a contraction of the attached muscle can be observed. This principle was used by Kolliker and Muller (1855) to demonstrate the presence of electricity in the heart muscle. They placed the nerve end of the nerve-muscle preparation on top of the heart and obserwed a contraction of the connected muscle after each heart beat. They also made the observation "that the muscle of the limb contracted a scarcely perceptible time before the systole of the ventricle" (cited by Burdon-Sanderson and Page, 1879) making it clear that an 
electrical phenomenon preceded and induced the contraction wave. The nerve-muscle preparation was quite popular in its days and was used for many purposes including surprisingly a demonstration of circulating excitation. Kisseleff (1930) connected several nervemuscle preparations together in such a way that the nerve of one preparation rested on the muscle of the next preparation. The nerve of the last preparation, in turn, rested on the muscle of the first preparation to form a closed loop. Upon mechanicall stimulation of one nerve, they were able to set up a circulating sequence of activity, as shown by the regular sequence of contractions.

Much insight into the mechanism of the heart beat was gathered in the previous century despite the lack of electrical recording devices, the nerve-muscle preparation being too much a cumbersome equipment to detect reliably electrical currents. Most of this work was performed by observing and recording the contraction wave at specific sites as it followed the postulated electrical impulse. As described by Gaskell (1883): "The principle consists of registering the contractions of any two parts, the recording being effected by means of two levers attached by silk thread to the two parts of the heart." These levers were in turn connected to a recording drum to plot the contraction waves.

The propagation of contraction waves solved among others the fierce controversy between the myogenic and the neurogenic mechanism of the heart. Because nervous ganglia were found at the site of initiation of the cardiac impulse (by Remak in 1844) and at the junction between the auricles and the ventricles (by Bidder in 1852) it seemed logical to conclude that conduction in the heart was a property of nervous tissue. Furthermore, Kronecker and Schimey (1884) reported" a "sehr merkwiirdiger Phänoment" "Der eine von uns senkte eine Nadel in das Herz ... Da hörten, momentan gelämht, die Herzkammern zu pulsiren auf und verfielen, diastolisch erweitert, in fibrilläre Zuckungen, wie man solche bei acut absterbenden Herzen zu sehen pflegt ... Das Tier starb, ohne dass die Kammern noch einen Schlag ausgefiunt hatten." They conclude that "Hier - am geschüztesten Orte des Herzens - muss also ein Kreuzungspunkt der Innervationswege liegen, welcher in der Norm als Coordinationscentrum fiur die Musculatur der Herzkammern dient und wirksame Pulse ermöglicht". But Gaskell and Engelmann demonstrated that conduction through muscle cells was possible and thereby established the myogenic conduction property of the heart. Gaskell (1883) removed strips of tissue from the tortoise ventricles, devoid of nervous structures, and showed the existence of spontaneous rhythms and of impulse conduction in those strips. Engel- 

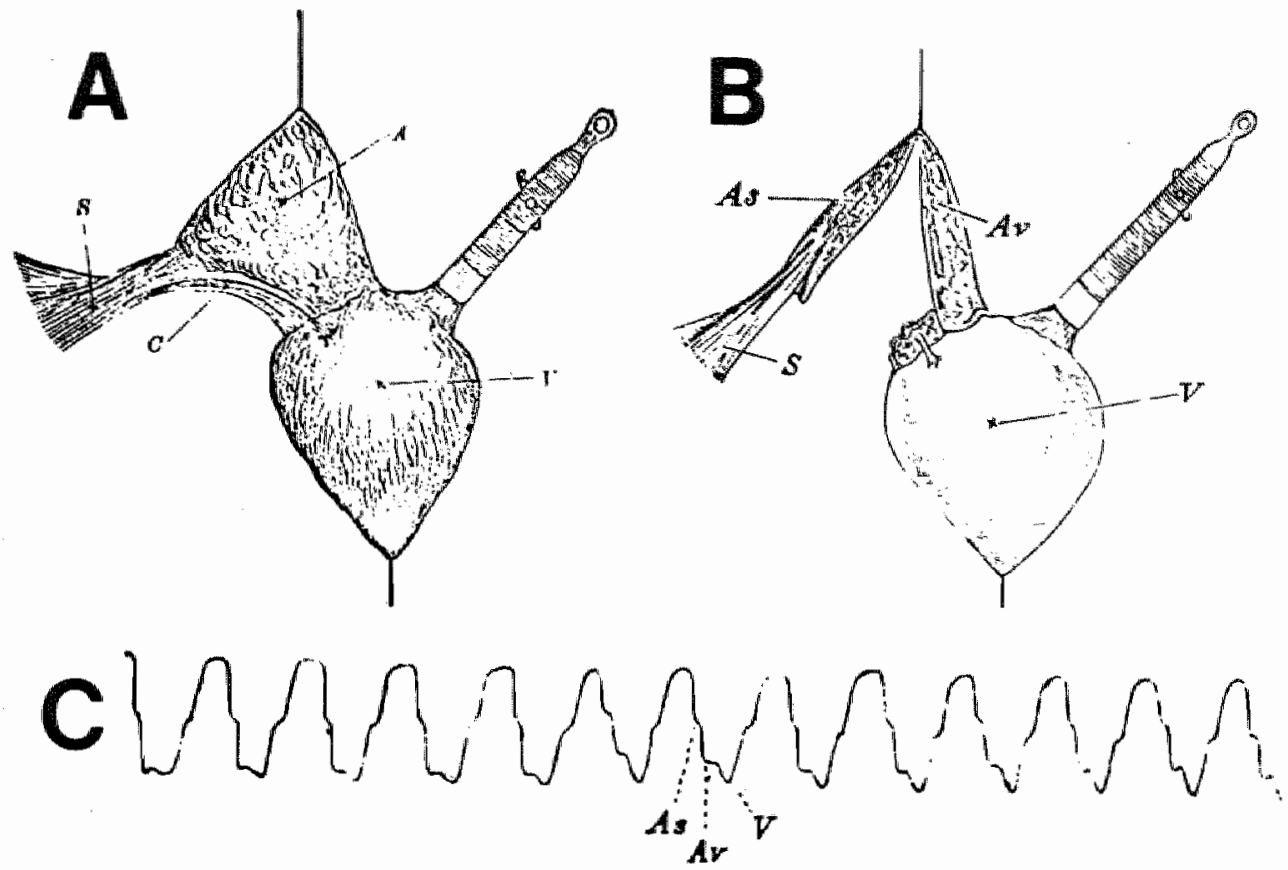

Figure 1.1. Preparation of the tortoise heart as used by Gaskell (1883) in his studies on partial conduction block. Panel A depicts the whole preparation consisting of sinus tissue (S), atria (A) and ventricles (V). Two silk ligatures have been attached to the atria and the ventricles to record the contraction waves. In panel $B, a$ partial block was created by cutting through the atria leaving a thin strand between the two parts (As and Av) intact. As shown in the tracing recorded from the atrium (panel $C$ ), slow conduction through the strand induced two distinct separate contraction waves (As and $A w$ ) in the recording.

mann's elegant experiments (1874, 1875) consisted of cutting a frog's ventricle by a zigzag cut which severed all nerve fibers running through the muscle without interrupting muscular continuity. Upon stimulation of any part of the muscle strand, he allways observed the passage of the contraction to all connected parts. Engelmann also made the first attempt to calculate conduction velocities. By simply measuring time and distance, he found a value 
of $0.02 \mathrm{~m} / \mathrm{s}$, but warned that this might be a low value due to the extensive injury to the tissue. Demonstration of myogenic conduction in the mammalian heart was performed by Porter (1899) who repeated Engelmann's technique in the cat and dog heart and concluded that: "there can be no doubt that the mammalian and the frog heart are alike in that a very small bridge of cardiac tissue suffices to carry the excitation wave from one part of the heart to the neighboring parts".

Not only did these studies conclusively show that propagation of the impulse was a property of the myocardium itself, it was also realized that discontinuities in the heart muscle could impair its conduction properties. Romanes (1855), in his work on the passage of the contraction waves in the medusae, coined the term "block" to express any hindrance to the passage of the contraction wave. Gaskell (1883) introduced the term in his studies on conduction in the tortoise heart. He could also vary the degree of block: "The application of 0.75 p.c. solution of salt to the blocking point is very efficacious in removing this partial block ... and then by simply drying with a piece of blotting paper the block can be brought back." In figure 1.1, the method of "partial block" is depicted by which he could demonstrate slow conduction as shown in the tracing of the contraction wave (panel C).

Meanwhile, various attempts were made to record directly the electrical phenomena of the heart. The first succesfull instrument was the differential rheotome, developed by Bernstein in 1868 (Fournier 1976), making it possible to measure the flow of current between two sites of the heart muscle. With this instrument, Burdon-Sanderson and Page (1879) investigated the conduction process in the heart of the frog. In this paper they start with an eulogy to Engelmann": "We owe most to the labours of Engelmann, whose researches on the propagation of the wave of contraction in the ventricle, on the electromotive properties of the resting heart, and on the electrical changes which immediately follow excitation, leave little more to be done". But in the same paper they pointed

1 Johannes Brahms dedicated his third string quartet to Engelmann (the first two were dedicated to Billroth). In 1890, Billroth wrote prophetically to Engelmann: "I'm afraid these dedications will keep our names longer in memory than the best work we have done. For us, not very complimentary, but beautiful for humanity, which, with the proper instinct, considers art more immortal than science. It is a human fact that love counts more than great respect". 
out that measurement of conduction velocities, as performed by their predecessors, may be gravely in error. "We found that correct results cannot be obtained by comparing the distance between the seat of excitation and any point in its progress, with the time at which its arrival at that point is indicated galvanometrically, for the reason that, neither the time nor the place of starting can be determined with certainty. When an induction current passes through a tissue, its excitatory infuence extend over an undefined area which surrounds the contacts. The excitation wawe starts from the edge of this area, and consequently at an unknown distance from the electrodes". And they conclude that: "the only satisfactory way of measuring the rate of transmission is to compare the difference between the times at which the excitation wave attains two points in a straight line emanating from the seat of excitation, with the distance between these points." This has become the standard method for measuring conduction velocities in heart muscle and was also used in this thesis (paragraph 3.5).

The rheotome was a tedious instrument to work with, requiring several persons to attend to its mechanics. Furthermore, it was only possible to measure the currents at predetermined moments. To obtain a full record of the changes in current during the passage of the impulse at a single site, many measurements had to be made sequentially and the experiment had to be repeated several times (Hoff and Geddes, 1957). The capillary-electrometer, developed by Lippmann in 1873, made it possible to record the electrical current continuously. It consisted essentially of a glass capillary tube filled at one end with mercury and at the other end with diluted sulphuric acid (de Waart, 1957). When a weak electric current passes through this system, the surface tension of the mercury changes, causing the meniscus to move in the tube in proportion to the applied current strength. An adaptation by Marey (1876) allowed the microscopic variations of the meniscus to be magnified and recorded by projecting a light through the capillary and a series of lenses on a photographic plate. With this recording device, BurdonSanderson and Page (1883) noted that local pressure interferes with normal propagation in the heart muscle. In the heart of a tortoise, a cut was made dividing the ventricles in an upper and a lower part connected to each other through a small connecting bridge. At the site of the bridge, a transitory block could be produced by local pressure. "The effect of the pressure was ... to obstruct completely the propagation of the excitatory wave, i.e. the auricular contraction was followed by a contraction of the part of the ventricle on the auricular side of the block - the rest of the 
ventricle remaining relaxed and motionless".

The disadvantage of the capillary-electrometer was its slow response time, making it impossible to study rapid electrical phenomena. It was this handicap which prompted Einthoven to develop a much faster responding system, the string galvanometer which was first presented in 1901. The string galvanometer essentially consisted of a silvered quartz string, with a thickness of 2 microns, stretched between the poles of an electromagnet (Einthoven, 1901). When a current is applied to the string, the string will deflect in proportion to the applied current. These small movements were, like in the capillary-electrometer, magnified and projected on a moving photographic plate. With this device, both the electrocardiogram, recorded from the surface of the body, and electrograms recorded from the surface of the heart, became available. The introduction of this fast and reliable recording apparatus marks the starting point of a new era in the study of the electrical behavior of the heart.

\subsection{Circus movement of the impulse.}

The study of cardiac impulse conduction in the previous century, and the establishment of myogenic conduction, was not an esoteric academic exercise. Several investigators realized that abnormal conduction in the heart may play a crucial role in the genesis of severe arrhythmias of the heart, especially of fibrillation. The first experimental demonstration of ventricular fibrillation was performed by Erichsen in 1842, who noticed the occurrence of "tremulous actions" after ligation of the coronary arteries (Snellen, 1984). Hoffa and Ludwig (1850) were the first to study ventricular fibrillation more systematically. They found that strong faradic stimulation invariably induced fibrillation: "Wenn eine sehr kräftige elektrische Wirkung ein recht reizbares Herz trifft ... geräht das Herz in ausserordentlich rasche, ganz unregelmässige Bewegungen von sehr geringer Intensität. Die Unregelmässigkeit dieser Bewegungen entsteht dadurch, dass die einzelnen anatomischen Elemente sich aus ithren Beziehungen zu einander lösen und die Gleichzeitigkeit ihrer Kontraktion aufgeben". MacWilliam (1887) introduced the concept of reexcitation during fibrillation: "The peristaltic contraction travelling along such a structure as the ventricular wall must reach adjacent muscle bundles at different points of time, and since these bundles are connected with one another by anastomosing branches, the contraction would naturally be propagated from one contracting fiber to another over which the contraction wave had 
already passed". Porter (1899) added to this concept the hypothesis of conduction blocks: "The appearance of such a spasm at several points might break up the normal conduction of the contraction wave by interposing here and there regions, the intense spasmodic contraction of which would block the passage of the contraction wave, just as it is blocked experimentally by squeezing the muscle fibers together with a clamp, and would leave the remaining muscle fibers dissociated and in confusion." Porter (1899) further demonstrated that, regardless whether fibrillation was initiated by electrical, mechanical or chemical means and regardless of the species in which it was evoked, the mechanism of fibrillation was essentially similar.

However, the study of reexcitation was not initiated in the heart muscle at all, but on the jelly-fish. Mayer (1906) removed the marginal sense-organs of the scyphomedusa to obtain a complete ring of excitable tissue: "Then upon momentarily stimulating the disk in any manner, it suddenly springs into rapid, rhythmical pulsation so regular and sustained as to recall the movement of clockwork". Two years later, Mayer (1908) demonstrated that these regular rhythmical pulsations were caused by a continuously circulating impulse in the ring. In his description of the mode of initiation of the circus movement however, Mayer made an interesting mistake, shown in figure 1.2. In the upper panel, a stimulus is applied at the dot initiating two wavefronts which collided against each other at the opposite site in the ring, thereby "annulling the pulsation". In the lower panel, two impulses of unequal magnitude are initiated. Upon meeting at the other side of the ring, Mayer believed that the strong impulse would override the weak contraction, stimulate the tissue over which the weak wave had just travelled, and thereby initiate a circulating excitation. Clearly, Mayer had no idea of the concept of the refractory period. The refractory period was initially discovered by Marey (1876) and implicates that an electrical impulse is always followed by a period of inexcitability. Engelmann (1893) demonstrated that the period of absolute refractoriness is followed by a period of relative refractoriness, during which a new impulse can be evoked to propagate at a slower speed. These concepts were correctly integrated in the theory of circus movement by Mines and Garrey. Mines (1913, 1914) made ring preparations of the hearts of tortoise (figure 1.3, upper panel), dogfish, dog and cats. In all these preparations he could initiate circulating excitation, the principles of which are shown in figure 1.3 (lower panel). "Supposing an excitation to be started in such a closed circuit and supposing that for some reasons it travels 

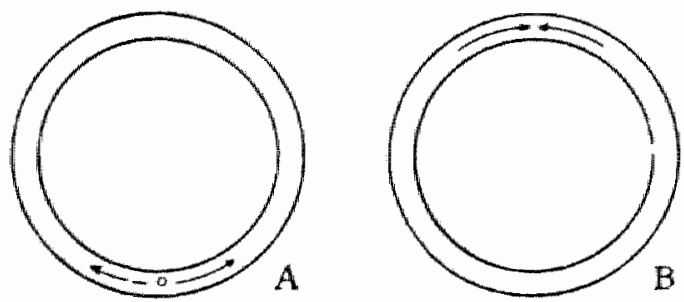

-Annulling of pulsation which occurs

when two waves of equal magnitude, coming in opposite directions, meet each other.
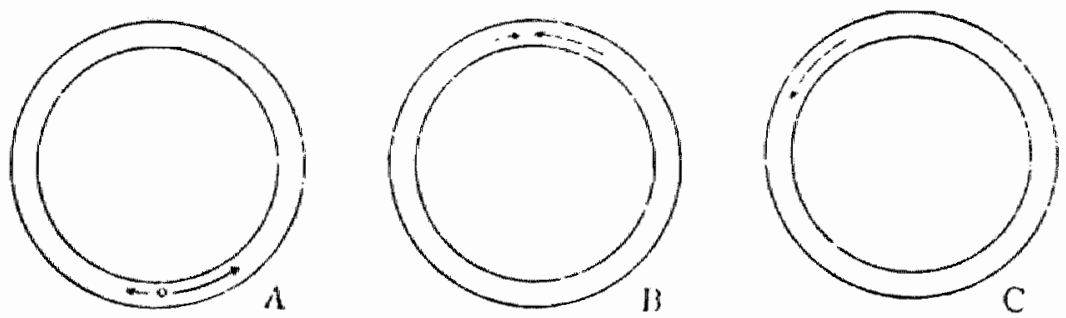

-Showing that when a strong wave meets a weak one it suppresses the weak wave, and remains the only wave in the circuit.

Figure 1.2. Diagrams from Mayer (1908) of a ring of excitable cells from the jelly-fish, illustrating his erroneous concept of the initiation of $a$ circulating excitation. In the upper panel, two contraction waves of equal amplitude are initiated at the dot, which cancel each other out at the other side of the ring. Upon induction of two unequal waves (lower panel), the strong wave was thought to "override" the weak one, and start a circus movement.

in one direction but not in the other. If the rate of propagation is rapid as compared with the duration of the wave, the whole circuit will be in the excited state at the same time, and the excitation will die out (see figure 1.3a). But if, on the other hand, the wave is slower and shorter (and it is made slower and shorter by the conditions which produce fibrillation) the excited state will have passed off at the region where the excitation started before the wave of excitation reaches this point on the circle at the completion of its revolution ... Under these circumstances, the wave of 

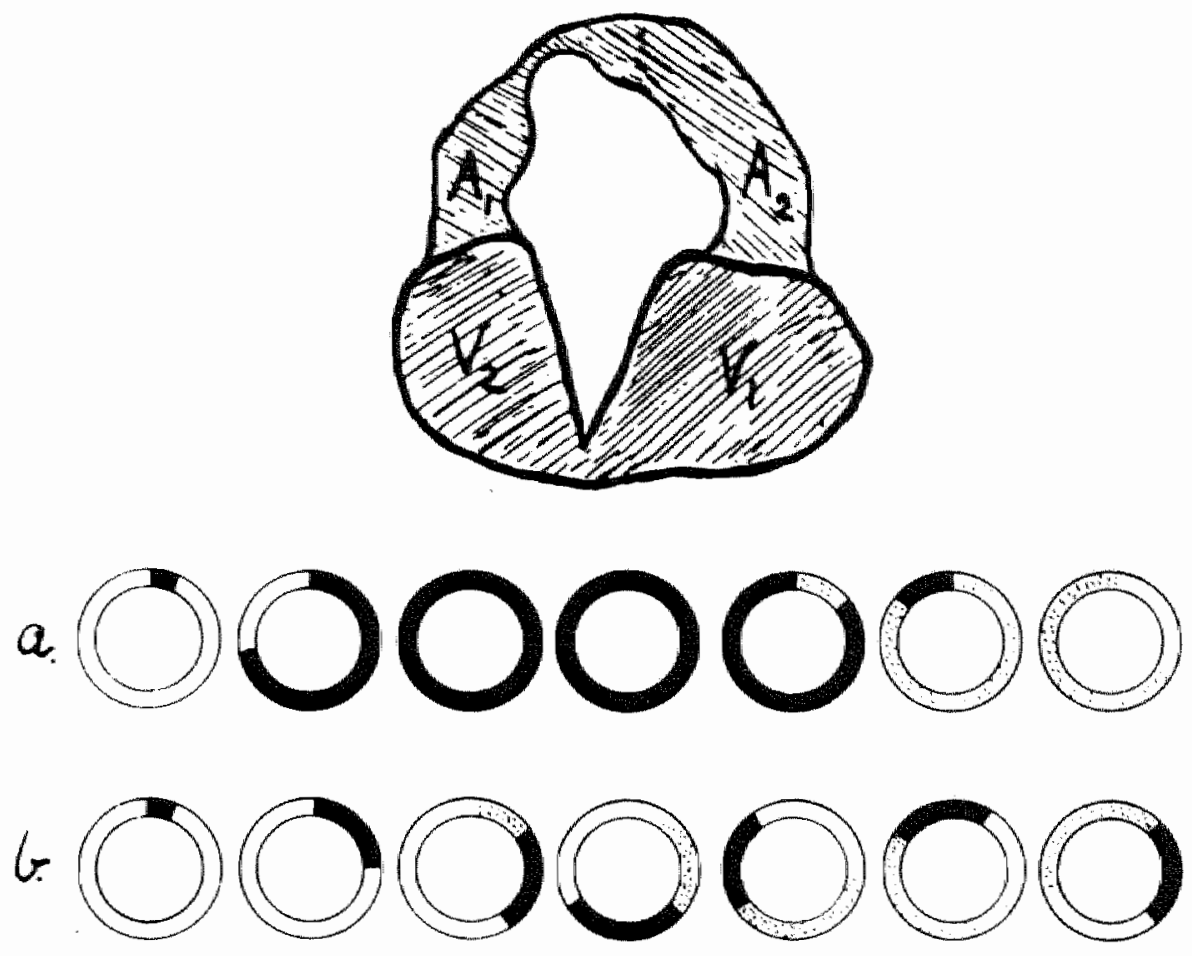

Figure 1.3. The upper panel is a sketch by Mines (1913) of a tortoise heart dissected into a ring. During a circulating excitation, the four parts of the heart contracted in the sequence VI, V2, $A 1$ and A2. The lower panel depicts his concepts of the initiation of circus movement. The absolute and the relative refractory periods are indicated by the black respectively by the dotted area. Fully excitable tissue is indicated in white. See text for further descrip. tion.

excitation may spread a second time over the same tract of lissue (see figure 1.3b): once started in this way it will continue unless interfered with by some external stimulus."

In this brilliant description by Mines, two important concepts are present which need further digression: the length of the circulating wave and the unidirectional block. If the length of the circulating impulse is too long, the advancing wavefront will at a certain moment encounter inexcitable tissue and the impulse will die out (figure 1.3a). This "wavelength" is determined both by the duration of the refractory period and by the conduction velocity. Shortening 
of the refractory period or slowing of conduction will both decrease the length of the impulse, making reexcitation possible (figure 1.3b). Garrey (1914) contributed to this concept by demonstrating that fibrillation, which was correctly thought by Mines and Garrey to be caused by circulating excitations, was very easily produced in large hearts, but impossible upon decreasing the mass of available tissue below a critical value. Since these pioneering studies, both slow conduction and short refractory period have been attributed to play an essential role in the mechanism of circus movement arrhythmias. Interest in the wavelength has revived in recent years (Wiener and Rosenblueth 1946, Smeets 1983, Smeets et al. 1986, Rensma 1987). Rensma measured the wavelength of the impulse in the atria of conscious dogs and showed that the wavelength was a better predictor for the inducibility of atrial flutter and fibrillation than conduction velocity or refractory period alone.

The second concept introduced by Mines relates to the occurrence of local conduction block. To set up a circus movement, the impulse should propagate in one direction and should block in the other direction, as shown in figure 1.3. Garrey (1914), in rings of the turtle heart, especially noted the spontaneous occurrence of blocks which: "affected the contraction waves passing in one direction only; those moving in the opposite direction passed the region of block and continued their progress about the ring" and "The causes underlying the unidirectional selection by such blocks is not altogether clear ... (but) the fact remains that spontaneous blocks of this type do appear, and, in our opinion, this fact is of greatest importance for any adequate theory of fibrillation". "Thus, the term "uni-directional conduction block" was coned. Mimes "nau a very clear opinion on the origin of conduction blocks. He considered differences in refractory period to be responsible (1913): "A slight difference in the rate of recovery of two divisions of the A-V connexion might determine that an extrasystole of the ventricle, provoked by a stimulus applied to the ventricle shortly after activity of the $A-V$ connexion, should spread up to the auricle by that part of the A-V connexion having the quicker recovery process and not by the other part". Mines also stressed that early stimulation after a previous excitation may induce fibrillation, thereby reinforcing the notion that fibrillation essentially consists of circulating excitations (1914): "... the critical instant for the production of fibrillation is immediately after the close of the refractory phase .... the stimulus apparently arrives at some part of the ventricular muscle just at the end of the refrac- 
tory phase and probably before the refractory has ended in some other regions of the muscle".

As described in the previous paragraphs, the work of Mayer, Mines and Garrey established the possibility of circulating excitations around anatomical obstacles in the heart. Lewis et al. (1920) demonstrated the presence of circus movement in atrial flutter in the dog heart, by mapping the sequence of activity during the arrhythmia with a double string-galvanometer. They concluded that the impulse rotated around the orifices of the superior and inferior caval veins. Rosenblueth and Garcia Ramos (1947), crushed the tissue between the orifices of the caval veins, thereby extending the size of the central obstacle and increasing the chances for initiation and perpetuation of atrial flutter. With the studies of Rosenblueth and Garcia Ramos (1947), Kimura et al. (1954), Lanari et al. (1956) and Hayden et al. (1967), the principles of circus movement around anatomical obstacles (figure 1.4) were developed. These principles are a fixed localization and length of the circuit, an excitable gap between head and tail of the impulse and a rate of the arrhythmia determined by the conduction velocity of the impulse and the length of the circuit.

The first demonstration of circus movement around an anatomical obstacle in humans was performed by Durrer and coworkers (1967a, 1967b) who studied the pattern of activity in patients suffering from the Wolff-Parkinson-White syndrome. In these patients, an accessory bundle exists between the atrium and the ventricle (Kent 1913). By epicardial mapping and programmed electrical stimulation, Durrer et al. demonstrated that the impulse propagated in a circuitous pathway involving the atria, the normal AV connection, the ventricles and back to the atria through the accessory pathway (for reviews see Durrer and Wellens 1974, Wellens 1975b, Wellens et al. 1980, 1984). Mines $(1913,1914)$ had predicted this arrhythmia, as soon as Kent discovered the accessory bundle, and proposed the ultimate test to establish the presence of circus movement. Cutting the circuit at any point should stop the arrhythmia. Since the advent of open heart-surgery, this test is succesfully used to cure circus movement tachycardias in WPW patients.

Another type of circus movement, without the involvement of a central anatomical obstacle, was demonstrated by Allessie et al. (1973, 1976, 1977). In retrospect, speculations about the existence of circus movement without an anatomical obstacle had already been made by several authors. Garrey (1914) discussed the possibility that: "... natural rings are not essential for maintenance of circus contractions". Lewis (1925, chapter 27$)$, in a classic piece of 


\section{CIRCUS MOVEMENT}

\section{Anatomically determined}

(Mines, 1913)

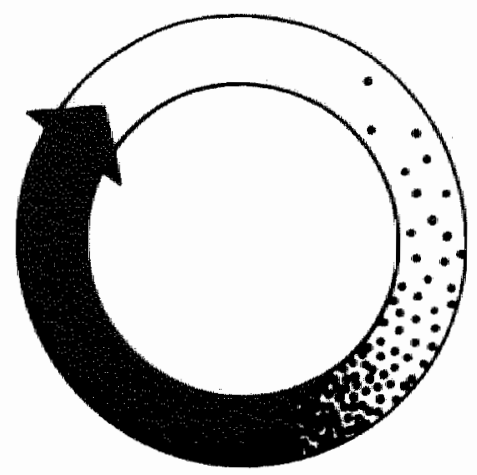

1. Fixed length and localization of circuit.

2. Circuit length equal to anatomical pathway.

3. Excitable gap between head and tail of impulse.

4. Rate proportionall to conduction velocity and length of pathway.
Functionally determined (Allessie et al. 1977)

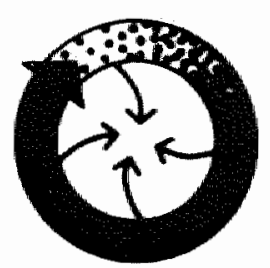

1. Variable size and localization of circuit.

2. Circuit length equal to the length of the excitation wave.

3. No gap of full excitability.

4. Rate proportional to refractory period.

Figure 1.4. The characteristics of anatomically and functionally determined circus movements. 
arm-chair physiology, described some of the properties of functional circus movement: "The conditions existing in the circle of muscle in which the gap is shortest ... are those which determine the rate of beating of the muscle sheet as a whole. That is to say, the rate of beating is not controlled by the length of the path, providing sufficient optional paths are available, for the length of path is determined by the remaining factors. It is probable, too, that the rate of conduction in these circumstances would not influence the rate of beating since a change in conduction would at once be balanced by an appropriate change in the length of path followed" and "it would seem that the length of the refractory period is in sole control of the rate of beating". In a review, Dawes (1952) clearly states the possibility of the absence of an anatomical obstacle during reentrant arrhythmias in some of his experiments but never published his results. West and Cox (1960) induced tachy-arrhythmias in the isolated right atrium of the rabbit in the presence of low potassium and acetylcholine. They demonstrated that the rate of the arrhythmia was dependent on the refractory period while a reduction of the tissue mass below a critical value abolished the arrhythmias, indirect evidence pointing to the existence of functional circus movement.

The studies of Allessie et al. (1977), performed in the isolated rabbit atrium, have determined the principles of functional circus movement (figure 1.4): a) the absence of an anatomical obstacle, by no excitable gap between crest and tail of the circulating impulse, c) a circuit length completely determined by the wavelength (being the product of the refractory period and the conduction velocity), d) a rate determined solely by the refractory period and e) variable size and localization of the circuit upon variation of the electrophysiological properties (Allessie and Bonke 1980). These principles of functional circus movement were incorporated into a "leading circle" model (Allessie et al. 1977). Recently Allessie et al. (1984) demonstrated that rapid but unstable atrial flutter, in the Langendorff perfused canine heart, was caused by functional circus movement. Boyden and Hoffman (1981) demonstrated flutter based on the "leading circle" model in dogs with right atrial hypertrophy. Page et al. (1983) described functional reentry in atrial flutter which was induced by sterile pericarditis.

Although to this date the precise mechanism of atrial flutter in patients has not been directly demonstrated, there are reasons to believe that both models do not apply to human atrial flutter. Conduction obstacles have been shown to exist in patients (Legato et al. 1974), but are seldom of the size necessary for a full size 
anatomical circuit. On the other hand, the absence of an excitable gap in the "leading circuit" model makes this type of arrhythmia unstable, which is in contrast with the well known persistence and stability of atrial flutter in patients. To obtain a situation in which a circus movement exists for longer periods of time, some degree of excitable gap, possibly combined with a small obstacle must be present.

The two types of circulating excitations should be regarded as two extremes of a wide spectrum of reentrant arrhythmias. In figure 1.5 several examples of reentrant arrhythmias are given. One extreme is the circulating excitation around a large anatomical obstacle and a fully recovered excitable gap as described by Mines (panel A). The other extreme is the smallest possible circuit, without an excitable gap and without an anatomical obstacle as described in the "leading circle" model (panel D; Allessie et al. 1977). The model of Lewis (panel B) consists of a circuit revolving around two small obstacles (like the caval orifices) with a functional conduction block bridging the two obstacles. The model of Moe (panel C) takes the differences in conduction velocity into account (Pastelin et al. 1978, Moe et al. 1980). In this model, the circuit is determined by a ring of fast conducting tissue, surrounding an area with slow or depressed conduction. Panell E depicts a model of a small circuit with a partially excitable gap revolving around a single but small obstacle, like the tricuspid valve (Frame et al. 1986). In panel $F$, an area of depressed conduction is assumed between an internal obstacle and the atrioventricular ring while in panel $G$, an area of prolonged refractoriness neighbors an anatomical obstacle. In both models, the slow conduction in part of the circuit creates an excitable gap which makes these circuits smaller and more stable. In the model presented in panel $H$, no gross anatomical obstacle is present. However the difference with the "leading circle" model (panel D) is the fact that the circuit is revolving in an anisotropic medium, implicating that conduction parallel to the fiber orientation is faster than conduction across the fibers (Spach et al. 1982, 1986a). This will change the circuit into an ellipsoidal shape, whereby the impulse revolves along a line of conduction block parallel to the fiber orientation (Dillon et al. 1985). An experimental model of this type of "anisotropic reentry" has been recently developed (Allessie et al. 1987) demonstrating, among others, that the tachycardias based on this type of reentry are very stable. Such a stability can only be accounted for by the presence of an excitable gap in the circuit. Computer simulations of such anisotropic circuits have revealed that an excitable gap is 

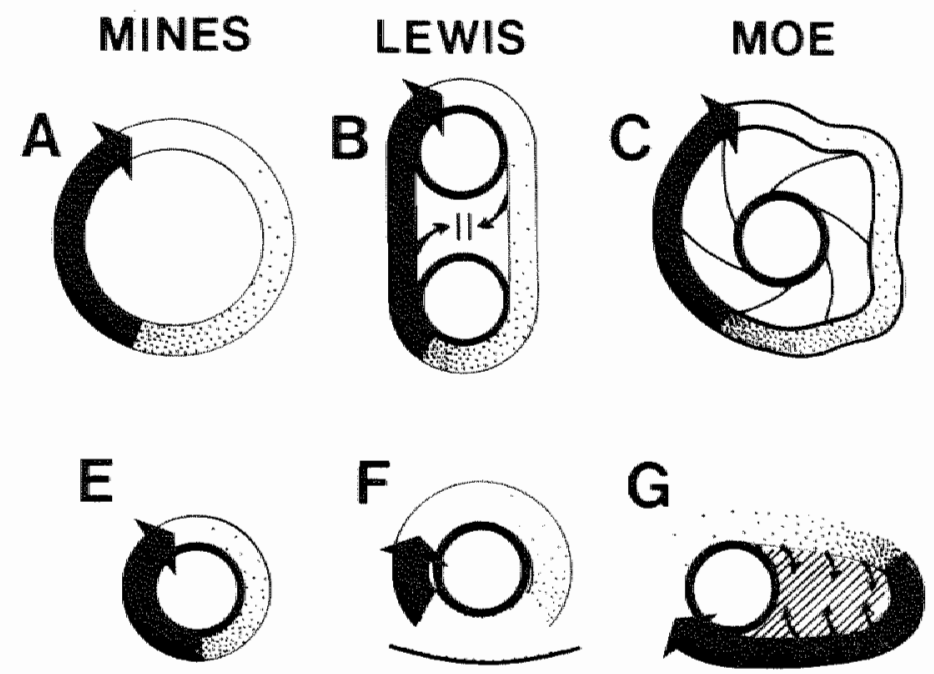

\section{ALLESSIE}
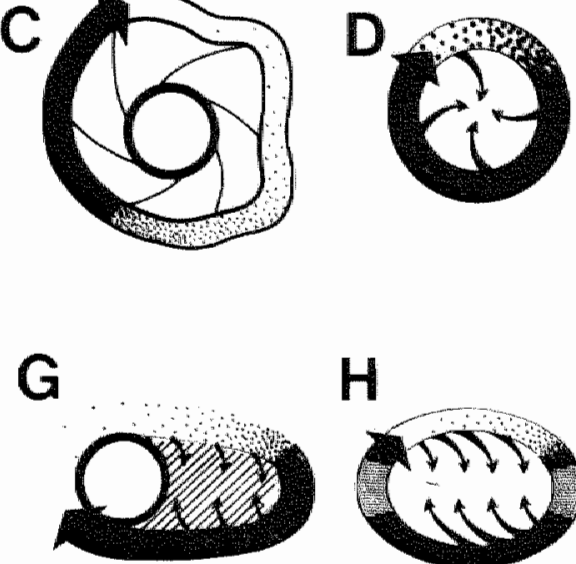

H

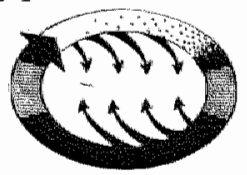

Figure 1.5. Panorama of possible models of circus movements. The black arrows represent the advancing wavefront, followed by the absolute refractory phase. The relative refractory tissue is indicated by the dotted area. The remaining part of the circuit is fully excitable. See text for further description.

created when the impulse propagates slowly at the pivoting points of the central line of block, where it travels perpendicular to the fiber orientation (Lammers et al. 1987).

\subsection{Inhomogeneity in excitability.}

The concept of spatial inhomogeneity in refractoriness, suggested by Mines, was investigated by Lewis (1921) who studied the influence of high stimulation rates on the refractory period: "The shocks fall upon muscle fibres, some of which have recovered, some of which have not; the former respond, and waves of excitation are propagated; the waves are propagated along courses upon which refractory fibres stand; these deflect the waves, prolonging their courses, and thus delay transmission from point to point. The higher the rate of stimulation the more numerous will the 
refractory fibres become: the more numerous the refractory fibres the more sinuous the course of the waves and the greater the delay". Lewis introduced the term "partial refractoriness" to describe this spatial dispersion in refractoriness, but the name did not stick.

Direct demonstration of the spatial distribution in recovery is at the present time not possible; there is no method to visualize and quantify this dispersion. Indirect evidence can be obtained by measuring the refractory period at several sites. Usually, a premature stimulus is applied with a fixed strength and duration but with a variable coupling interval to the preceding regular beat. The shortest coupling interval between the regular and the premature beat which still initiates propagated activation is taken as a measure of the refractory period at the tested site. Alessi et al. (1958) measured refractory periods at different sites in the atrium of anesthetized dogs. Upon stimulation of the vagal nerves, they observed an increase in dispersion of the refractory period from 40 to $100 \mathrm{~ms}$. They also pointed out that induction of an early premature beat at the site with shortest refractory periods often resulted in atrial fibrillation but that fibrillation never followed an early premature activation at sites with longer refractory periods.

Han and Moe (1964) measured refractory periods at twelve sites in the $\operatorname{dog}$ ventricle. As shown in the inset in figure 1.6, the ventricle was stimulated from a central electrode while the recording electrodes were arranged in two concentric rings of six electrodes each, the two rings being located at a distance of 2 and $4 \mathrm{~mm}$ respectively from the stimulus electrode. For the determination of recovery of excitability at the recording sites, each recording electrode in turn was switched from a recording mode to a stimulation mode and the shortest coupling interval between the recorded activity and the induction of a test pulse was measured. Panel A (figure 1.6) shows the pattern of recovery at the twelve test points after a basic beat. The moments of activation of the basic impulse (R1) at the inner electrodes are shown by the solid circles, and for the outer electrodes by the open circles. The moments of earliest possible induction of premature activation (S2) at each recording site are shown by the squares. The R1-S2 interval represents the refractory period at each tested site. During regular drive, there is only a slight dispersion in recovery of excitability, amounting to $6 \mathrm{~ms}$ at the inner points and $12 \mathrm{~ms}$ at the outer points. Series $B$ illustrates the dispersion in recovery following a premature beat. An early premature stimulus (S2) was applied to the central electrode and the recovery time following the 


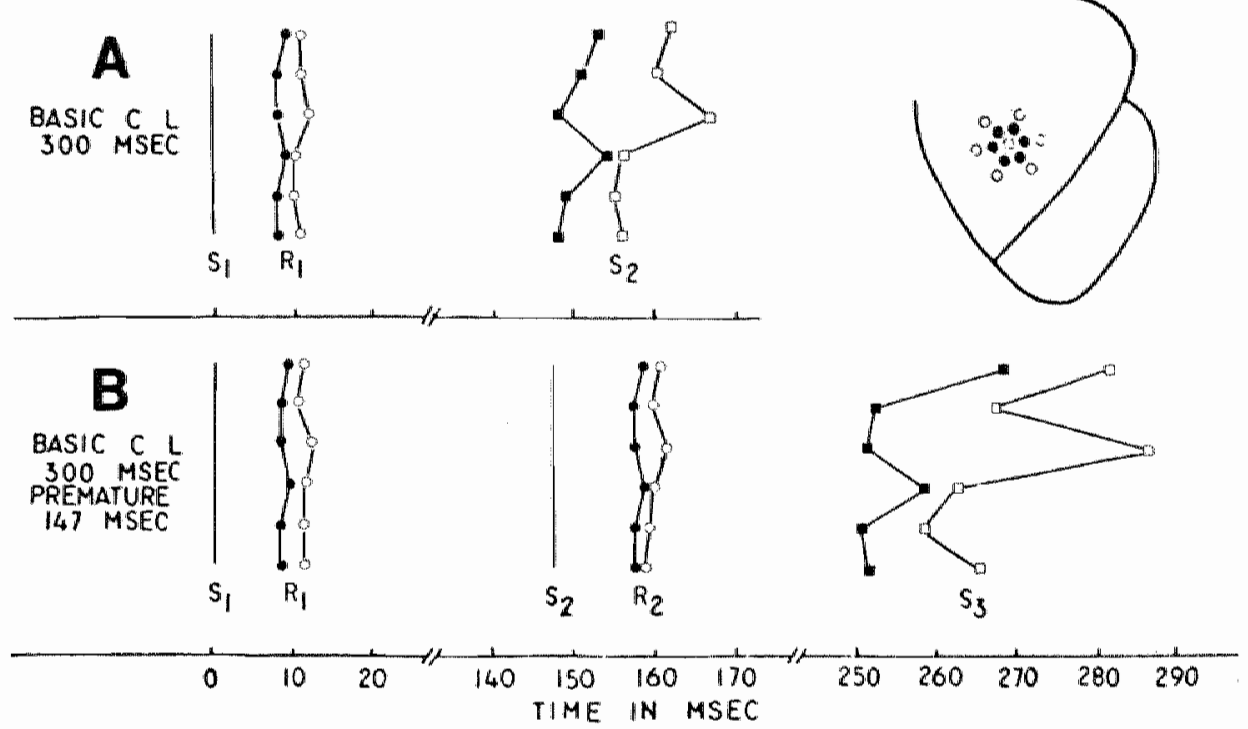

Figure 1.6. Dispersion in recovery following a basic beat (panel A) and after an early premature beat (panel B) as measured in the canine ventricle (Han and Moe 1964). The inset shows the arrangement of the twelve recording electrodes around the central stimulating electrode. In panel $A$, the moments of induction of the earliest possible premature activation at each recording site (S2) represent the local recovery of excitability from the basic activation. In panel $B$, the increased dispersion following an early premature beat is represented by an additional test stimulus (S3). See text for additional details.

premature response (R2) was measured with a third stimulus (S3). It is obvious that the dispersion in recovery was increased following a premature beat (18 and $28 \mathrm{~ms}$ at the inner and at the outer points respectively). In the same study, Han and Moe (1964) investigated the effects of sympathetic stimulation, ouabain, quinidine, ischemia and hypothermia which all increased dispersion in recovery. They conclude: "those agencies known to favor the development of ventricular fibrillation were found to increase the temporal dispersion of recovery of excitability, whether the average refractory period was reduced or increased".

In a subsequent paper, Han et al. (1966a) found that dispersion in 
excitability was higher in atrial tissue compared to the ventricles and that an increase in stimulation rates decreased dispersion of recovery except at the highest pacing frequencies. They further showed a significant correlation between dispersion in refractoriness and inducibility of ventricular fibrillation. In a third paper (Han et al. 1966b), the authors measured dispersion in recovery during early premature activation both close to $(8 \mathrm{~mm})$ and far away $(32 \mathrm{~mm})$ from the stimulus electrode and found maximal dispersion near the site of stimulation.

Following these pioneering studies, a large volume of literature started to accumulate, extending the initial studies by $\mathrm{Han}$ and Moe. For example, Janse et al. (1969) found slight notchings in recordings close to the stimulus site upon early premature stimulation which were absent at more distant terminals and conclude that: "evidently at this distance synchronization of activation occurs, in other words the functional refractory period of the myocardium is in phase". A few years later, Janse (1971) measured dispersion in refractory periods at 12 intramural sites in the canine ventricle. In contrast to the initial work of Han and Moe (1964), he could not find an increase in dispersion of the refractory period after a premature beat. A possible explanation for this discrepancy is the fact that Han and Moe measured refractory periods at 12 sites within 5 minutes whereas Janse allowed the heart to stabilize after each rhythm disturbance. The dispersion in refractory period as measured by Han and Moe may therefore be enhanced by the alternations in rate during their stimulation protocol.

Sarachek et al. (1972) measured nonuniformity of repolarization using suction electrodes in cat ventricles and found a decrease in dispersion upon increase in pacing rate to $240 / \mathrm{min}$ which was followed by an increase in dispersion when the rate was further increased to $270 / \mathrm{min}$. Zipes et al. (1974) found a dispersion in recovery in the atria ranging from 25 to $110 \mathrm{~ms}$. Moe performed computer simulations of fibrillation and demonstrated a higher inducibility of fibrillation upon increase in dispersion of recovery (Moe 1962, Moe et al. 1964). Several studies have manipulated the dispersion in recovery by changing local properties. Naimi et al. (1977) measured refractory periods in normal and in ischemic muscle and found a correlation between dispersion in refractoriness and vulnerability to spontaneous ventricular fibrillation. Burgess and Coyle (1982) also studied dispersion in refractory periods of premature beats in normal and ischemic tissue and found that the dispersion was increased at long coupling intervals but decreased upon reduction of prematurity. Changing local temperatures in the 
ventricles induced large variations in refractory periods together with a higher inducibility for initiating reentrant activity (Wallace et al. 1966, Kuo et al. 1983, Kimura et all. 1986). Downar and Janse observed significant differences in excitability and inhomogeneity in conduction at closely spaced sites within the acute ischemic zone (Downar et al. 1977, Janse and Downar 1977). Dispersion in recovery has also been measured in humans. Wyndham et al. (1977) could induce repetitive atrial discharges upon rapid pacing. At these pacing rates the average refractory period had shortened but dispersion in refractory period was not increased. Simpson et al. (1982) and Buxton et al. (1984) also could not find an increase in dispersion in refractory periods during pacing or premature stimulation in patients with inducible arrhythmias.

A direct comparison between non-uniformity in recovery and reentrant activity was made by Allessie et al. (1976) who measured refractory periods at numerous sites in the isolated left atrium of the rabbit. One of their results is shown in figure 1.7. At the site with the shortest refractory period, a premature impulse was initiated inducing reentrant activity as shown in the activation maps. Conduction block occurred in a region close to the site of stimulation with markedly longer refractory periods. They conclude: "those premature stimuli that initiated tachycardia always were given on the border between two areas with distinctly different refractory periods" and "it was apparent that the premature impulse always propagated in the direction of sites with shorter refractory periods, while it was blocked in the direction in which refractoriness was of greater duration". On the other hand, "in preparations in which there was more or less uniform restoration of excitability, it was difficult or even impossible to find a stimulus site at which tachycardia could be induced". By manipulating local refractory period with carbachol, Allessie and coworkers found that a minimal dispersion of 11-16 ms was sufficient to create conduction block of an early premature beat. Boineau et al. (1980) performed similar mapping studies in the canine atria and could demonstrate "that the circus motion was due to a complex blocking and shaping effect of the nonuniform refractory field".

\subsection{Inhomogeneity in conduction.}

The first report on inhomogeneity in conduction in heart muscle was by Gotch (1910), who showed that, in contrast to the uniform spread of excitation reported by other investigators, the course of 

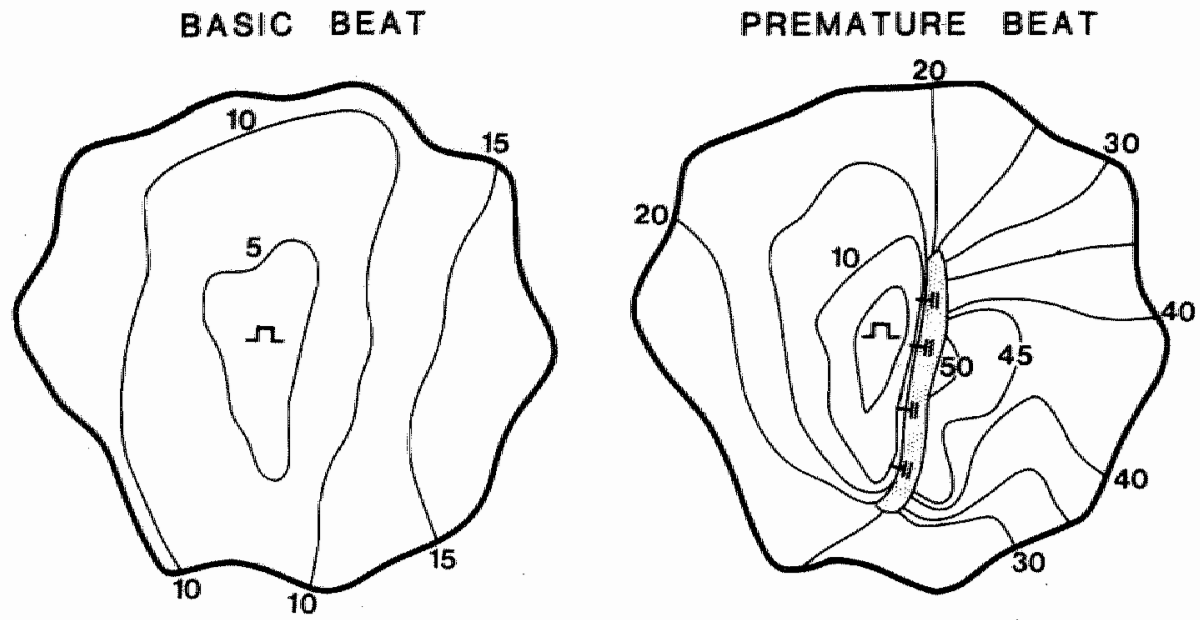

TACHYCARDIA first cycle

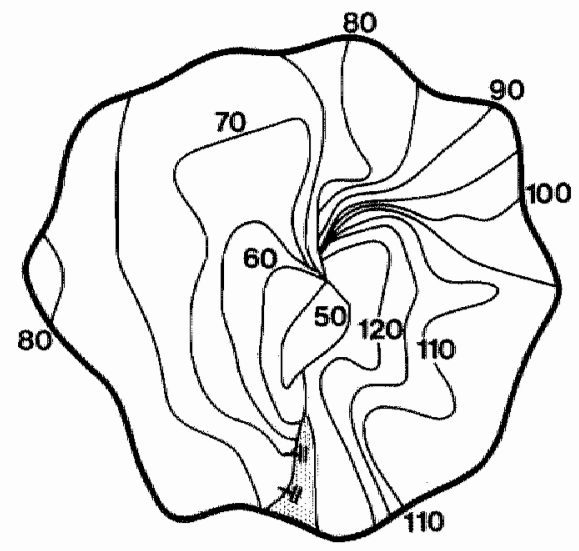

REFRACTORY PERIOD
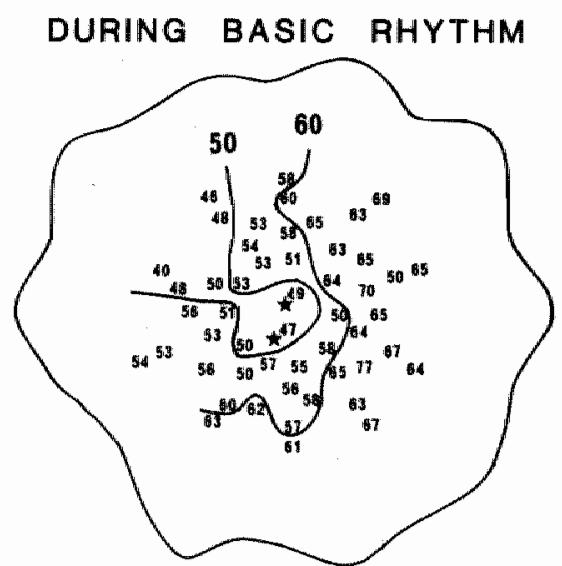

$5 \mathrm{~mm}$

Figure 1.7. Maps of the spread of activation during basic rhythm, an early premature beat and the first cycle of the induced reentrant anthythmia, together with a map of the spatial distribution of refractory periods in the same preparation (Allessie et al. 1976). The premature wavefront was blocked to the right of the site of stimulation (second map), an area which showed markedly prolonged refraciory periods. 
propagated activity in the tortoise ventricle is more complex, following several pathways after initiation at a common site. Lewis et al. (1914, 1915) used the Einthoven string galvanometer to measure the activation times at multiple sites on the dog atria and ventricles. In the canine atria, they showed that conduction was more or less radial in character, originating from the sinus node and spreading out like a "fluid poured upon a flat surface, its edges advancing as an ever widening circle, until the whole surface is covered". In the ventricles however, "... the spread of the excita tory process is far more complex ..." (Lewis et al. 1915) noting that some correlation may exist between the conduction velocity and the orientation of the muscle fibers. Inhomogeneity in conduction was further greatly enhanced if the myocardium was not fully recovered from a previous excitation and in that situation the "crest of the impulse of the wave is flowing through muscle in a partially" refractory state" (Lewis et al. 1921). Drury (1925) studied the effects of pressure on propagation in the canine auricle and concluded: "that the excitation wave travels through compressed" muscle not only much slower than normal, but actually slow down as they penetrate into the damaged region" and "that the successive impulses die out, not because they reach muscle which is irresponsive, but because they have travelled a certain distance through the compressed region". Drury therefore coined the term "decremental conduction" to describe "the gradual failure of conduction as the wave proceeds". Schmitt and Erlanger (1928) used a combination of local pressure and high extracellular potassium to produce local inhomogeneities in conduction. In this situation spontaneous extra systoles occurred. The combination of depressed conduction and extra systoles led them to the formulation of a reentrant mecham nism as shown in the diagram (figure $1.8 \mathrm{left}$ panel). Two groups of fibers (A and B) are unequally compressed thereby creating slower conduction in A (hatched area) than in B. Upon stimulation at 1 the impulse in fibers $A$ is blocked allowing the impulse from fibers $B$ to cross over behind the compressed area and travel back through fibers A. They presented this mechanism to explain the occurrence of spontaneous ventricular extrasystoles in man based "upon the arrangement of the ventricular conduction system" (figure 1.8 right panel).

Later, this model of depressed conduction in the Purkinje system was taken up again by Hoffman, Cranefield and Wit. By encasing part of a Purkinje fiber in high-potassium agar, these investigators decreased local conduction velocities to $5 \mathrm{~cm} / \mathrm{sec}$. Micro-electrode recordings revealed slow conduction and unidirectionall blocks but 

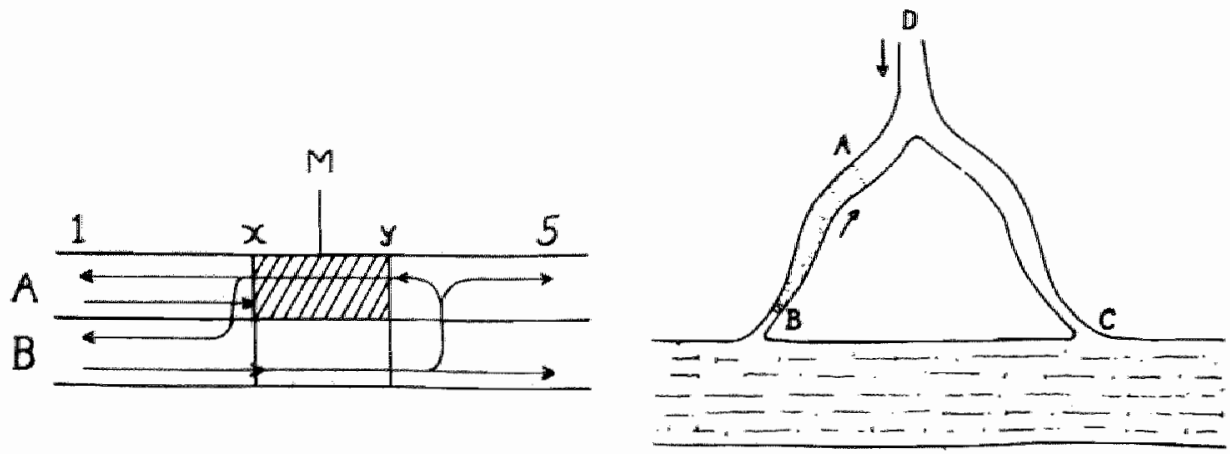

Figure 1.8. Diagram by Schmitt and Erlanger (1929) illustrating their explanation for the initiation of an extra-systole. A unidirectional conduction block was assumed to have occurred in an area of depressed conduction (hatched area) followed by reentry. From this hypothesis, they incorporated the mechanism in the existing Purkinje network of the ventricles (right panel). Segment $A-B$ represents an area of depressed conduction.

also new phenomena such as "summation" and "inhibition" (Cranefield et al. 1971a, 1971b). Summation was shown to occur when two simultaneous wavefronts, entering from different directions into the depressed area, resulted in a propagated impulse, whereas a single wavefront was blocked. But upon a slight modification of the situation, the opposite phenomenon of inhibition could be observed. Upon induction of two wavefronts, no impulse was initiated in the depressed area, whereas a single wavefront could be conducted (for a review on this subject, see the monograph of Cranefield 1975). Wit et al, using the same type of Purkinje preparation as Schmitt and Erlanger, depressed conduction by perfusing with a high-potassium solution containing epinephrine and demonstrated under these conditions the initiation of single reentrant beats (Wit et al. 1972a) and sustained circus movement (Wit et al. 1972b). In every instance, reentrant activity was induced by an impulse which was blocked in one of the Purkinje branches, followed by roundabout conduction along the other fibers and retrograde activation at the initial site of block, as postulated by Schmitt and Erlanger. But whether arrhythmias based on inhomogeneous conduction and reentry in the terminal part of the Purkinje network, can occur in humans is still debatable. Another type of reentry, based upon a 
macro-circuit, encompassing antegrade conduction along one bundle branch and retrograde conduction along the other branch was first demonstrated by Moe et al. (1965). The involvement of such large reentrant loops in certain types of ventricular tachycardia in patients has been suggested by Wellens et al. (1972) and, again, inhomogeneity in conduction and unidirectional block in one of the branches is supposed to set up the reentrant activity. Akhtar et al. (1974) demonstrated such a pattern of excitation but subsequent investigations revealed that this type of reentry is relatively rare (Wellens et al. 1974, Wellens 1975a).

Several factors which may induce unidirectional block have been mentioned in the literature (Cranefield 1975, Janse 1986). Unidirectional block was first shown by Engelmann (1895) who locally applied poisons to the frog sartorius muscle. Lewis postulated that an early premature impulse can be partially blocked by the spatial dispersion of a previous activation. Methods of inducing local depression of conduction velocity by means of pressure, high potassium or ischemia have already been discussed. Singer et al. (1967) have suggested that phase 4 depolarization in Purkinje fibers, enhanced by stretch or hypoxia, may induce unidirectional conduction blocks and reentry. Transient depolarizations, induced by acetylstrophanthidin in Purkinje fibers, were shown to provoke unidirectional conduction blocks at critical pacing frequencies (Saunders et al. 1973). Focal cooling and local crushing (Downar and Waxman 1976) or the application of depolarizing currents (Wennemark et al. 1968) have all been shown to induce inhomogeneity in conduction and unidirectional block. The geometry of the preparation also plays a role. As shown in the elegant computer simulations of Van Capelle and Janse (1976) and in the experiments of De la Fuente et al. (1971), performed in atrial tissue, an impulse propagating from a small strand into a large mass of tissue may be extinguished at the site of insertion. This is due to insufficient efficacy of the small wavefront to bring a large volume of cells to threshold. Retrograde conduction is still possible because the large wavefront would have sufficient "strength" to activate the small strand. Such a situation may also occur at the junctions of the Purkinje fibers and the ventricular myocardium (Mendez et al. 1970, Veenstra et al. 1984, Joyner et al. 1984) and might play a rolle in Wolf-Parkinson-White patients, where a small accessory bundle inserts into the large mass of the ventricles (Neuss et al. 1975, Wellens and Durrer 1975c). Spach et al. (1981, 1982) have pointed to the significance of the geometrical distribution of fibers in relation to unidirectional block and reentry. 
Brendel et al. (1950) made the first attempt to quantify homogeneity in conduction in the canine atria by measuring at multiple sites local conduction velocities with a "compass-electrode" (figure 1.9 panel A). They measured an average conduction velocity of 80 $\mathrm{cm} / \mathrm{sec}$ which varied between 120 and $50 \mathrm{~cm} / \mathrm{sec}$. A plot of the local activation times in relation to the distance from the sinus node from which the impulse originated showed a linear relationship between distance and conduction time (figure 1.9 panel B). This confirmed the postulation of Lewis that activation in the atria during sinus rhythm is essentially radial. The method of plotting activation times versus distance from the site of impulse formation was also used by Sano et al. (1959) in the first demonstration of anisotropic conduction. In ventricular muscle strips of the dog, they
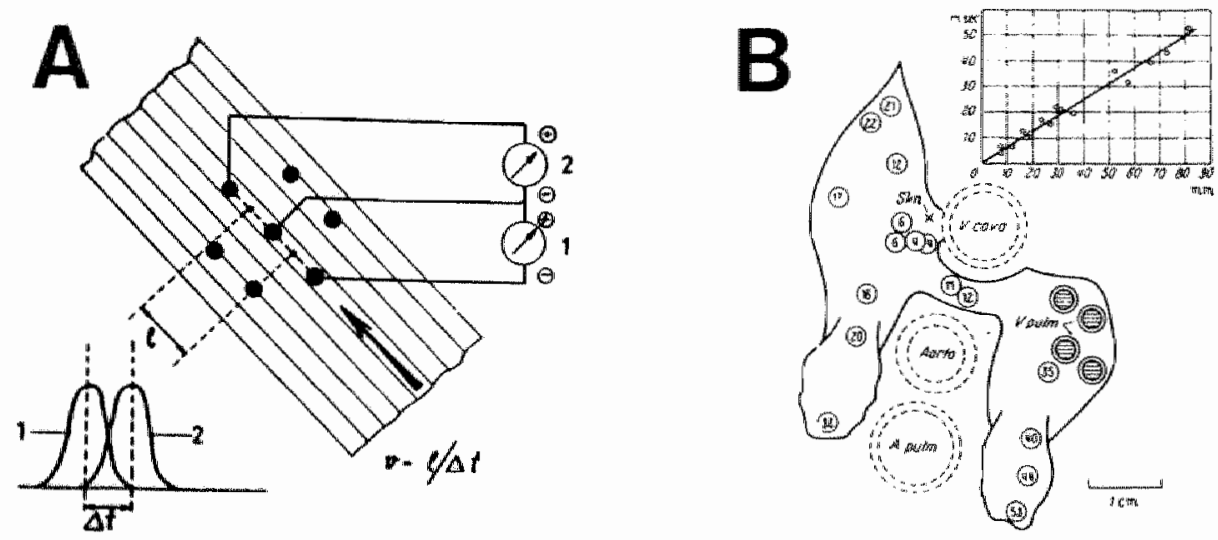

Figure 1.9. Panel $A$ depicts the principle of the compass electrode. Potentials were recorded between $a$ central electrode and two opposite electrodes from the outer ring. Direction of propagation was deduced by the couple that produced a maximal amplitude. Conduction velocity was determined by the time interval between the peaks. In panel $B$, the moments of activation during sinus rhythm, measured in the canine atria, are plotted in the graph in relation to the distance from the sinus node and show a linear relation between activation time and distance. (Skn $=$ sinus node* Brendel et al. 1950) 
observed that conduction parallel to the fiber orientation was two to five times faster than when the impulse was propagating across the fibers. Since then, anisotropic conduction has received considerable attention by several authors (Clerc 1976, Spach et al. 1979, 1981, 1982, 1986a). Myerburg et al. (1973) measured nonuniformity in conduction in Purkinje fibers during early premature activations and concluded that: "this nonuniformity of the wave of excitation became progressively less marked as a function of distance, and at about $10 \mathrm{~mm}$ distal to the site of stimulation, the wave of propagation was almost perfectly uniform across the longitudinal axis".

The coexistence of conduction defects and reentrant arrhythmias in patients has been investigated by several authors. Plumb et al. (1981) found a high incidence of alternans in the electrograms during rapid pacing. In patients with prolonged $P$-wave duration ( $>115$ ms), Simpson et al. (1982) found prolonged intra-atrial conduction times of early premature responses compared to patients with shorter P-wave duration. This observation correlated with a higher incidence of atrial arrhythmias. Similar findings were reported by Buxton et al. (1984).

Since the work of Brendel et al. (1950), the method of plotting local activation times versus the distance from the site of stimulation has been used by several authors (Antoni and Zerweck 1967, Anderson et al. 1970, Shimzu et al. 1979). Although the method has its merits for the measurement of the average conduction velocity, it is not suited for measuring the dispersion in local conduction velocities. In this respect the method is limited by the assumption that propagation occurs in a straight line between the recording electrodes. Any variation in the pathway from this line may induce important variations in the recorded activation times and may lead to erroneous calculations of the true local conduction velocity.

An impressive amount of data concerning conduction properties and possible mechanisms of inhomogeneity in conduction has been gathered, but so far the quantification of homogeneity and inhomogeneity in conduction has been difficult. Therefore, the role of inhomogeneity in conduction could not be compared to other factors known to influence the induction of reentrant arrhythmias, such as dispersion in excitability, wavelength of the impulse or tissue mass. 



\section{Chapter II}

\section{AIM OF THE STUDY}

In an isolated normal superfused left atrium of the rabbit, it is possible under normal conditions to induce intra-atrial reentry with early premature stimuli. Both the location of the stimulus site and the timing of the stimuli are critical for a succesfull initiation of the arrhythmia (Allessie et al. 1973, 1976). Reduction of the potassium content or of the oxygen concentration of the superfusing solution makes the preparation more susceptible to tachyarrhythmias. One objective of this thesis was to study the mechanisms of the tachy-arrhythmias induced in low-potassium or in hypoxia. This was done by visualizing the spread of excitation during the arrhythmias with a multiple recording system (chapter III).

Superfusing with low-potassium is known to enhance abnormal impulse formation (Hiraoka et al. 1979, Eisner et al. 1979), typically showing a rate of $1-3 \mathrm{~Hz}$ (Saito et al. 1978). However, much faster arrhythmias, including fibrillation, have also been demonstrated in low-potassium (Grumbach et al. 1954, Armitage et al. 1957). The mechanism of these tachy-arrhythmias could well be based on the presence of functional circus movements. Mapping the spread of excitation of arrhythmias induced in low-potassium revealed that both abnormal impulse formations and functional circus movements were operative (chapter IV). We aimed to study the factors responsible for the higher inducibility of circus movement in low potassium. An increase in the incidence of reentrant tachy arrhythmias can be caused either by a shortening of the wavelength or by a more frequent occurrence of conduction blocks. As described in chapter IV, the wavelength of early premature beats turned out to be markedly shortened in low-potassium compared to control (14 versus $23 \mathrm{~mm}$ ) whereas no evidence of enhanced inhomogeneity in conduction was obtained.

The mechanisms of arrhythmias induced in hypoxia were also investigated (chapter V). In most cases, functional circus movement was shown to underly these arrhythmias but sometimes abnormal impulse formation occurred as well. The mechanism of these focal arrhythmias is probably early afterdepolarization as shown by 
Trautwein et al. (1954). Again, the objective was to investigate the reasons for the higher incidence of intra-atrial reentry in hypoxia. In contrast to the results obtained in low-potassium, the length of the excitation wave in hypoxia was not greatly reduced (chapter V). Howewer, from the large numbers of activation maps constructed during the induction of the arrhythmias, it could be established that hypoxia enhanced the incidence of unidirectional conduction blocks. Because of the lack of a suitable method to quantify inhomogeneity in conduction, another goal of this study was to develop a method to analyse inhomogeneity of conduction.

First a method was developed to count the number of conduction blocks (chapter VI). With this method, an increase in the number of intra-atrial conduction blocks during early premature activation in hypoxia could be demonstrated. An enhanced inhomogeneity in conduction can be caused either by structural or by functional heterogeneity of the myocardium. Structural conduction properties are, among others, the fiber orientation of the tissue (Clerc 1976), the presence of degenerative changes (Davies et al. 1972) or progressive fibrosis (White et al. 1982). Especially the anisotropic properties of the myocardium, caused by the fiber orientation, has recently received a great deal of attention (Spach et al. 1982, 1986a). Functional inhomogeneities of the myocardium can be caused by the spatial dispersion in excitability, in refractory period (Han et al. 1964, 1966a), in the upstroke of the action potential or in passive electrical properties (Spach et al. 1986b). We investigated whether the spatial dispersion in excitability and in recovery of excitability was enhanced by hypoxia. Such increased dispersion in recovery would enhance inhomogeneity in conduction of an early premature activation, as the premature propagation encroaches on the inhomogeneously retreating tail of refractoriness. Hypoxia turned out to increase both the dispersion in excitability (chapter V) and the spatial dispersion of the refractory period (chapter VI).

A high incidence of conduction block is a sign of increased inhomogeneity in conduction. The method of counting conduction blocks however is not suited to fully describe spatial homogeneity in conduction. We therefore further developed a method to visualize and quantify spatial properties of conduction. This was done by focussing on the local differences in neighboring activation times, expressed as the maximal difference in the local phase of activation. From the activation maps, two-dimensional "phase maps" were constructed which more fully revealed the inhomogeneity in conduction (chapter VII).

The method of phase mapping was used to determine inhomo- 
geneity in conduction in normal superfused preparations and in hypoxic conditions. In hypoxia, inhomogeneity in conduction was shown to be significantly enhanced, both during slow rhythm and during premature propagation. Rapid pacing turned out to induce the largest conduction disturbances. Rapid pacing was also superior to premature stimulation in revealing the location and magnitude of local conduction inhomogeneities, because the results were less dependent on the site of stimulation. Phase mapping can also be used to detect potential sites of reentry, and to describe the size and location of the circuit. 



\section{Chapter III}

\section{METHODS}

\subsection{Experimental Techniques.}

\subsubsection{Preparation.}

New Zealand rabbits of both sexes, weighing between 2 and 2.5 $\mathrm{kg}$, were stunned by a blow in the neck causing cervical dislocation. The chest was opened by a midsternal incision and the heart was rapidly removed by dissecting the connecting veins and arteries and immediately transferred to a glass beaker containing warmed and oxygenated Tyrode solution. The atria were rapidly separated from the ventricles to enable the Tyrode solution to superfuse both endo- and epicardial surfaces of the atria. The time between cervical dislocation and isolation of the atria was less than two minutes. Next a cut was made along the border of the left atrial appendage, removing the base of the appendage (figure 3.1 panel B, hatched area). The left atrium was then dissected from the inter-atrial septum and the orifices of the pulmonary veins were removed. In this manner, a thin sheet of myocardium, consisting of the body of the left atrium and the adjacent appendage, but devoid of any nodal or venous tissue, measuring about 15 by $20 \mathrm{~mm}$, was obtained (figure 3.1 panel $C$ ). The preparation was transferred to a tissue bath and fixed with thin stainless steel hooks on a stretched nylon patch with the endocardial side facing upwards. In some experiments, in which a long narrow strip of atrial tissue was required, the preparation was further dissected by cutting along two parallel lines as indicated in panel C. Usually these strips of atrial myocardium measured 20-30 $\mathrm{mm}$ in length and 2-3 $\mathrm{mm}$ in width.

\subsubsection{Superfusion.}

Rabbit atrial preparations can be kept in normal electrophysiological condition for many hours by superfusing with a solution of the following composition $(\mathrm{mM}): \mathrm{NaCl} 130, \mathrm{KCl} 5.6, \mathrm{CaCl}_{2} 2.2$, 

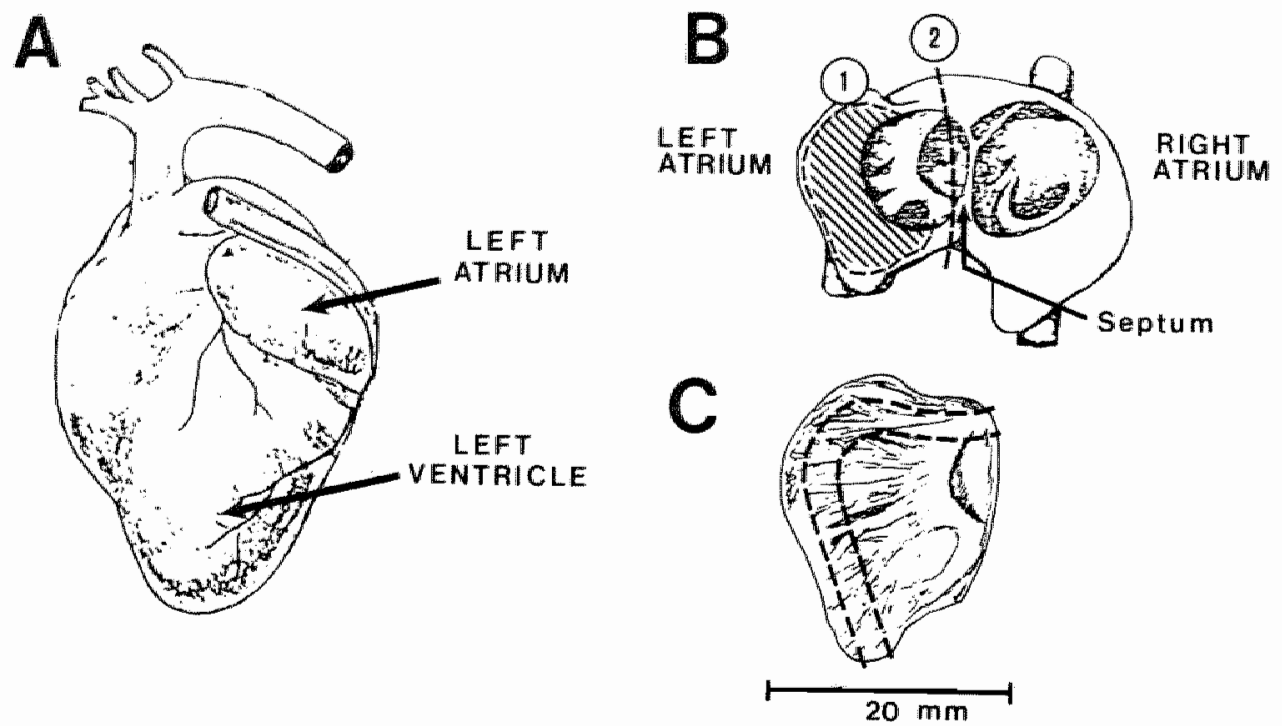

Figure 3.1. Panel $A$ gives a sketch of the rabbit heart as seen from the left. In panel $B$ the atria are separated from the ventricles and turned upside down revealing the two atrioventricular orifices. Two cuts are made (dotted lines), the first to remove the floor of the atrium (hatched area), and the second to separate the left atrium from the inter-atrial septum. After this dissection the isolated left atrial preparation looks as shown in panel $C$ (endocardial side up). The dotted lines indicate the narrow strip which was used in some experiments.

$\mathrm{MgCl}_{2} \quad 0.6, \quad \mathrm{NaHCO}_{3}$ 24.2, $\mathrm{NaH}_{2} \mathrm{PO}_{4}$ 1.2, glucose 11 and sucrose 13. This modified Tyrode solution was prepared on the day of the experiment and stored in 10-liter glass bottles. In these bottles the fluid was kept at $37^{\circ} \mathrm{C}$ by a glass heat exchanger and saturated with carbogen $\left(95 \% \mathrm{O}_{2}\right.$ and $\left.5 \% \quad \mathrm{CO}_{2}\right)$ at a rate of $1-2 \mathrm{l} / \mathrm{min}$. The $\mathrm{pH}$ was $7.4 \pm 0.05$. The Tyrode was pumped to the tissue bath (content 50 $\mathrm{ml}$ ) by a rollerpump (Ismatec) at a flow of $100 \mathrm{ml} / \mathrm{min}$. The fluid oscillations produced by the rollerpump were damped in a glass air-chamber to obtain a continuous flow. Just before entering the tissue bath the Tyrode was led through another heat exchanger to ensure a constant temperature in the tissue bath of $37.0 \pm 0.1^{\circ} \mathrm{C}$. To enable a rapid change of the Tyrode solution, a second supply-route was built in an identical fashion. A stopcock nearby the tissue bath allowed a rapid change from one supply-route to the other. In the low-potassium experiments the potassium concentration was reduced 


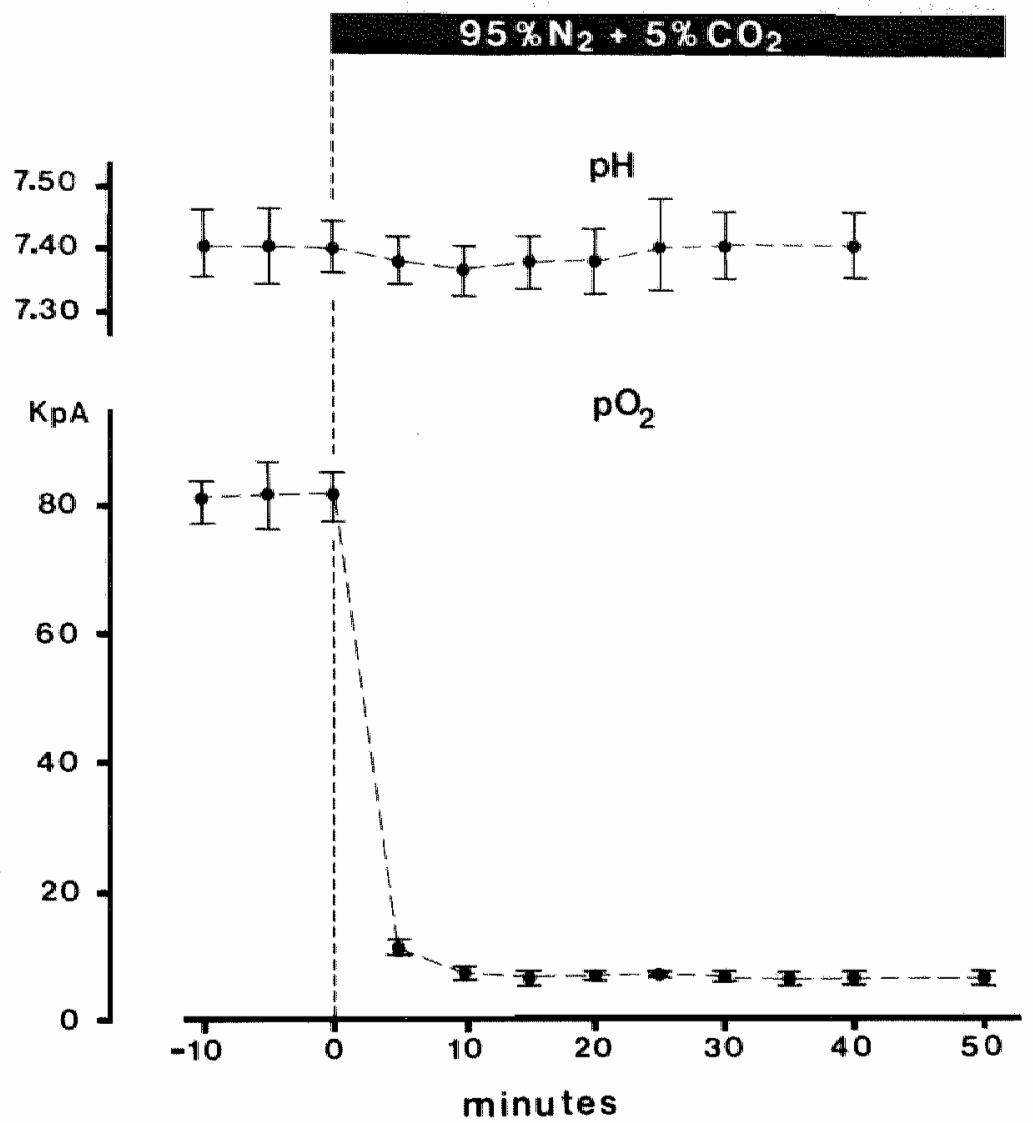

Figure 3.2. Plot of the measured $\mathrm{pO}_{z}$ and $\mathrm{pH}$ in the tissue bath before and after switching from oxygenated Tyrode to Tyrode gassed with nitrogen. The oxygen concentration decreased rapidly to $8 \%$ of its original value. The $p H$ was not affected and remained constant at $7.40 \pm 0.05$.

to $2.0 \mathrm{mM}$. In the hypoxia experiments the test solution was gassed with a 95\% $\mathrm{N}_{2}, 5 \% \quad \mathrm{CO}_{2}$ gas mixture. Figure 3.2 shows the changes in $\mathrm{pO}_{2}$ and $\mathrm{pH}$ when the perfusion was switched from the normal oxygenated Tyrode to the nitrogen saturated Tyrode solution. Samples were taken from the vicinity of the preparation in the tissue bath and immediately analyzed in a $\mathrm{pH} / \mathrm{gas}$ analyzer (Radiometer Copenhagen $\mathrm{ABL}-\mathrm{II})$. The mean $\mathrm{pO}_{2}$ in oxygenated Tyrode 
was $81.5 \pm 3.9 \mathrm{kPa}(611 \pm 29 \mathrm{mmHg})$ while in Tyrode gassed with nitrogen this walue dropped to $6.4 \pm 1.2 \mathrm{kPa}(48 \pm 9 \mathrm{mmHg})$. Switching from the normal oxygenated to the low-oxygen Tyrode reduced the oxygen content of the tissue bath fluid to $8 \%$ of its original value while the $\mathrm{pH}$ remained constant.

\subsubsection{Stimulation.}

The isolated left atrium was stimulated with electrical pulses from a constant-current stimulator between one of the recording electrodes and a large silver plate in the bottom of the tissue bath. Square cathodal pulses with a duration of $1 \mathrm{~ms}$ were used. The threshold for stimulation during diastole normally ranged between 10 and 50 micro-amperes. The preparations were driven at a rate of $2 \mathrm{~Hz}$ with a stimulus strength of two times threshold. When the refractory period was measured or when attempts were made to initiate tachy-arrhythmias premature stimuli with a duration of 1 $\mathrm{ms}$ and a strength of four times threshold were given. The coupling interval between a basic stimulus and the premature stimulus could be varied in steps of $1 \mathrm{~ms}$.

\subsection{Recording Techniques.}

\subsubsection{Microelectrodes.}

Microelectrodes were manufactured with a microelectrode puller (Siegenbeek van Heukelom et al. 1976) which enables reproducible production of microelectrodes. Glass capillaries with an internal glass fiber to facilitate back-filling of the electrodes were used (Tasaki et al. 1968). The microelectrodes were filled with $3 \mathrm{M} \mathrm{KCl}$ and connected with a chlorided silver wire to a high input impedance capacity neutralizing amplifiers (bandwidth $0-13 \mathrm{KHz}$ ) having a fixed gain of 10 . The resistance of the microelectrodes ranged between 5 and 20 MOhm. To minimize $50 \mathrm{~Hz}$ interference, two microelectrodes were used and their outputs, after amplification, were subtracted. One microelectrode was advanced in the cell while the other remained in the extracellular fluid as close as possible to the intracellular electrode.

The resting potential and the amplitude of the action potential were measured on-line using a transient recorder (Datalab DL 901; sampling rate $1000 \mathrm{~Hz}$ ). Samples taken a few milliseconds before the 
upstroke and from the peak of the action potential were continuously plotted on a chart recorder. This method allowed an on-line monitoring of the resting potential and the amplitude of the action potentials with an accuracy of approximately $1 \mathrm{mv}$. To measure the maximum rate of rise, the action potentials were differentiated electronically and continuously displayed on an oscilloscope. The action potentials were also recorded on magnetic tape (Ampex 2200) and action potential durations (at 20\%, 40\%, 60\% and $80 \%$ repolarization) were measured the experiment on an oscilloscope.

\subsubsection{Electrophysiological Mapping.}

\section{- Electrodes.}

Multiple local unipolar electrograms were recorded by an array of electrodes positioned on the atrial preparation. The electrode array (=electrode brush) consisted of 192 teflon-coated silver wires (diameter $0.3 \mathrm{~mm}$ ) which were fixed in epoxy in a regular pattern.

\section{FRONT SIDE}

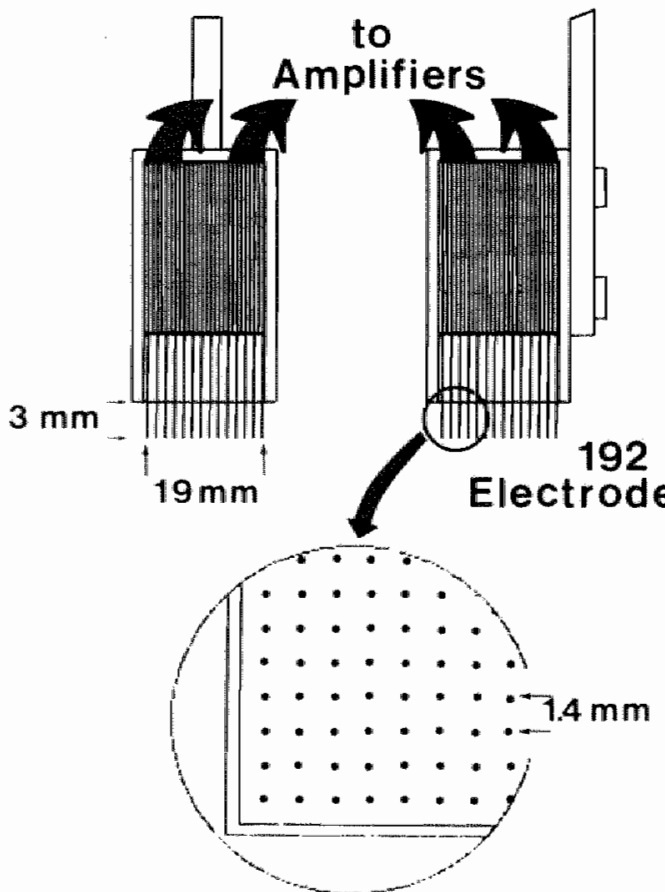

Figure 3.3. A view from the front, side and bottom of a brush electrode. The tefloncoated silver wires (diameter $0.3 \mathrm{~mm}$ ) are glued together in epoxy in a regular array with an interelectrode distance of $1.4 \mathrm{~mm}$. At the top of the brush the silver wires are connected to the positive input of 192 differential amplifiers. At the bottom of the brush the silver wires run free of epoxy for $3 \mathrm{~mm}$ to allow the superfusing Tyrode to reach the covered" surface of the preparation. 
Two different electrode arrays have been used; in the first array the electrodes were positioned in a square of $19-19 \mathrm{~mm}$ and an inter-electrode distance of $1.4 \mathrm{~mm}$ (figure 3.3). The second brush measured 12 by $16 \mathrm{~mm}$ with an interelectrode distance of only 1.0 $\mathrm{mm}$. The electrodes sticked $3 \mathrm{~mm}$ out of the epoxy base to allow the superfusing solution to reach the covered surface of the preparation. The electrode array was mounted on a micro manipulator (Beckmann) with which it was slowly lowered on the preparation while the electrograms were displayed on an oscilloscope (Tektronix 5103N). Care was taken that the pressure of the electrodes on the tissue was not too high and did not provoke injury potentials. After positioning, all electrograms were examined. When the quality of the electrograms was satisfactory, the brush was left in its position during the whole experiment.

\section{- Amplifiers.}

The electrical signals recorded by the electrode brush were connected by 192 coaxial-shielded wires to the positive input of 192 differential amplifiers. A large silver electrode in the bottom of the tissue bath served as a common negative input. Because of the variation in contact of the electrope tips with the surface of the preparation, the amplitude of the recorded electrograms varied. To optimize A-D conversion, individual amplification was performed by an automatic gain system. This feature, which was built in every amplifier, compares the output signal with a pre-set value. When the output signal is larger than 1.4 volt the amplification level of the amplifier "Is automatically decreased until a suitable amplitude is reached. To start the automatic gain setting, a button is pressed to reset all amplifiers at their highest possible gain $(10000 \mathrm{x})$. When the button is released, all amplifiers will reduce their gain individually until an adequate output level has been reached. In practice, only a few seconds are needed for optimal amplification. To prevent accidental re-adjustment of the amplifiers, for example by stimulus artefacts, another switch is provided to lock the automatic gain system.

After adequate amplification, the signals are filtered (bandwidth $2-400 \mathrm{~Hz})$. The value of the low-pass filter $(400 \mathrm{~Hz})$ is related to the sample frequency of the subsequent multiplexer $(2000 \mathrm{~Hz} / \mathrm{chan}$ nel). Filtering of the signal below the speed of the multiplexer is mandatory to prevent aliasing (Cattermole 1969) and good signal approximation is achieved when the multiplexer samples at a rate of five times the highest frequency component of the signal (Steinberg 
ORIGINAL SIGNAL

FILTERED SIGNAL
(Bandwith $2-400 \mathrm{~Hz}$
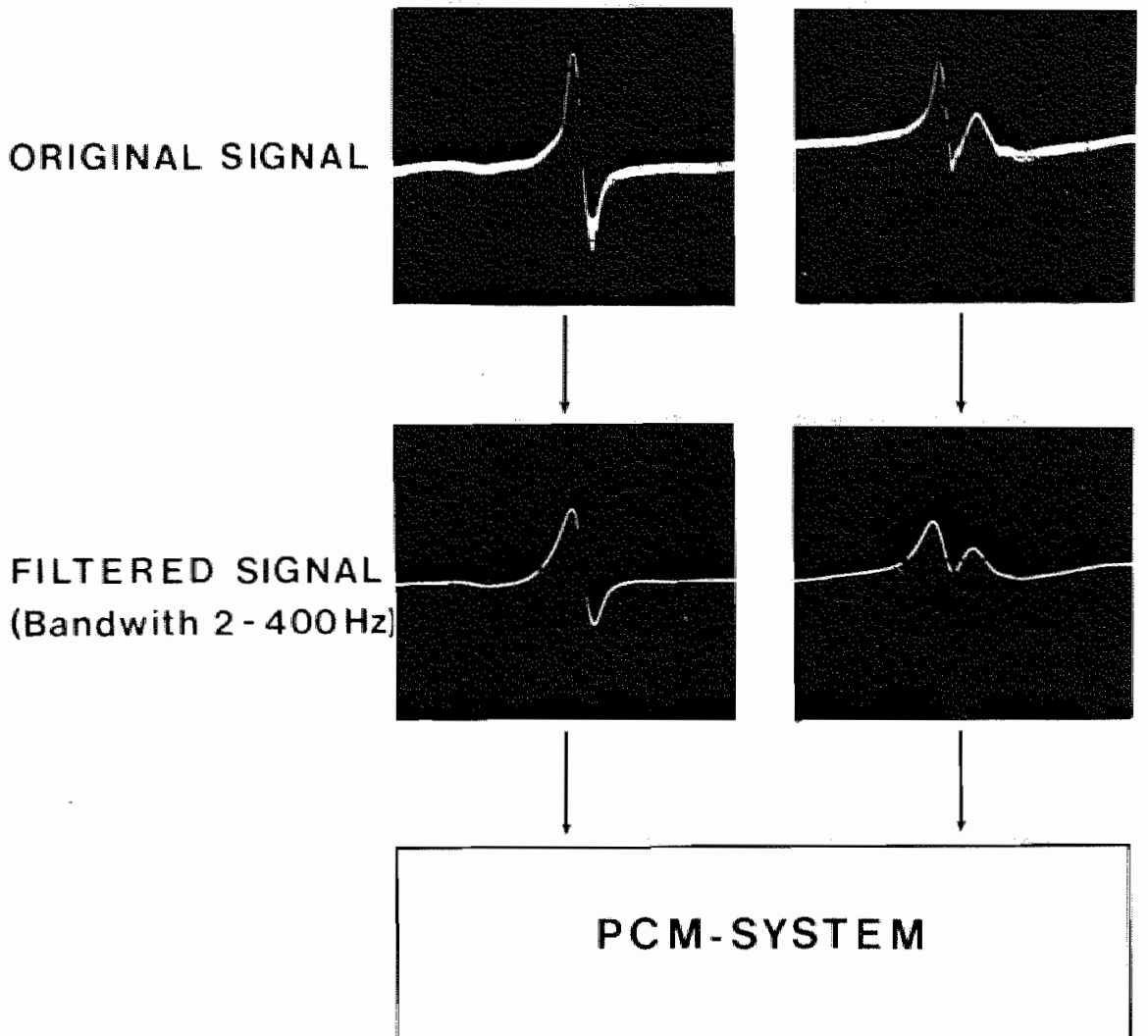

RECONSTRUCTED SIGNAL
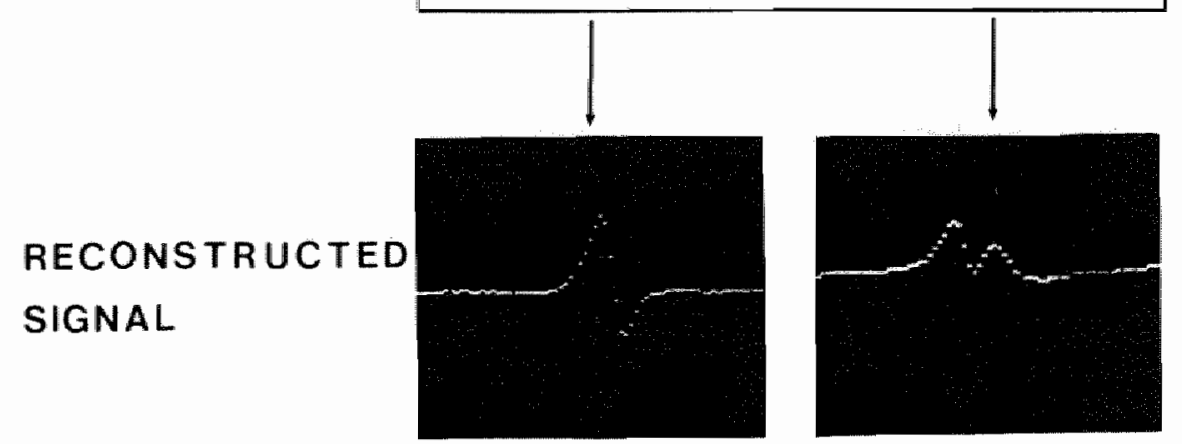

Figure 3.4. Two electrograms recorded from the endocardial surface of a rabbit left atrium preparation (top; bandwidth $0.2-1000 \mathrm{~Hz}$ ). The middle panels show the effect of filtering with a bandwidth of $2-400 \mathrm{~Hz}$ (filtered signal). The lower panels give the sampled signals as played back from the tape (reconstructed signal). 
and Paine 1964, Barr and Spach 1977). Atrial electrograms are not substantially distorted by a cut-off at $400 \mathrm{~Hz}$ and the moment of activation (i.e. the intrinsic deflection) remains easily recognizable after this filtering procedure, as illustrated in figure 3.4 .

\section{- Multiplexers and Analog-Digital Conversion.}

The process of multiplexing and encoding 192 different electrograms is performed in a series of steps (figure 3.5 upper panel). The first step consists of multiplexing all input signals. This is performed by three separate 64-channel multiplexers (Kayser 1280-00) working simultaneously. Each multiplexer samples sequentially the amplitudes of 64 input signals at such a rate that each input signal is sampled every $0.5 \mathrm{~ms}$. The composite signal from each multiplexer, containing the amplitude values from 64 electrograms, is then converted into eight-bit digital words (AD converter in figure 3.5). This digital stream of data is further encoded (Miller code; Mallinson and Miller 1977) and stored on tape.

\section{- Tape Recorder.}

The three high-frequency signals $(0.87 \mathrm{MHz})$ produced by the three encoders, each containing the sampled values from 64 electrograms, are stored on three tracks of a wide-band recorder (Ampex 2230) at a recording speed of 60 IPS (=inch/sec). Because the tape has a length of about 4500 feet, a total uninterrupted recording of 15 minutes can be made. A synchronizing code is added to each encoded signal to achieve an exact timing on all three tracks. During an experiment, additional tracks of the tape are used for the recording of a voice log, a time code (Systron Donner 8350), providing a timing reference for the whole experiment, and one or two reference signals.

\subsection{Data Analysis.}

After the experiment data of interest can be retrieved from tape and analyzed. The off-line analysis starts with the decoding of the previously stored information (Kayser K 1280-10 and $K$ 1240-30) and is in principle the reverse of the encoding system (figure 3.5 lower panel). If the purpose of the off-line analysis is to store and evaluate data in the computer, the digital data is tranferred to disk (Wangco RK05J) allowing the storage of maximally three seconds of 


\section{RECORDING}

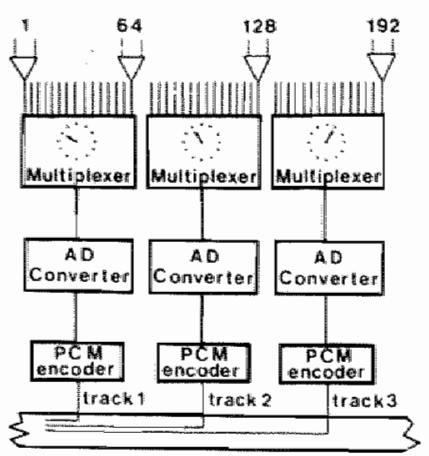

Input-amplifiers

$(2-400 \mathrm{~Hz}\}$

Nultiplexing

(2) Hzicharnes)

AD Convers ion

(11. 3 Mbit/sec)

Pulse Code

Modulation

Tape recordier

(bandwidh $1 \mathrm{MHz}$ )

\section{PLAYBACK}

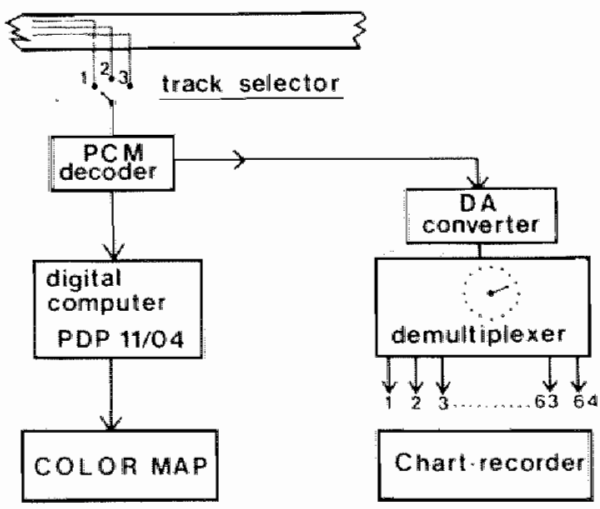

Figure 3.5. In the upper panel a diagram of the multiple recording system is given. All 192 signals are stored on three tracks of the tape. In the lower panel, the off-line data analysis is shown with either a digital computer or reconstruction of the analogue signals for display on a pen recorder. See text for further details.

data. Another possibility is the analogue reconstruction of the input signals by a digital-analogue converter for display on a pen recorder (Schwarzer) or on an oscilloscope (Tektronix 5103N), By means of 16 thumb-wheel selectors maximally 16 signals can be retrieved simultaneously. The data analysis of all 192 signals is performed using a computer (PDP 11-04). The moments of activation in the signals are determined by the intrinsic deflections and stored in a fille. This determination can be done either manually, by plotting the signals on a screen and indicating the intrinsic deflections with a cursor, or automatically, using an algorithm written for this purpose. When all signals have been analysed, the activation times are plotted on a graphic terminal (Tektronix 4010) 
or on a color video monitor (Ramtek 9051) to reconstruct the sequence of activation.

Figure 3.6 illustrates the construction of an activation map. In panel A the locations of 192 electrodes in relation to the borders of a left atrial preparation are given. In this experiment several electrodes had no contact with the preparation but sticked out in the tissue bath fluid due to the small size of the preparation. No electrograms were picked up by these electrodes. The preparation was stimulated through one of the electrodes as indicated by the asterisk (panel B). At the other electrodes the measured activation times in relation to the stimulus moment are displayed. From a few sites, located nearby the stimulus electrode, the electrograms could not be marked due to the presence of a large stimulus artefact while at other sites no accurate marking was possible due to a less optimal contact between the electrode and the surface of the preparation. In this example the signals from 101 of the 109 electrodes $(93 \%)$ located on the preparation could be marked. To visualize the activation sequence, isochrones are drawn manually around areas which are activated within $10 \mathrm{~ms}$. In the activation maps published in this study, the actual activation times are left out for the sake of clarity and only isochrones are displlayed to visualize the sequence of excitation.

\subsection{Measurement of the effective refractory period.}

The preparations were paced at a constant rate of $2 \mathrm{~Hz}(500 \mathrm{~ms}$ interval) with cathodal square pulses with a duration of $1 \mathrm{~ms}$ and an intensity of twice diastolic threshold. After every 20 stimuli a premature stimulus was introduced with an intensity of four times diastolic threshold and a duration of $1 \mathrm{~ms}$. The delay between the last basic stimulus and the premature stimulus (=coupling interval) was increased from a short value, falling well within the effective refractory period, in steps of $2 \mathrm{~ms}$ until a premature activation, as recorded by a nearby recording electrode, was evoked. The shortest possible coupling interval which initiated a premature propagated response was taken as the effective refractory period.

\subsection{Measurement of the conduction velocity.}

In a number of experiments we measured the conduction velocity in atrial strips (figure 3.7). At one end of the preparation a 

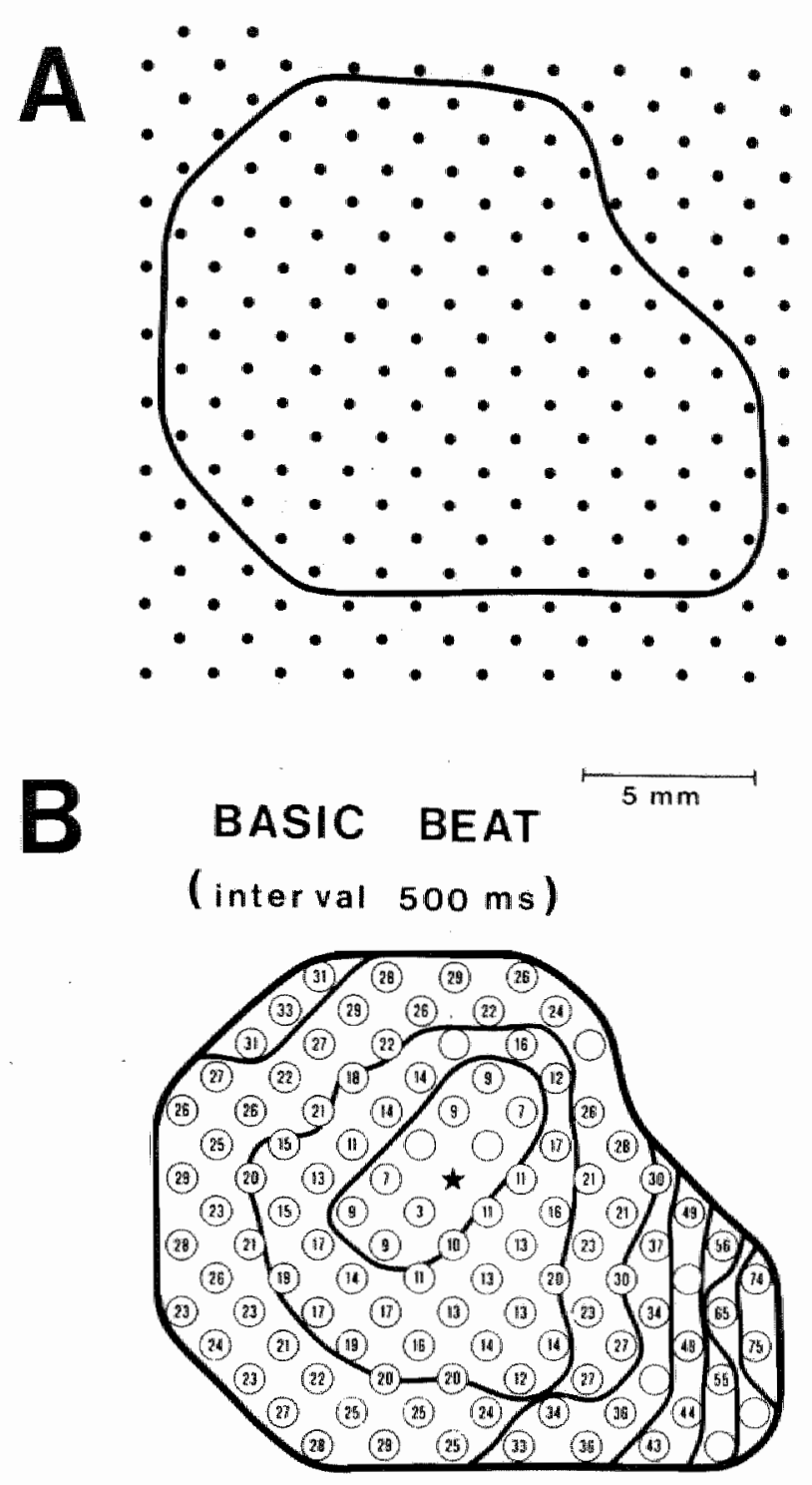

Figure 3.6. Panel $A$ shows the array of 192 extracellular electrodes in relation to the atrial preparation. The inter-electrode distance is $1.4 \mathrm{~mm}$. Panel $B$ gives the reconstructed activation sequence of the atrial preparation during pacing through one of the extracellular electrodes (asterisk). The individual activation times (milliseconds) are related to the stimulus moment and plotted at the respective electrode sites. Isochrones (in time steps of $10 \mathrm{~ms}$ ) are drawn by hand. 

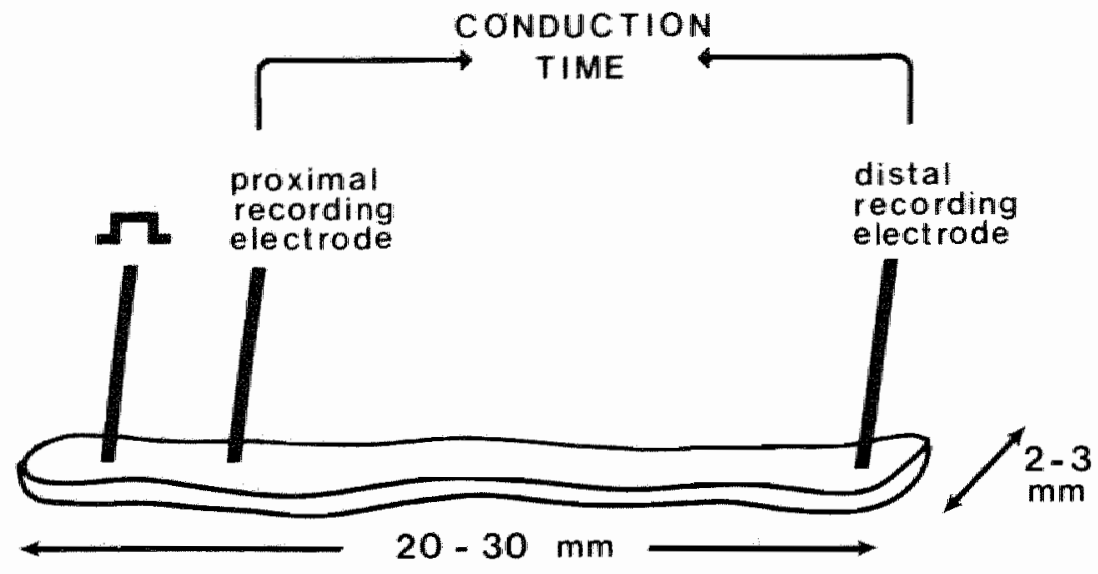

Figure 3.7. Preparation and electrodes used for the measurement of conduction velocity. To force the impulse to propagate in a one-dimensional way, a narrow strip of atrial tissue was used. A stimulating electrode was positioned at one end of the bundle. Conduction time was measured between a proximal and a distal recording electrode, approximately $15-25 \mathrm{~mm}$ apart.

stimulus electrode was positioned. Two recording electrodes were used, one located in the vicinity of the stimulus electrode and the other located at the other end of the preparation. The electrograms recorded from both electrodes were fed into a counter (Hewlet Packard 5308A timer/counter) and the measured time between the fast downstroke ( $=$ intrinsic deflection) of the two unipolar electrograms was plotted on a pen writer (Kipp \& Zonen, BD9). In this manner the conduction time was measured continuously throughout the experiment. 


\section{Chapter IV}

\section{TACHY-ARRHYTHMIAS IN LOW POTASSIUM.}

\subsection{Introduction.}

Hypokalemia is known to facilitate the induction of arrhythmias both clinically and experimentally (Holland et al. 1954, 1958a, 1958b, Kunin e.a. 1962, Curry et al. 1976, Steiness et al. 1976, Surawicz 1980, Nordrehaug et al. 1983). Several studies have investigated low-potassium arrhythmias in isolated atrial preparations of the rabbit using the micro-electrode technique (Saito et al. 1978, Hiraoka et al. 1979, Eisner et al. 1979, Hiraoka et al. 1981). Intracellular recordings during the induction and perpetuation of arrhythmias have provided evidence that mechanisms of abnormal impulse formation play a role in low-potassium. These arrhythmias typically have a rate between 1 and $3 \mathrm{~Hz}$ (Saito et al. 1978). In addition much faster arrhythmias, including fibrillation, can also be induced in low-potassium (Grumbach et al. 1954, Armitage et al. 1957).

The purpose of the present study was:

1. to describe the arrhythmias that can be induced in isolated rabbit atrium in low-potassium;

2. to investigate the nature of the arrhythmias by mapping the spread of excitation;

3. to determine the underlying electrophysiological properties which favor the induction of intra-atrial reentry in low-potassium.

\subsection{Nature of atrial arrhythmias induced in low-potassium.}

In the experiment, displayed in figure 4.1, premature beats were elicited at 9 different sites in an isolated left atrial preparation during control and during superfusion with a low-potassium $\mathrm{mM})$ Tyrode solution. In normal potassium $(5.6 \mathrm{mM})$ no arrhythmias could be induced by application of a single early premature stimulus at any of the tested sites. In low-potassium howewer, bursts of rapid repetitive activity were evoked at eight of the nine tested sites (figure 4.1). Similar results were obtained in seven other 


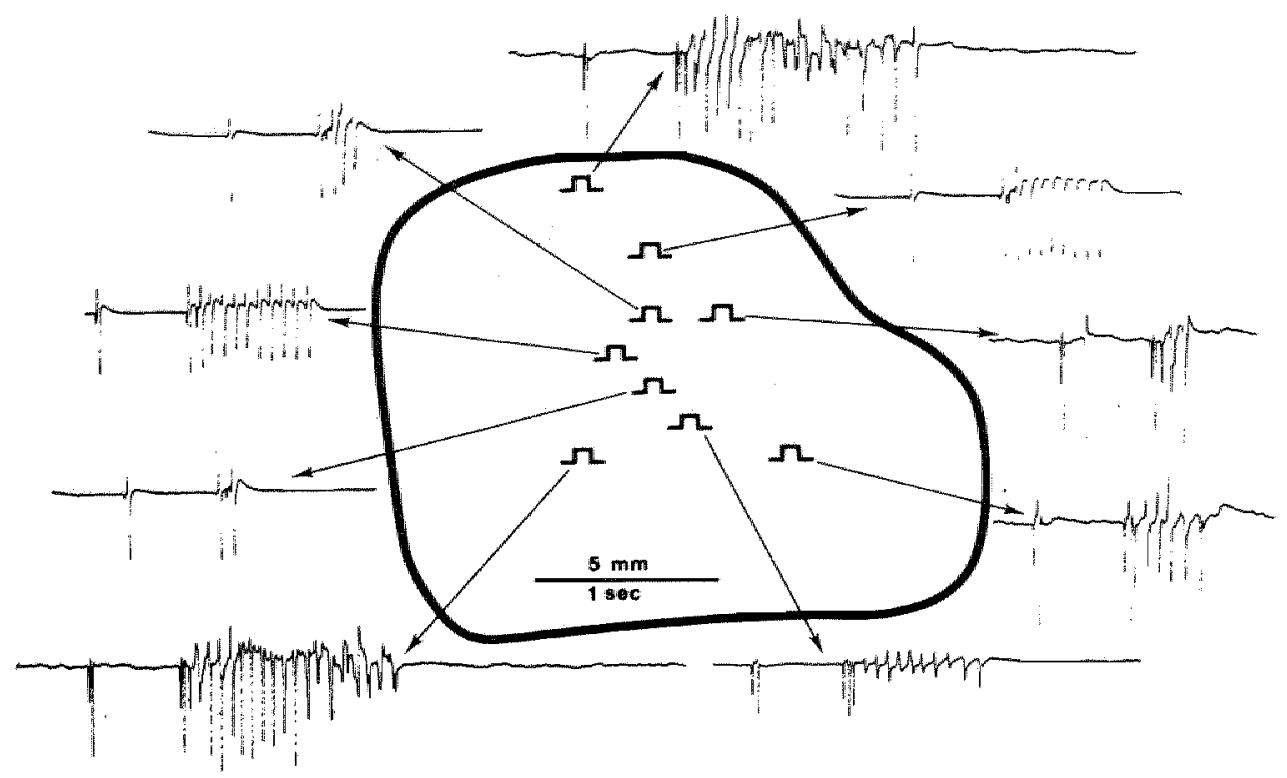

Figure 4.1. The arthythmogenic property of low-potassium was tested by inducing premature beats at several sites in an isolated left atrium of the rabbit. During control $\left(\mathrm{K}^{+}=5.6 \mathrm{mM}\right)$ no arrhythmias could be induced. In low-potassium $(2.0 \mathrm{mM})$ premature stimulation at eight of the nine tested sites induced bouts of rapid repetitive activity, which sometimes degenerated into fibrillation before stopping spontaneously.

experiments demonstrating the arrhythmogenic property of lowpotassium in the isolated left atrium of the rabbit.

Practically all arrhythmias were short-lasting, generally no longer than a few seconds before terminating abruptly. In most cases the arrhythmias had a rate in the range of $10-20 \mathrm{~Hz}$ and sometimes degenerated into fibrillation before terminating spontaneously.

To determine the nature of the arrhythmias the spread of excitation during the initiation of the arrhythmias was mapped in 16 cases. An example is shown in figure 4.2. In the upper panel the stimulus protocol and one electrogram are displayed. The preparation was driven at a basic rate of $2 \mathrm{~Hz}$ for 20 beats (of which the last 9 are shown) and a premature beat was induced with a coupling interval to the last basic beat of $59 \mathrm{~ms}$. In this case the 


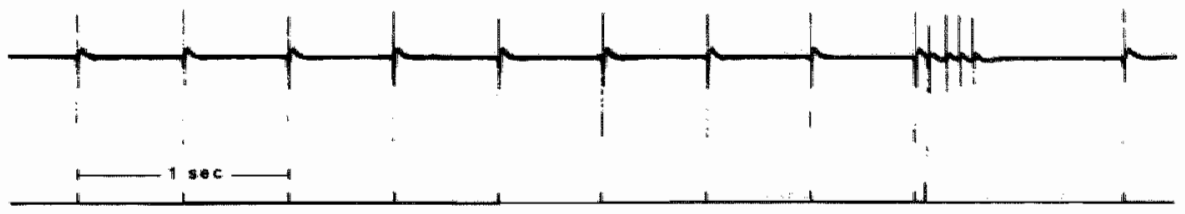

BASIC BEAT

(interwal $500 \mathrm{~ms}$ )

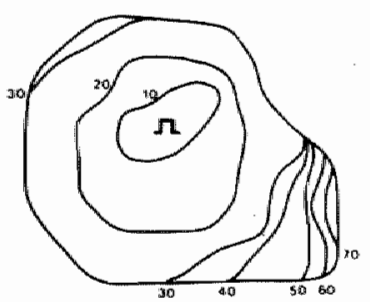

TACHYCARDI A

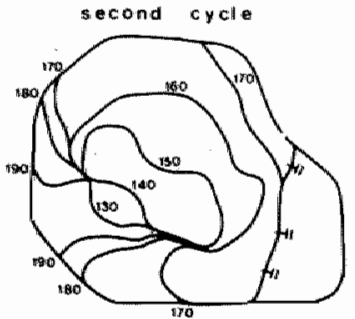

PREMATURE BEAT

(del liay 59 ms )

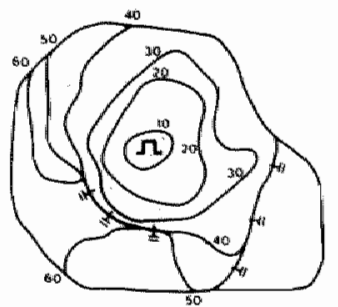

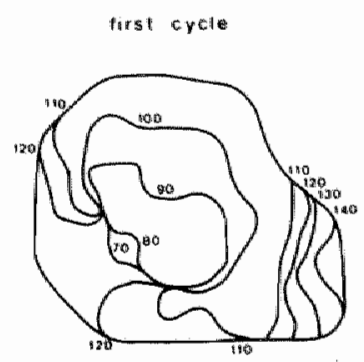

TACHYCARDIA

Figure 4.2. Demonstration of intra-atrial reentry in low-potassium. In the top panel the experimental protocol is shown. The preparation was driven at a basic rate of 2 Hz. After 20 basic beats a premature stimulus (coupling interval $59 \mathrm{~ms}$ ) was given which was followed by four rapid responses. In the lower part of the figure the activation maps of the basic beat, the premature beat and the three spontaneous beats are drawn. During basic rhythm, activation times are related to the moment of basic stimulation while during the premature beat and the subsequent reentrant cycles the activation times are related to the premature stimulus. The premature stimulus initiated a wavefront which can be followed continuously during the whole episode of the arrhythmia. The impulse rotated in both a clockwise and a counter-clockwise direction during the first, second and third reentrant cycle. During the last cycle both reentrant loops were blocked thereby terminating the arrhythmia. 
maps were constructed of the last basic beat, the premature beat and the three spontaneous beats. During basic rhythm $(2 \mathrm{~Hz})$ the preparation was activated radially from the site of stimulation untill the excitation wave was extinguished at the border of the preparation. In the lower-right part of the preparation conduction was markedly slower than in the rest of the preparation as can be seen from the closer isochrone spacing but there were no areas of conduction block during this slow rhythm. The premature impulse (second panel) initially also propagated in a radial fashion although at a slower speed. The slow conduction of the premature impulse is caused by the relative refractory state of the myocardium. At 30 $\mathrm{ms}$ after the stimulus the wavefront was still in the middle of the preparation while during the basic rhythm the wavefront had by that time reached the border of the preparation.

At that moment the premature wavefront encountered an area at the lower left part of the preparation where the impulse was blocked. The arc of conduction block however did not extend to the border of the preparation and the impulse circumvented the area of block, rotating both in a clockwise and a counter-clockwise direction. Both wavefronts collided at the retrograde side of the arc of block, $70 \mathrm{~ms}$ after the initiation of the premature beat.

However the premature wavefront was not extinguished and the area antegrade of the block was reexcited at $80 \mathrm{~ms}$ (third panel). This re-entrant activation was possible because sufficient time had passed for recovery of excitability at that site. From this primary site of reentry the atrium was reexcited and the wavefront continued to rotate in the preparation both in a clockwise and a counter-clockwise direction, initiating a second re-entrant beat at $140 \mathrm{~ms}$ (fourth panel) and a third at $210 \mathrm{~ms}$ (fifth panel). During the third cycle both wavefronts were blocked at $240 \mathrm{~ms}$ and $250 \mathrm{~ms}$ respectively and the arrhythmia was terminated.

As was mentioned above the tachy-arrhythmias sometimes degenerated into fibrillation before terminating spontaneously. Figure 4.3 gives an example. In the upper panel three electrograms recorded simultaneously during a burst of rapid repetitive activity are shown. The electrodes were located at $2 \mathrm{~mm}$ distance from each other. The upper electrogram (A) remained regular during the whole episode of the arrhythmia with respect both to cycle length and to the configuration of the complexes. This is not the case for electrograms $B$ and $C$ which were recorded at 2 and $4 \mathrm{~mm}$ distance from A. During the second half of the arrhythmia these two electrograms became irregular both in shape and in cycle-length. There were sudden variations in configuration ranging from large 

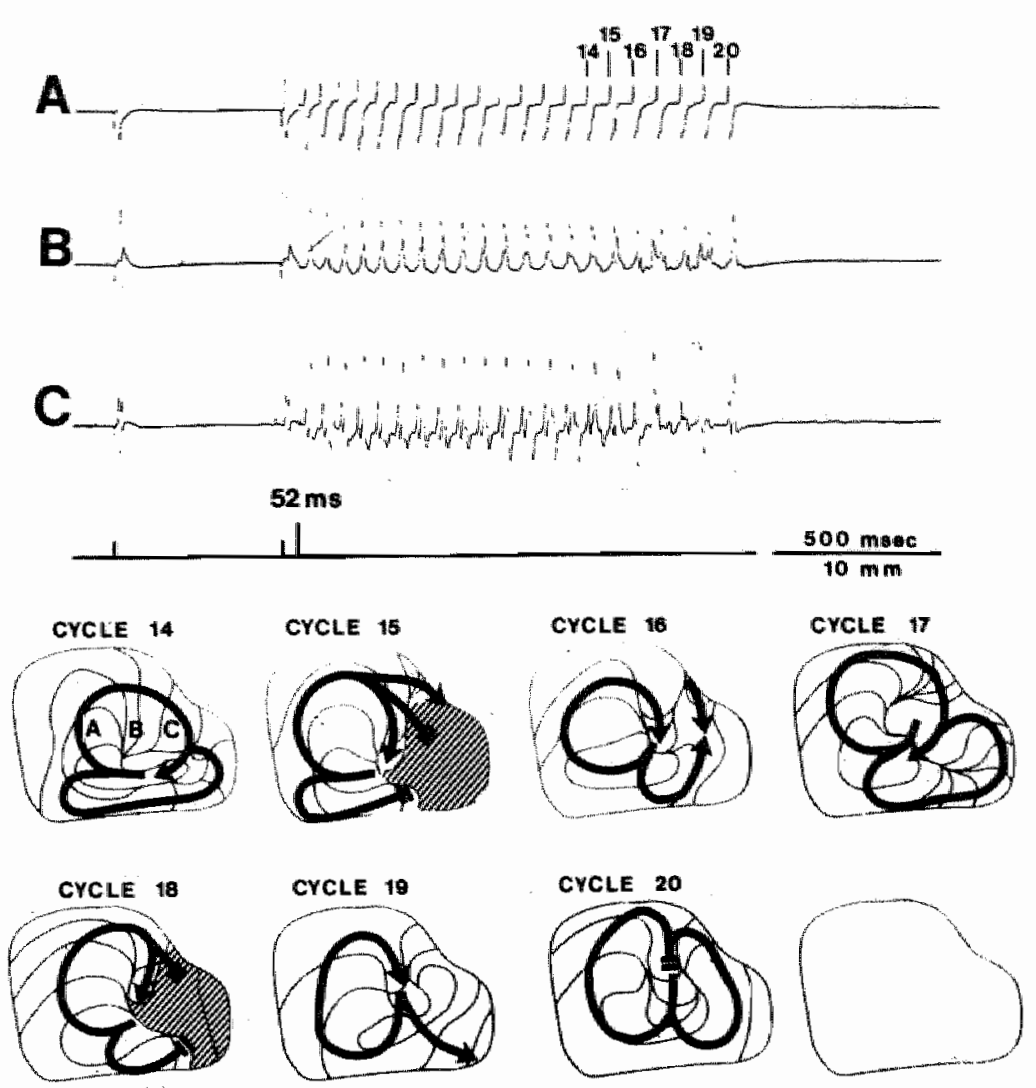

Figure 4.3. The activation sequence of irregular activation during a tachy-arrhythmia in low-potassium. In the upper panel three electrograms are displayed which were recorded simultaneously at adjacent sites as indicated in the first activation map. The 14th cycle was the last regular cycle of the arrhythmia. The activation map reveals two reentrant loops linked together by a common pathway. During the 15 th cycle a large area of block occurred in the right part of the preparation (hatched area) dividing the approaching impulse in two wavelets. One wavelet entered the common pathway to re-activate the left part of the preparation. The second wavelet propagated slowly at the border of the preparation to activate part of the right side of the preparation. From the 15th cycle on there was a continuously changing conduction pattem in the right part of the preparation. This was the basis for the irregularities in intervals and configuration in electrograms $B$ and $C$. Mearwhile the left part continued to be activated in a regular fashion as demonstrated by electrogram $A$. 
deflections to slower low amplitude complexes and multiple component electrograms. Variations in cycle length and the loss of a temporal relationship between the adjoining sites strengthen the "fibrillatory" impression. In the lower part of the figure the activation maps of the last cycles of the arrhythmia, including the transition into this chaotic behavior, are drawn.

The first map depicts the activation sequence during the last regular cycle (no. 14) of the arrhythmia. Two wavefronts rotated in a clockwise and a counter-clockwise direction with both circuits linked by a common pathway. During the subsequent cycles up to the spontaneous termination of the arrhythmia the left part of the preparation continued to be activated in a similar manner. During each cycle the impulse originating from the narrow common pathway at the center of the preparation spreads out to a broad wavefront which propagated regularly to the borders of the preparation. Accordingly all electrograms recorded from the left part of the preparation showed no changes in interval or configuration during the course of the arrhythmia.

In contrast, activation of the right side of the preparation became erratic. During the 15th cycle a large area of conduction block occurred (hatched area) dividing the clockwise rotating impulse in two small wavelets. One wavelet reexcited the left part of the preparation while the second wavelet found an excitable route along the border of the preparation. The conduction of this wavelet was so slow that it collided against a wave originating from the following cycle (no. 16). Because a large part of the preparation had not been activated during the 15th cycle, local excitability was more restored and conduction during cycle no. 16 was faster than usual. This sequence of events explains the irregularity in activation times and configuration of the second and third electrogram in figure 4.3. During the remaining cycles of the arrhythmia (17, 18, 19 and 20) the right part of the preparation continued to be activated in an irregular fashion until in the last cycle conduction was blocked in the common reentrant pathway. In this analysis we did not find any evidence of spontaneous impulse formation. Instead local "fibrillation" in the right part of the preparation occurred due to variable local conduction block, leading to continuous changes in direction and velocity of the propagating wavefront.

In total, 16 low-potassium arrhythmias were mapped. In 12 cases (75\%), the arrhythmia was caused by intra-atrial reentry. The rate of these arrhythmias was in the range of $10-20 \mathrm{~Hz}$.

In $25 \%$ of the analyzed arrhythmias another mechanism was 


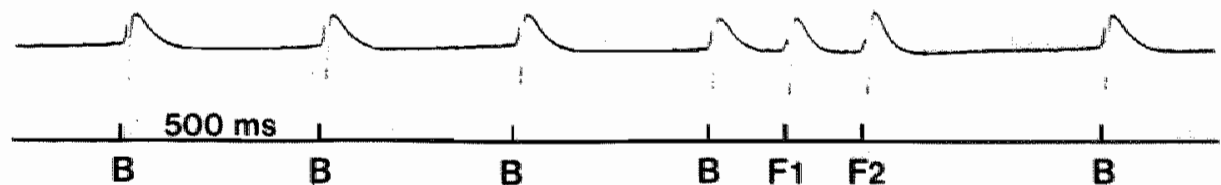

FURST

SECOND
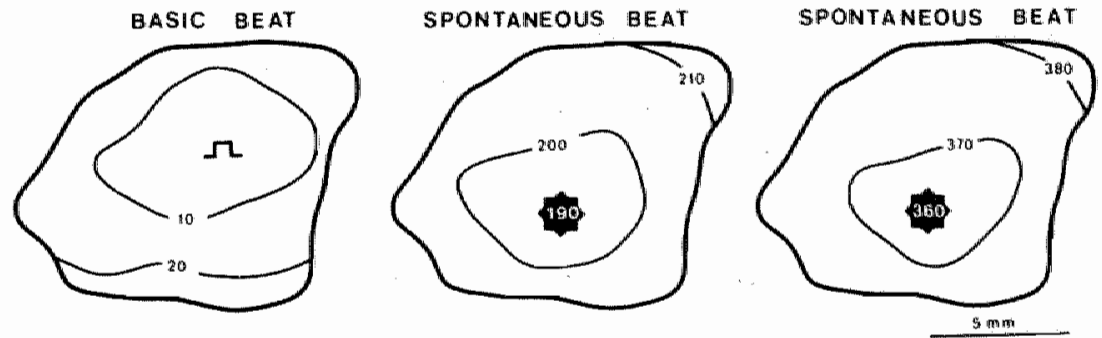

Figure 4.4. Abnormal impulse formation in the rabbit left atrium in low-potassium. During regular pacing (interval $500 \mathrm{~ms}$ ) two successive spontaneous impulses arose at a couple of millimeters from the stimulating electrode. No sign of continuous activity between the stimulus and the first spontaneous beat nor between the two spontaneous activations could be found. There was a clear period of quiescence of about $170 \mathrm{~ms}$ between the stimulated beat and the first spontaneous beat and a second period of quiescence of about $150 \mathrm{~ms}$ between the first and second spontaneous beat.

found, an example being pictured in figure 4.4. The isolated left atrial preparation was again paced at a basic rhythm of $2 \mathrm{~Hz}$ during perfusion with a low-potassium solution $(2,0 \mathrm{mM})$. During this basic rhythm several non-driven beats occurred. The activation of the last basic beat and the first two spontaneous beats were analyzed. During basic stimulation (first panel) the preparation was activated rapidly and radially from the site of stimulation. After extinction of the impulse at the border, the preparation was quiescent until $190 \mathrm{~ms}$ after the basic stimulus. At that moment excitation emerged at a site several millimeters distant from the stimulus site. This spontaneous impulse activated the preparation in a rapid and radial pattern. This is followed, after a new period of quiescence, by a second spontaneous impulse originating from the same site and followed by a similar activation sequence. There was 
no detectable electrical activity between the driven beat and the first spontaneous beat nor between the two spontaneous beats. We therefore conclude that this arrhythmia was based on abnormal impulse formation induced at a site distant from the site of stimulation. Characteristics of these arrhythmias were the rellatively slow rate $(1-6 \mathrm{~Hz})$ and the fact that they arose during basic rhythm in the absence of premature stimuli.

\subsection{Why is it easier to initiate intra-atrial reentry in} low-potassium?

As discussed in chapter $I$, the induction of intra-myocardial reentry is favored by an increase in inhomogeneity in conduction and/or a shortening of the length of the excitation wave (Smeets. et al. 1986). Because the wavelength depends both on the refractory period and the conduction velocity, we investigated how these two electrophysiological properties were changed by decreasing the potassium content of the superfusion fluid.

The refractory period was measured at several sites in the isolated rabbit left atrium by measuring the shortest possible coupling interval at which a single premature stimulus could induce a propagated response. The preparations were driven at a rate of 2 $\mathrm{Hz}$ for 20 beats before a test stimulus was given. Basic and premature stimuli had a duration of $1 \mathrm{~ms}$. The strength of the basic stimulus was twice threshold and the test stimulus was four times threshold. The results from eight experiments are displayed in figure 4.5. During control the refractory period ranged between 63 and $133 \mathrm{~ms}$. During low-potassium $(2.0 \mathrm{mM})$ the refractory period dropped to $44-69 \mathrm{~ms}$. This reduction in refractoriness from a mean of $88 \mathrm{~ms}( \pm 18 \mathrm{~ms})$ to $56 \mathrm{~ms}( \pm 7 \mathrm{~ms})$ was statistically significant $(\mathrm{p}<0.001)$.

Conduction velocity was measured in strips of left atrial tissue (see figure 3.7). Figure 4.6 presents the results of a representative experiment. During a regular rate of $2 \mathrm{~Hz}$ conduction time was measured between two extracellular electrodes (interelectrode distance $12 \mathrm{~mm}$ ). In addition the stimulus threshold and the refractory period at the pacing site were also measured. During low-potassium superfusion, the stimulus threshold gradually increased (from 35 to 50 microAmp), refractory period shortened (from 75 to $55 \mathrm{~ms}$ ) and conduction time increased (from $21 \mathrm{~ms}$ to $24 \mathrm{~ms})$. After 45 minutes, superfusion was changed back to a normal potassium concentration $(5.6 \mathrm{mM})$ and all variables returned 

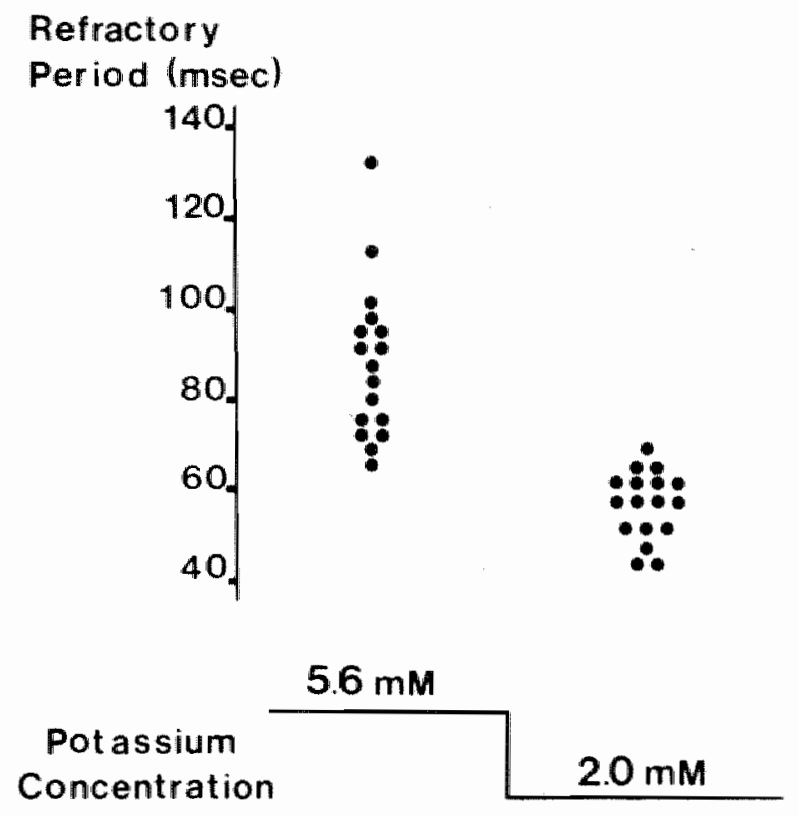

Figure 4.5. The effect of low-potassium on atrial refractoriness. During control $\left(K^{+}=5.6 \mathrm{mM}\right)$ the refractory period was measured at 17 different sites in eight experiments (mean $88 \pm 18 \mathrm{~ms}$ ). Lowering the potassium concentration to $2.0 \mathrm{mM}$ significantly decreased the refractory period to $56 \pm 7 \mathrm{~ms} \quad(p<0.001)$.

to control values within 10 minutes. A second low-potassium trial produced similar results.

From these measurements the changes in wavelength of the cardiac impulse can be calculated (wavelength $=$ refractory period $\mathrm{x}$ conduction velocity). During control a refractory period of $75 \mathrm{~ms}$ and a conduction velocity of $57 \mathrm{~cm} / \mathrm{sec}$ resulted in a wavelength of $43 \mathrm{~mm}$. Lowering the extracellular potassium concentration to $2 \mathrm{mM}$ shortened the refractory period to $55 \mathrm{~ms}(27 \%)$ and depressed conduction velocity to $50 \mathrm{~cm} / \mathrm{sec}(12 \%)$, thus reducing the wavelength from 43 to $28 \mathrm{~mm}$ (35\%).

In figure 4.7 the conduction time of premature impulses is plotted for two different potassium concentrations $(2.0$ and $5.6 \mathrm{mM})$. At 5.6 $\mathrm{mM} \mathrm{K+}$, conduction time was $17 \mathrm{~ms}$ at coupling intervals longer than 120 ms. Shortening of the coupling intervals to values lower than 120 ms progressively depressed conduction until a maximal 

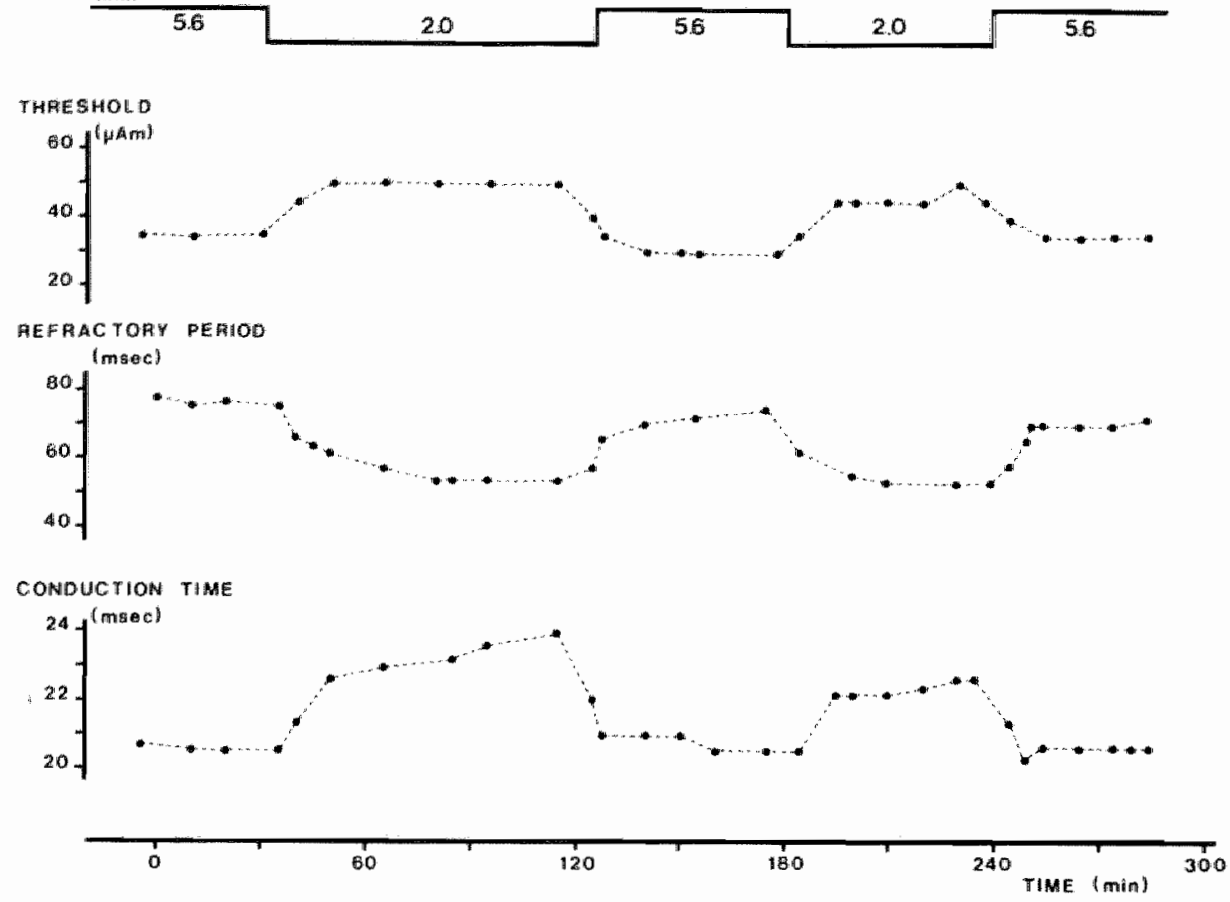

Figure 4.6. The effects of low-potassium on stimulus threshold, refractory period and conduction velocity. Low-potassium induced an increase in the threshold for stimulation, a decrease in refractory period and an increase in conduction time. The effects were completely reversible. A second change to low-potassium produced similar results.

conduction time of 26 ms was reached at the shortest coupled premature impulse.

At low potassium concentration $\left(2.0 \mathrm{mM} \mathrm{K} \mathrm{K}^{+}\right)$the effects of prematurity on conduction were different. At long coupling intervals conduction times were longer than in normal potassium; i.e. conduction velocity was depressed. At coupling intervals shorter than $120 \mathrm{~ms}$, conduction times decreased (conduction velocity increased) with decreasing prematurity up to a coupling interval of $70 \mathrm{~ms}$. Further shortening of the coupling interval produced a gradual increase in conduction time to $28 \mathrm{~ms}$ at the shortest possible coupling interval. In summary low-potassium a) depressed premature conduction initiated at long coupling intervals; b) depressed conduction at the shortest possible coupling intervals and c) increased conduction velocity at intermediate values (supernormal 


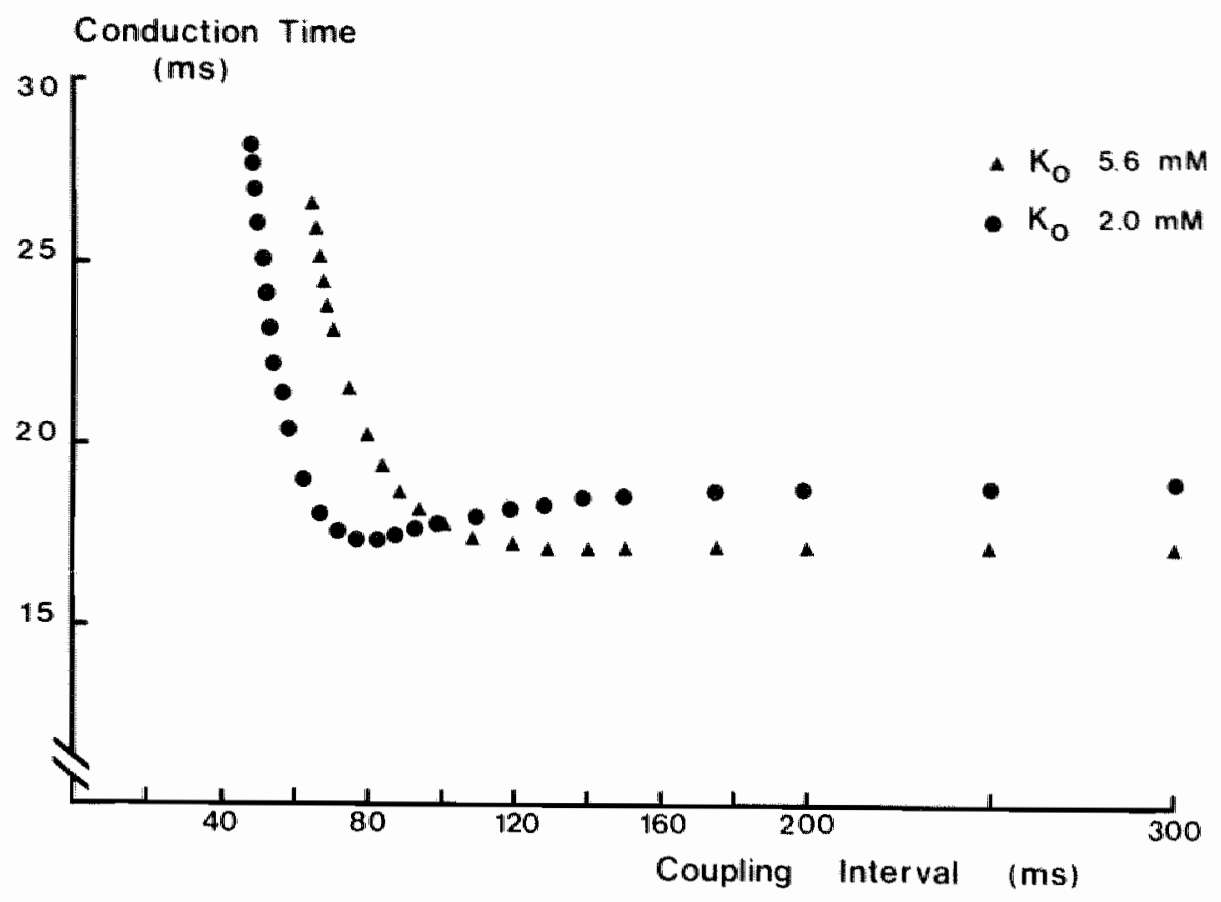

Figure 4.7. The effect of premature activation on conduction time during control $\left(K^{+}=5.6 \mathrm{mM}\right)$ and in low-potassium $(2.0 \mathrm{mM})$. During control (triangles), conduction time was constant at coupling intervals longer than $120 \mathrm{~ms}$. Decrease of the coupling intervals increased gradually conduction time until the highest value was reached at the shortest possible coupling interval. In low-potassium (circles), conduction time first decreased with prematurity (i.e. conduction velocity increased). With further shortening of the coupling interval, conduction time again increased reaching higher values than during control.

conduction, Weidmann 1955). In 7 experiments the magnitude of this supernormal conduction was $7 \% \quad( \pm 3 \% ; \mathrm{p}<0.001)$. The maximum supernormal conduction was reached at an average coupling interval of $91 \mathrm{~ms}( \pm 13 \mathrm{~ms})$. The refractory period in these experiments had shortened to $52 \mathrm{~ms}( \pm 8 \mathrm{~ms})$.

Because premature impulses with a very short coupling interval will most likely induce intra-myocardial reentry, we also measured the wavelength of premature impulses. In three experiments the wavelength was measured during basic rhythm (2 $\mathrm{Hz})$, during the 
earliest premature impulse, and during the fastest possible driving rate. The results are presented in table 4.1. The values found during basic rhythm fit with the data described above; lowpotassium significantly shortened the refractory period and the conduction velocity and therefore the basic wavelength. Lowpotassium also induced a significant shortening of the wavelength of the earliest premature beats $(23 \mathrm{~mm}$ in $4.5 \mathrm{mM}$ versus $14 \mathrm{~mm}$ in $2.0 \mathrm{mM} \mathrm{K} \mathrm{K}^{+}$). This was primarily caused by a shortening of refractoriness. Conduction velocity was not significantly altered. During pacing at the highest possible rate again a significant shortening of the wavelength by low potassium was found (29 $\mathrm{mm}$ versus $24 \mathrm{~mm}$ ) which was completely caused by changes in refractoriness.

\subsection{Dimensions of intra-atrial reentry in low-potassium.}

Except by measuring the wavelength of the impulse in an atrial strip, we could also estimate the length of the excitation wave during low potassium arrhythmias. In figure 4.8 two examples of a run of rapid responses are given together with activation maps

\section{Table 4.1}

The Effects of Low-Potassium on:

\begin{tabular}{|c|c|c|c|c|}
\hline & $\begin{array}{l}\text { Refractory } \\
\text { Period } \\
\text { (ms) }\end{array}$ & $\begin{array}{l}\text { Conduction } \\
\text { Velocity } \\
(\mathrm{cm} / \mathrm{sec})\end{array}$ & $\begin{array}{l}\text { Wave } \\
\text { Length } \\
\text { (mm) }\end{array}$ & \\
\hline Potassium & & & & \\
\hline Concentration (mM) & $4.5 \Rightarrow 2.0$ & $4.5 \rightarrow>2.0$ & $4.5 \cdots>$ & 2.0 \\
\hline $\begin{array}{l}\text { Basic Rhythm } \\
(2 \mathrm{~Hz})\end{array}$ & $\begin{array}{ccc}70 & -> & 50^{*} \\
( \pm 10) & ( \pm 7)\end{array}$ & $\begin{array}{cc}60->> & 52^{*} \\
( \pm 10) & ( \pm 9)\end{array}$ & $\begin{array}{l}42->> \\
( \pm 9)\end{array}$ & $\begin{array}{l}26^{*} \\
( \pm 7)\end{array}$ \\
\hline $\begin{array}{l}\text { Earliest Premature } \\
\text { Beat }\end{array}$ & $\begin{array}{cc}60-> & 39^{*} \\
( \pm 7) & ( \pm 9)\end{array}$ & $\begin{array}{ll}38-> & 34 \\
( \pm 8) & ( \pm 6)\end{array}$ & $\begin{array}{l}23 \cdots> \\
( \pm 6)\end{array}$ & $\begin{array}{l}14^{*} \\
( \pm 5)\end{array}$ \\
\hline $\begin{array}{l}\text { Shortest Pacing } \\
\text { Interval }\end{array}$ & $\begin{array}{ll}78-> & 66^{*} \\
( \pm 8) & ( \pm 13)\end{array}$ & $\begin{array}{l}37->37 \\
( \pm 6) \quad( \pm 6)\end{array}$ & $\begin{array}{l}29--> \\
( \pm 4)\end{array}$ & $\begin{array}{l}24^{*} \\
( \pm 4)\end{array}$ \\
\hline
\end{tabular}



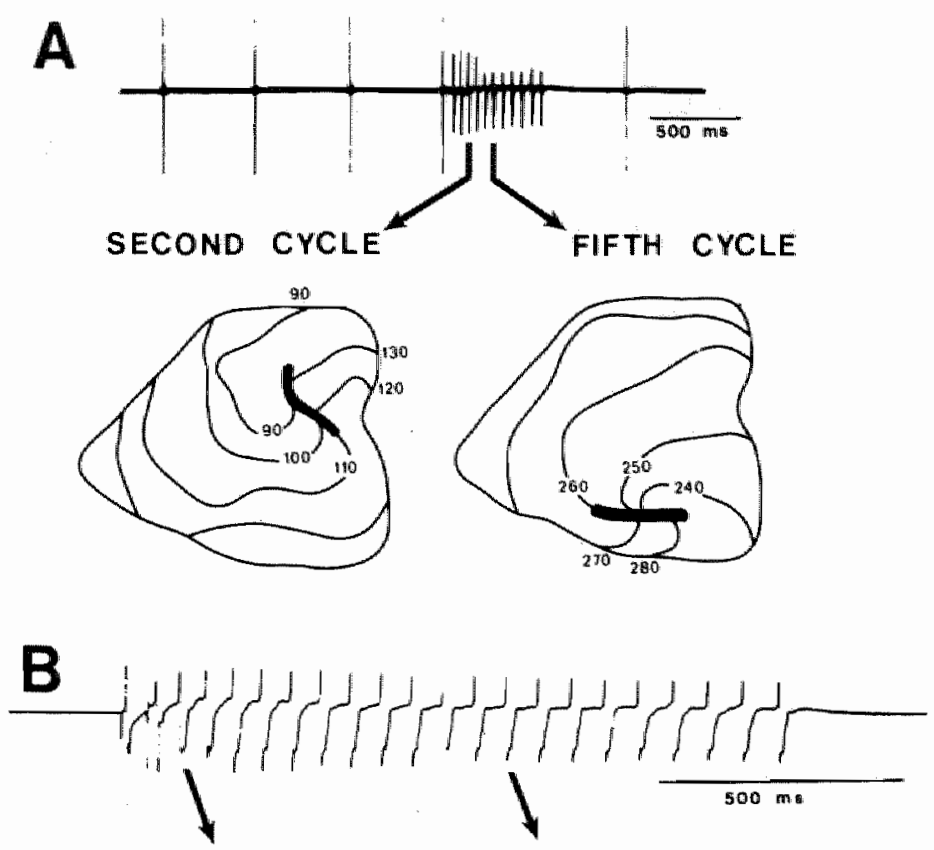

FIRST CYCLE

TWELFTH CYCLE

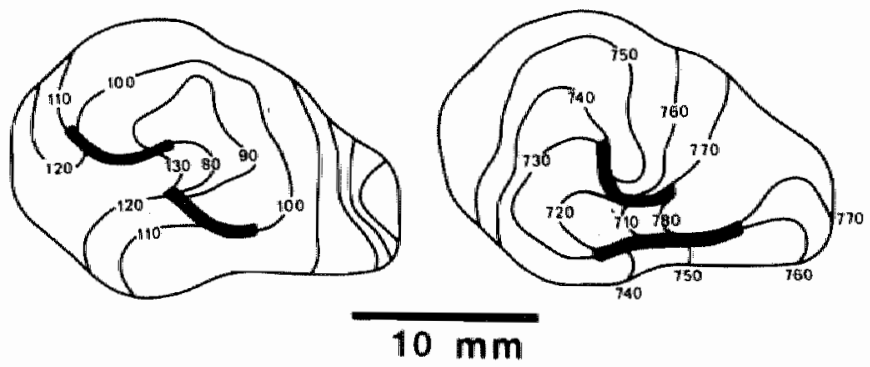

Figure 4.8. Two examples of intra-atrial reenty induced" by a single early premature beat in low potassium $(2.0 \mathrm{~mm})$. In panel $A$ the activation map of the second cycle illustrates a small reentrant loop located in the upper part of the preparation while in the second map (fifth cycle) the circuit rotated in the lower part of the preparation. These shifts in position of reentrant circuits are also demonstrated during another arrhythmia (panel B). In this case two circuits were rotating one in a clockwise and another in a counterclockwise direction. In the twelfth cycle the position of both interlinked circuits had changed markedly compared with the first, again demonstrating the dynamic and functional nature of this kind of intra-atrial reentry. 
elucidating the intra-atrial circuits responsible for the arrhythmias. In panel $A$ the activation maps of the second and the fifth cycle of the first tachy-arrhythmia are reconstructed. During the second cycle the wavefront revolved in the upper part of the preparation while in the fifth cycle the circuit had shifted to the lower part of the preparation. The cycle length in both cases was $50 \mathrm{~ms}$. In panel B two beats (first and twelfth cycle) during another arrhythmia induced in low potassium are depicted. In this case two circuits co-existed one revolving in a clockwise and the other in a counterclockwise direction with a common conduction limb in the center of the preparation. Again the position of the circuits shifted markedly between the first and twelfth cycle. Also the direction of propagation in the common pathway had changed. During the first cycle the common wavefront propagated from left to right while during the twelfth cycle it propagated from right to left. The cycle length had increased from $60 \mathrm{~ms}$ in the first cycle to $80 \mathrm{~ms}$ in the twelfth cycle.

These circuits all rotated around arcs of functional conduction blocks. The length and location of these central arcs of conduction block was determined from the morphology of the extracellular electrograms. As described elsewhere (Allessie et al. 1977, 1984) electrograms recorded on the arcs of conduction block show double complexes. The first part of the complex is caused by activation at one side of the arc and the second part is caused by activation at the other side of the arc. Electrograms recorded distant from arcs of conduction blocks do not demonstrate double complexes.

In figure 4.8 panel A the length of the arcs of conduction block during both cycles was estimated to be 6 millimeters. In the second arrhythmia (panel B) two arcs of conduction blocks were present during each cycle. In the first cycle the length of the arcs was estimated at $7 \mathrm{~mm}$ at the left and $8 \mathrm{~mm}$ at the right. In the twelfth cycle both arcs had increased to a length of $9 \mathrm{~mm}$. The circuit size was estimated to be $12 \mathrm{~mm}$ during the first arrhythmia and 14,16 and $19 \mathrm{~mm}$ respectively during the second arrhythmia.

\subsection{Discussion}

In this study two different kinds of arrhythmias were demonstrated during low-potassium, a rapid rhythm based on intra-atrial reentry and a slower one based on abnormal impulse formation. Saito et al. (1978) obtained evidence for the presence of triggered activity in the rabbit right atrium during low-potassium superfusion 
(2.6 $\mathrm{mM}$ ) using micro-electrode recordings. The dependence of this arrhythmia on the presence of low-potassium was demonstrated by raising the potassium concentration to $5.2 \mathrm{mM}$ which abolished the arrhythmias. The appearance of after-potentials in very low potassium (less than $1 \mathrm{mM}$ ) was also demonstrated in dog ventricular tissue by Hiraoka et al. $(1979,1981)$, in sheep Purkinje fibers by Mugelli et al. (1980), in human papillary muscle by Christe (1982) and in human Purkinje fibers by Dangman et al. (1982).

In our study we confirmed the development of abnormal impulse formation in the atrium at low $\mathrm{K}^{+}$by mapping the sequence of activation. A limitation of this extracellular mapping technique is that the exact nature of impulse formation could not be determined. Indirect evidence however such as the rate of the arrhythmia strongly suggests a mechanism similar to the one described by Saito et al. (1978).

The second type of arrhythmia, based on intra-atrial reentry, has not been described previously in low-potassium. The presence of intra-atrial reentry was established by reconstructing the erratic but circuitous pathway of the impulse during the tachy-arrhythmia. Starting at the moment of premature stimulation we could follow the pathway of the impulse during the arrhythmia until it died out and the arrhythmia was terminated. The position of the intra-atrial circuits was not fixed, but frequently shifted during the course of the arrhythmia. This is not possible if the impulse circulates around an anatomical obstacle. The high rate of the low potassium arrhythmias, together with the absence of areas of block during slow pacing and the continuous shift of the intra-atrial circuit, strongly points to a "leading circle" type of reentry (Allessie et al. 1977 ) around an are of functional conduction block.

We further investigated which electrophysiological properties are responsible for the higher vulnerability to reentrant arrhythmias in low-potassium. The factors which favor intra-myocardial reentry are a) increased inhomogeneity in conduction and b) shortening of the length of the excitation wave. We did not quantify the spatial inhomogeneity in conduction in low-patassium, but focussed our attention on the length of the excitation wave. We found that the wavelength of an early premature beat is shortened by about $40 \%$ when the extracellular potassium concentration is decreased from 4.5 to $2.0 \mathrm{mM}$ (23 $\mathrm{mm}$ versus $14 \mathrm{~mm}$; table 4.1). This shortening is mainly caused by a reduction in refractoriness (from 60 to $39 \mathrm{~ms}$; $35 \%$ ) accompanied by a small decrease of the conduction velocity (from 38 to $34 \mathrm{~cm} / \mathrm{sec}^{2}$ 11\%). A smaller but still significant shortening of the wavelength with $17 \%$ was found during fast pacing. A 
decrease of the refractory period in low-potassium was also measured by Teiger et al. (1967) in isolated rabbit atrium while reduction in conduction velocity was found in papillary muscle and Purkinje fibers (Antoni and Zerweck 1967, Dominguez and Fozzard 1970, Sperelakis et al. 1970).

of special interest is the fact that the wavelength of a single premature beat is smaller than the wavelength during constant rapid pacing (14 versus $24 \mathrm{~mm}$ ). This implicates a gradual prolongation of the wavelength during the initial phase of a reentrant arrhythmia. An increase in the size of the circuits was indeed found in longer lasting tachy-arrhythmias (figure 4.8, panel B).

Finally, the role of supernormal conduction in low-potassium has to be discussed. As was demonstrated in the experiment pictured in figure 4.7, shortening of the coupling interval of a premature impulse resulted in shorter conduction times (i.e. faster conduction) than during basic rhythm. Supernormal conduction has been described in Purkinje tissue by Weidmann (1955) and Spear and Moore (1974a, 1974b) and in Bachmann's bundle by Childers et al. (1968). Supernormal conduction may also play a role in functional circus movement. In the leading circle type of reentry (Allessie et al. 1977) the advancing edge of the wavefront propagates in the retreating tail of refractoriness. If the refractory tissue has just restored its excitability, conduction of the advancing impulse will be slow. But if the impulse is for some reason delayed, conduction in that area may become supernormal. Supernormal conduction may also occur outside the reentrant circuit. An erratic excitation sequence may cause a peripheral wavelet to travel at a cycle length longer than the shortest possible coupling interval. In such an area the activation interval may than fall within the range of supernormal conduction and conduction will be faster. In conclusion the study presented in this chapter has revealed that low potassium increases the vulnerability of the rabbit atrium for intra-myocardial reentry. The easy inducibility of this arrhythmia is probably based on a significant shortening of the wavelength. 


\section{Chapter V \\ TACHY-ARRHYTHMIAS IN HYPOXIA.}

\subsection{Introduction.}

In this chapter the occurrence and the mechanism of tachyarrhythmias initiated under hypoxic conditions are described. There exists consensus in the literature that hypoxia has an arrhythmogenic effect on cardiac tissue (Resnik 1925, Smith et al. 1943, Jacobson et al. 1962). This arrhythmogenic property was easily verified in a few pilot experiments in the isolated rabbit left atrium. As to the mechanisms underlying the tachy-arrhythmias conclusive evidence is not available in the literature. A few reports support the existence of abnormal impulse formation (Trautwein et al. 1954) but circus movement has not been ruled out. If intraatrial reentry occurs during hypoxia, the present studly tries to elucidate the reasons why the impulse is more easily captured in a circus movement.

\subsection{Characteristics of atrial tachy-arrhythmias in hypoxia.}

In 15 experiments the ability to induce arrhythmias in hypoxia was tested by premature impulses applied during a regular pacing rate of $2 \mathrm{~Hz}$. Premature stimuli with short coupling intervals were applied after every 20 basic beats during a control period of 20 minutes and during a period of 60 minutes in hypoxia $\left(\mathrm{pO}_{2}=48 \pm 9\right.$ $\mathrm{mmHg}$ ). In figure 5.1 the inducibility of the arrbythmias during control and in hypoxia is presented. The time scale for the incidence of tachy-arrhythmias is divided in 5-minute periods. During control a tachy-arrhythmia occurred only twice in fifteen experiments. During hypoxia the incidence increased dramatically reaching a level of about $50 \%$ in each time period of 5 minutes. The likelihood of inducing tachy-arrhythmias did not change substantially during the 60-minute hypoxic period.

All these tachy-arrhythmias were initiated by a single premature impulse. Spontaneously occurring arrhythmias were not observed. In four experiments the coupling interval of the premature impulse 
INCIDENCE

EXPERIMENTS

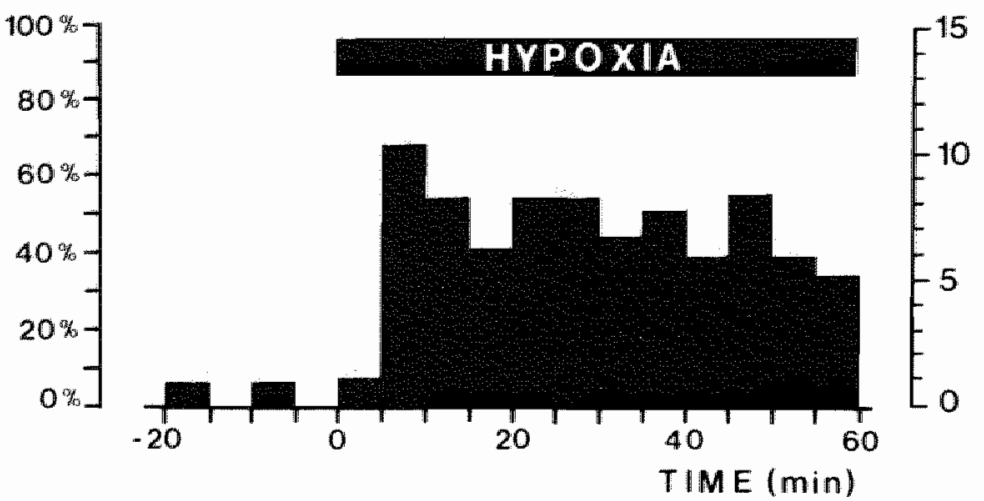

Figure 5.1. Incidence of tachy-arthythmias in hypoxia in 15 experiments. Each bar depicts the number of experiments in which at least one tachy-arhythmia was initiated during a 5-minute time period. The absolute values are given on the right ordinate, the relative incidence on the left axis. The incidence of tachyarrhythmias increased markedly during hypoxia.

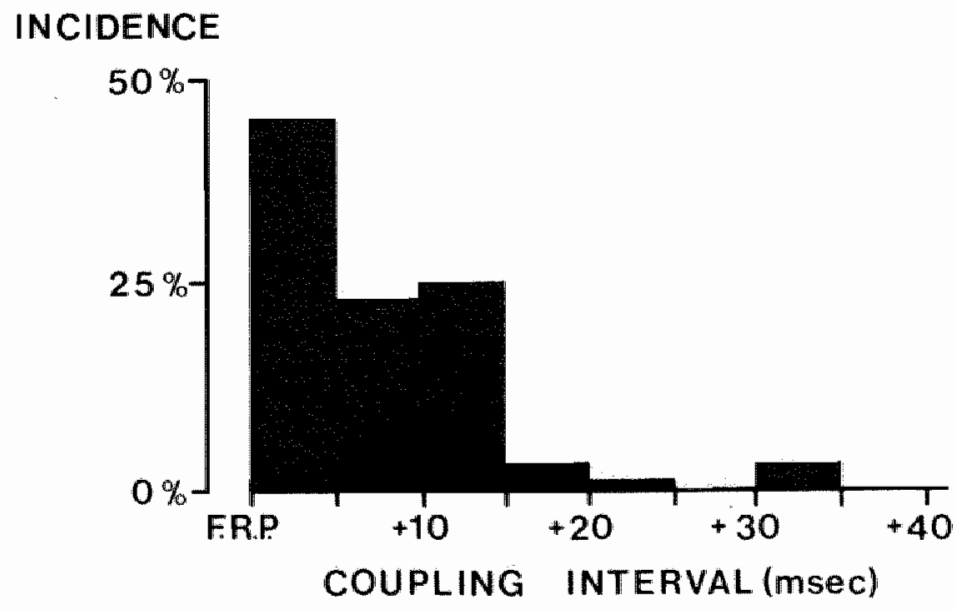

Figure 5.2. The influence of prematurity of an extra stimulus on the initiation of tachy-arrhythmias in hypoxia. The coupling intervals of premature beats that initiated an arrhythmia are plotted on the abscissa in relation to the functional refractory period. The coupling intervals from 73 inductions of tachyarrhythmias are used in this figure. Ninety percent of the tachyarmythmias were initiated by a premature beat elicited within 15 milliseconds after the local refractory period. 


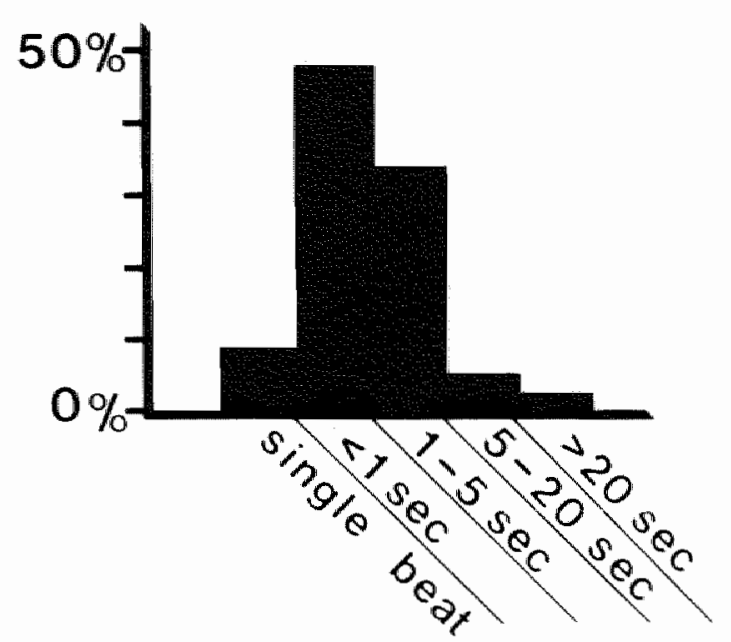

Figure 5.3. Duration of the tachy-arthythmias in hypoxia. Data of all arrhythmias induced by a single premature stimuli in 5 experiments were used $(n=244)$. The majority of arrhythmias (93\%) lasted less than 5 seconds. In $9 \%$ of the cases a single premature beat was evoked. Only a few tachy-arhythmias lasted for periods longer than 20 seconds.

which induced tachy-arrhythmias $(n=73)$ was measured together with the functional refractory period at the stimulus sites (figure 5.2). Ninety percent of the arrhythmias were initiated by premature stimuli within a zone of $15 \mathrm{~ms}$ following the local functional refractory period. The mean coupling interval of the premature beats that induced tachy-arrhythmias was $66 \mathrm{~ms}$ ( $\pm 23 \mathrm{~ms}$ ).

Tachy-arrhythmias initiated in hypoxia tend to stop spontaneously. In five experiments the duration of all induced arrhythmias $(\mathbf{n}=244)$ was measured (figure 5.3). Most arrhythmias (93\%) did not last longer than 5 seconds. Single extra beats only occurred in $9 \%$ of the cases. In figure 5.4 a sequential plot of the beat-to-beat interval of two arrhythmias is presented. In both cases there was a gradual and regular increase in cycle length starting immediately after the initiation of the arrhythmia. There was a slight tendency for this increase to diminish during the arrhythmia but a stable value was not reached due to spontaneous termination of the arrhythmias. Because the tachy-arrhythmias did not become stable we measured the intervals at two different moments during the arrhythmia; the interval after the tenth spontaneous beat was 


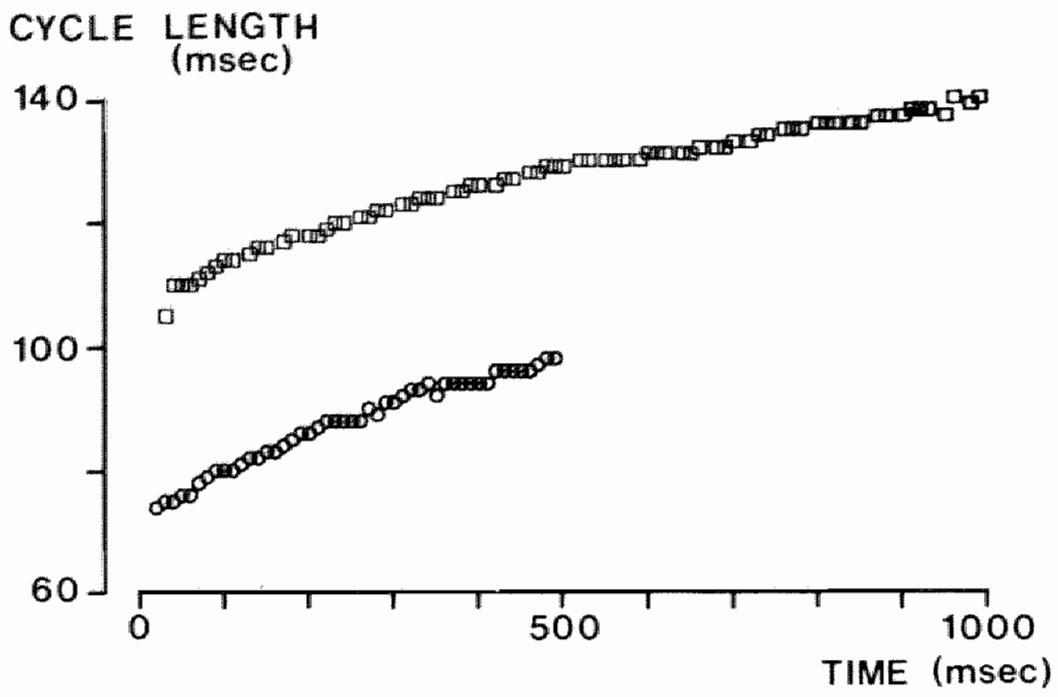

Figure 5.4. Sequential plot of the beat-to-beat interval of two tachy-arrhythmias initiated during hypoxia in the same preparation. In both cases there was a gradual increase in cycle-length until the arrhythmias stopped spontaneously.

$107 \pm 40 \mathrm{~ms}(\mathrm{n}=43)$ and the interval after 2 seconds duration was $124 \pm 33$ ms $(\mathrm{n}=28)$.

In summary, tachy-arrhythmias could easily be induced in the rabbit left atrium during hypoxia by a single premature beat with a coupling interval within $15 \mathrm{~ms}$ after the functional refractory period. The tachy-arrhythmias displayed a gradual increase in cycle-length and tended to stop spontaneously within a few seconds.

\section{Mechanisms of the tachy-arrhythmias.}

The underlying mechanism of the tachy-arrhythmias induced in hypoxia was studied with the multiple recording system described in chapter III. In figure 5.5 a tachy-arrhythmia was initiated by a premature stimulus with a coupling interval of $87 \mathrm{~ms}$ (upper panel). Activation maps were constructed of the last basic beat, the premature activation, the first two spontaneous activations and the last two activations before spontaneous termination. The stimulus electrode was located close to the left border of the preparation as shown by the stimulus symbol in the basic and the premature 

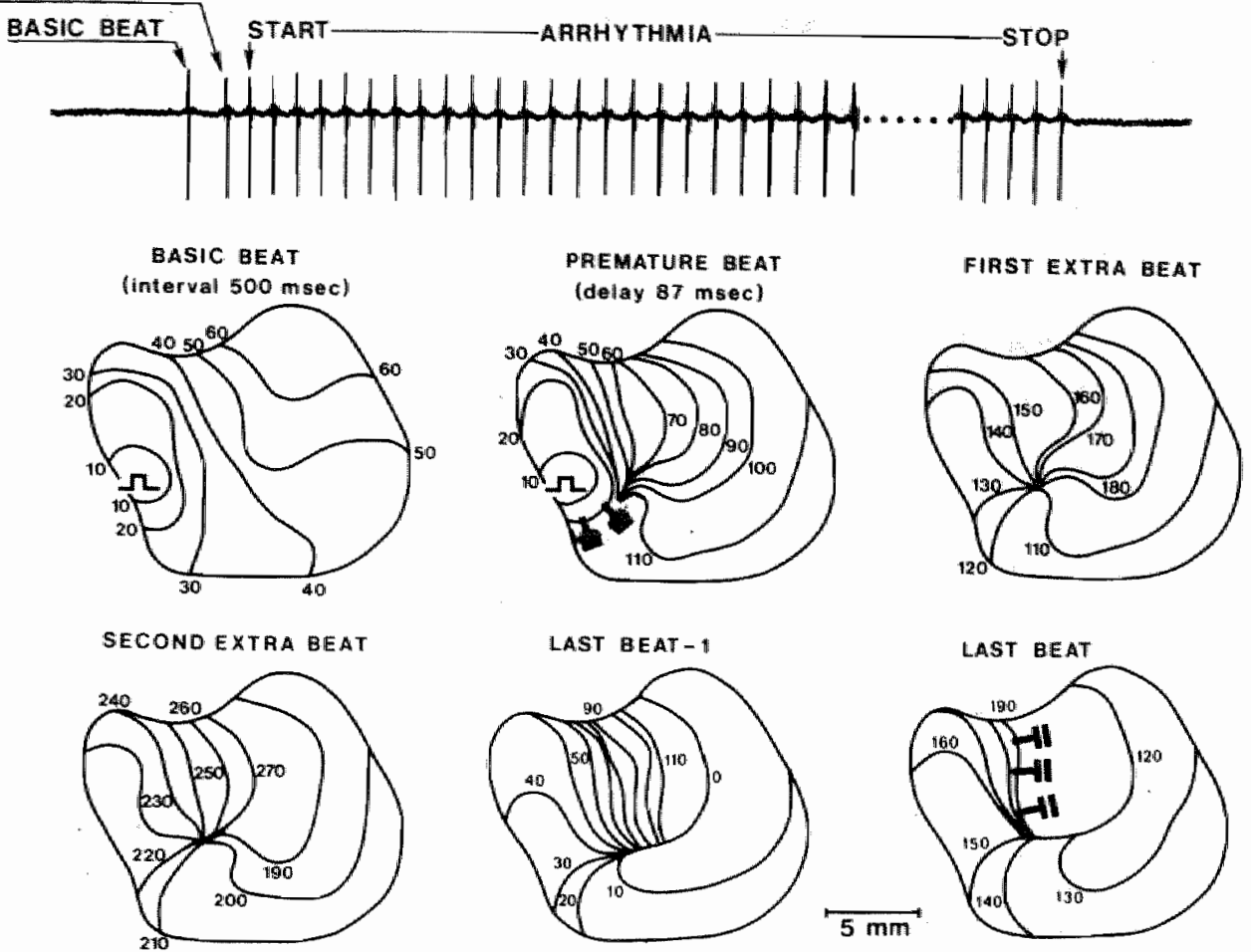

Figure 5.5. The activation sequence during the initiation and spontaneous termination of a tachy-arrhythmia in hypoxia. At the top of the figure, a local electrogram is depicted. The preparation was driven at a constant rate of $2 \mathrm{~Hz}(120$ beats/min $)$ and after 20 basic beats (of which the last beat is shown) a premature beat was induced with a coupling interval of $87 \mathrm{~ms}$ which was followed by a tachy-arrhythmia. In the lower part of the figure, the activation sequence of the last basic beat, the premature beat, the first two spontaneous beats and the last two spontaneous beats are drawn. Isochrones in the activation map of the basic activation are related (in milliseconds) to the moment at which the basic stimulus was given, while the isochrones in the maps of the premature activation, the first and the second spontaneous activations are related to the moment of the premature stimulus. In the last two panels activation times are related to an arbitrary moment $(t=0 ;$ several seconds after the initiation). The arrhythmia was terminated by block in the upper part of the preparation. See text for further description. 
activation maps.

During regular pacing there was a radial activation sequence of the preparation from the stimulus site travelling across the preparation and dying out at the upper right corner more than 60 ms after the initiation of the impulse. The conduction velocity was not homogeneous throughout the preparation. As can be seen from the close spacing of the isochrones, conduction in the left upper part of the preparation was less rapid than in the lower part.

During premature activation the conduction pattern was completely different. After the first $20 \mathrm{~ms}$ following the induction of the premature impulse a conduction block with a length of $4 \mathrm{~mm}$ occurred inferior to the stimulation site. In the upper part, conduction became much slower, as demonstrated by the clustering of the isochrones, but the wavefront was not blocked. After this slow propagation conduction velocity gradually increased and the wavefront rotated in a clockwise direction, reaching the area retrograde to the arc of conduction block $120 \mathrm{~ms}$ after the premature stimulus. As can be seen in the third map, the area proximal to the line of block by that time had restored its excitability and was reexcited by the turning wavefront. This first reentry occurred $130 \mathrm{~ms}$ after the initiation of the premature impulse. The wavefront then continued to circulate in the preparation in a clockwise direction, starting a second reentrant beat at $220 \mathrm{~ms}$ (fourth panel).

In the last two panels the activation sequence during the termination of the arrhythmia is drawn. Activation times start arbitrarily at $t=0 \mathrm{~ms}$ (about three seconds after the initiation). The impulse is still rotating in a clockwise direction, the cyclle length being increased to $120 \mathrm{~ms}$, while conduction in the upper left part of the preparation has become very slow. During the last beat the wavefront is abruptly blocked in this area of slow conduction thereby terminating the arrhythmia.

From these maps it is clear that the underlying mechanism of the arrhythmias in hypoxia is a circus movement of the impulse. Because no anatomical obstacle was present in the preparation this type of circus movement arrhythmia corresponds to the "leading circle" type of reentry (Allessie et al 1977).

From a total of 145 evoked arrhythmias, recorded in 7 experiments, the activation sequence of 25 tachy-arrhythmias was analyzed. In 14 cases intra-atrial reentry of the type shown in figure 5.5 could be demonstrated. In two cases the arrhythmia was caused by abnormal impulse generation, and in 9 cases the underlying mechanism could not be clearly defined. In these last cases 
TERMINATION OF REENTRY

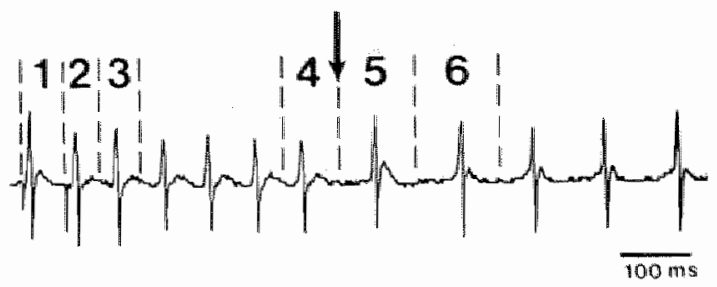

1 Basio geAT

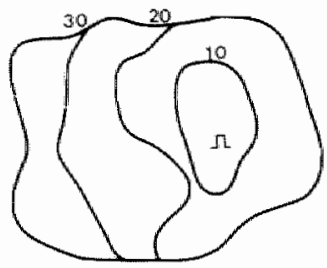

4 Last reentrant beat

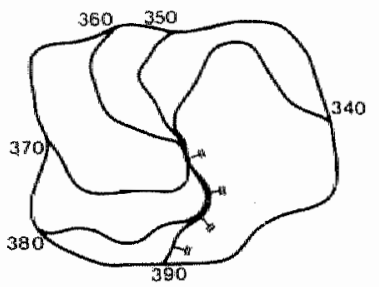

2 premature beat

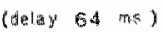

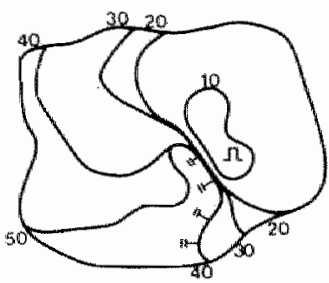

5 FiRSt fCCAL beAT

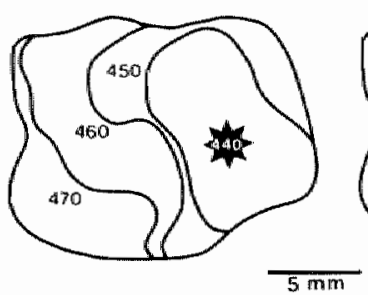

3 first reERTRANT Beat

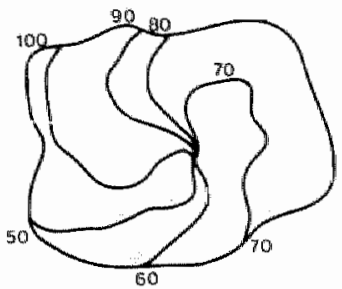

6 SECOND FOCAL BEAT

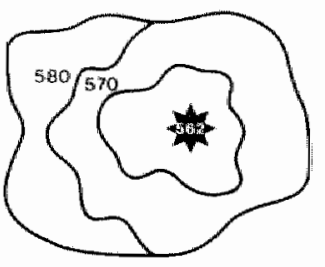

Figure 5.6. An example of two different mechanisms during the same arrhythmia. At the top of the figure a local electrogram depicts the basic beat (basic rhythm of $2 \mathrm{~Hz}$ ) and the premature beat (both preceded by a stimulus artefact) followed by ten spontaneous beats. There is a sudden change in interval in the middle of the tracing. The activation maps of the last basic beat, the premature beat and four spontaneous beats are drawn. Due to the occurrence of an area of conduction block during the premature activation (second panel) the impulse travelled in a counterclockwise direction. The activation pattern is depicted up to $t=50$ $m s$. In the third panel the activation spread is given between $t=50$ $\mathrm{ms}$ and $t=100 \mathrm{~ms}$ demonstrating reentry at $65 \mathrm{~ms}$. In the fourth panel the circulating impulse stopped spontaneously $390 \mathrm{~ms}$ after the arrhythmia had been started. This was however not the end of the arrhythmia. After $50 \mathrm{~ms}$ of quiescence a new activation appeared, activating the preparation radially (fifth panel). After this focal discharge another impulse emerged at the same site at $t=122$ ms (sixth panel). 
some part of the preparation had not been covered adequately by the recording electrode brush.

The arrhythmias which were caused by abnormal impulse generation were induced in preparations in which also intra-atrial reentry was demonstrated. Figure 5.6 depicts the spread of activation during an arrhythmia which is first based upon intra-atrial reentry and later caused by abnormal impulse generation. The local electrogram at the top of the figure shows a sudden change in interval. The first three activation maps depict the spread of activation during basic rhythm (first panel), the premature beat (second panel) and the first intra-atrial reentry (third panel). The activation maps of the second, third and fourth beat are skipped because they were repetitions of the first circus movement beat.

During the fifth beat " of the tachycardia (fourth panel), the spread of excitation was blocked halfway the preparation $390 \mathrm{~ms}$ after the induction of the arrhythmia and the circus movement was terminated. However, after a $50 \mathrm{~ms}$ period of quiescence a new impulse originated from the area of the original stimulus site. No electrical activity could be demonstrated between the moment of block of the circus movement arrhythmia and the start of this new impulse. The subsequent spread of excitation differed completely from that during reentry. During abnormal impulse formation the wavefront spread in a radial fashion, although during the first beat there was an area of relatively slow conduction where the circulating impulse had stopped because this area was still in its relative refractory period. New spontaneous impulses continued to originate from the same site and the spread of excitation became more and more homogeneous with conduction velocity increasing.

In conclusion the majority of tachy-arrhythmias induced in hypoxia with a single premature impulse were caused by intra-atrial reentry in the absence of an anatomical obstacle. In a minority of cases abnormal impulse formation was found.

\subsection{Why is it easier to initiate intra-atrial reentry in hypoxia?}

To study the factors responsible for the higher vulnerability to arrhythmias in hypoxia, we measured several electrophysiological properties during control and during hypoxia. The refractory period was measured in 20 isolated left atria of the rabbit. A stimulus electrode was positioned on the endocardial surface of the prepa"ration while a recording electrode was located within a few millimeters distance. Cathodal stimuli of $1 \mathrm{~ms}$ duration were used 
both during basic rhythm (2 $\mathrm{Hz})$ and during premature stimulation. Diastolic threshold was measured every 5 minutes during control and during hypoxia by gradually increasing the stimulus strength while pacing at $2 \mathrm{~Hz}$ until capture occurred. Refractory period was measured using premature stimuli with a strength of four times diastolic threshold induced after 20 basic beats. The coupling interval was gradually increased in steps of $2 \mathrm{~ms}$ from a very short value, falling well within the absolute refractory period, until a premature activation was observed at the recording electrode. The shortest possible coupling interval which induced premature activation was taken as the effective refractory period. This procedure was repeated every 5 minutes during control and during hypoxia.

Figure 5.7 panel A presents the influence of hypoxia on the diastolic threshold. During control diastolic threshold had a mean value of 60 microamp which increased during hypoxia $(81,72,68$ microamps at 15,30 and 45 minutes respectively). At two sites the threshold for stimulation increased so much that stimulation became impossible (arrows). The refractory periods are plotted in panel B and demonstrated a biphasic pattern with an increase from a mean of $67 \mathrm{~ms}$ ( $\pm 15 \mathrm{~ms}$ ) during control to a mean of $87 \mathrm{~ms}( \pm 25 \mathrm{~ms})$ at 10 minutes in hypoxia. This was followed by a decrease in the refractory period reaching a mean of $66 \mathrm{~ms}( \pm 17 \mathrm{~ms})$ at 30 minutes hypoxia. In panel $\mathrm{C}$, the relative changes in refractory period compared to control are plotted. The refractory period was only significantly increased during the first 15 minutes of hypoxia $(p<0.001)$. After that period no significant differences from control were present.

Conduction velocity was measured in 15 experiments using the atrial strip preparation (see figure 3.7, chapter III). The preparations were stimulated at a rate of $2 \mathrm{~Hz}$ through a stimulus electrode located at one end of the strip and conduction was measured from the interval in activation at the two recording electrodes. The proximal electrode was located in the vicinity of the stimulus electrode while the distal electrode was located at the other end of the preparation. The results are displayed in panel $\mathrm{D}$ of figure 5.7. Hypoxia induced a significant decrease in conduction velocity $(\mathrm{p}<0.001)$ ranging between $15 \%$ and $35 \%$ during the 60 minutes hypoxic period.

In four experiments the effect of hypoxia on premature conduction was measured. The results of one experiment are displayed in figure 5.8. In the inset, the effect of hypoxia on the conduction time during basic rhythm $(2 \mathrm{~Hz}$ ) is shown. During control (curve 1) 
and subsequently during bypoxia (curves 2, 3 and 4) premature impulses with coupling intervals ranging from $300 \mathrm{~ms}$ down to the smallest possible interval were evoked. The measured conduction times are plotted on the ordinate with the corresponding coupling intervals on the abscissa. During control, conduction times remained constant at coupling intervals between 150 and $300 \mathrm{~ms}$ but gradually increased (conduction velocity decreased) at coupling intervals shorter than $150 \mathrm{~ms}$. After 19 minutes in hypoxia (curve 2) the curve has shifted upwards to longer conduction times (=sllower conduction velocities) and also to the right due to an increase in refractory period. After 33 minutes, conduction velocity was slightly restored as shown by the slight downwards shift of the curve (no. 3) to lower conduction times but, after 49 minutes in hypoxia, conduction velocity was further depressed (curve 4).

From the results of these experiments, the influence of hypoxia on the wavelength can be estimated. For example from the data presented in figure 5.8 the refractory period increased from 81 to 100 at 19 minutes hypoxia $(24 \%)$ while the basic conduction time increased from $57 \mathrm{~ms}$ to $68 \mathrm{~ms}$ (a decrease in conduction velocity of $19 \%$ ). Because the wavelength is the product of the refractory period and the conduction velocity, the wavelength of the basic beat is only slightly lengthened at 19 minutes. At $33 \mathrm{~min}$ in hypoxia, refractory period has increased with $11 \%$ while conduction velocity has decreased by $16 \%$. At 49 minutes the increase in the refractory period $(11 \%)$ was considerably smaller than the decrease in conduction velocity $(32 \%)$ implicating that, after prolonged hypoxia, the wavelength will be shortened.

Although we did not measure the wavelength of the premature impulse, sufficient evidence is available to warrant the conclusion that also during premature activation a depression of the conduction velocity is counteracted by an increase in the refractory period, at least for a substantial part of the hypoxic period. The

Figure 5.7. The effects of hypoxia on the threshold for electrical stimulation, refractory period and conduction velocity. In panel $A$ the amount of current necessary to initiate a propagated response in rabbit left atrial tissue was repeatedly measured at 20 different sites in nine experiments both during control and during the first 60 minutes in hypoxia. Under control conditions the threshold waried between 18 and 105 micro-amperes, whereas during hypoxia it ranged from 30 micro-amperes up to several milli-amperes. In two cases the atrium became locally inexcitable (arrows). In panels 


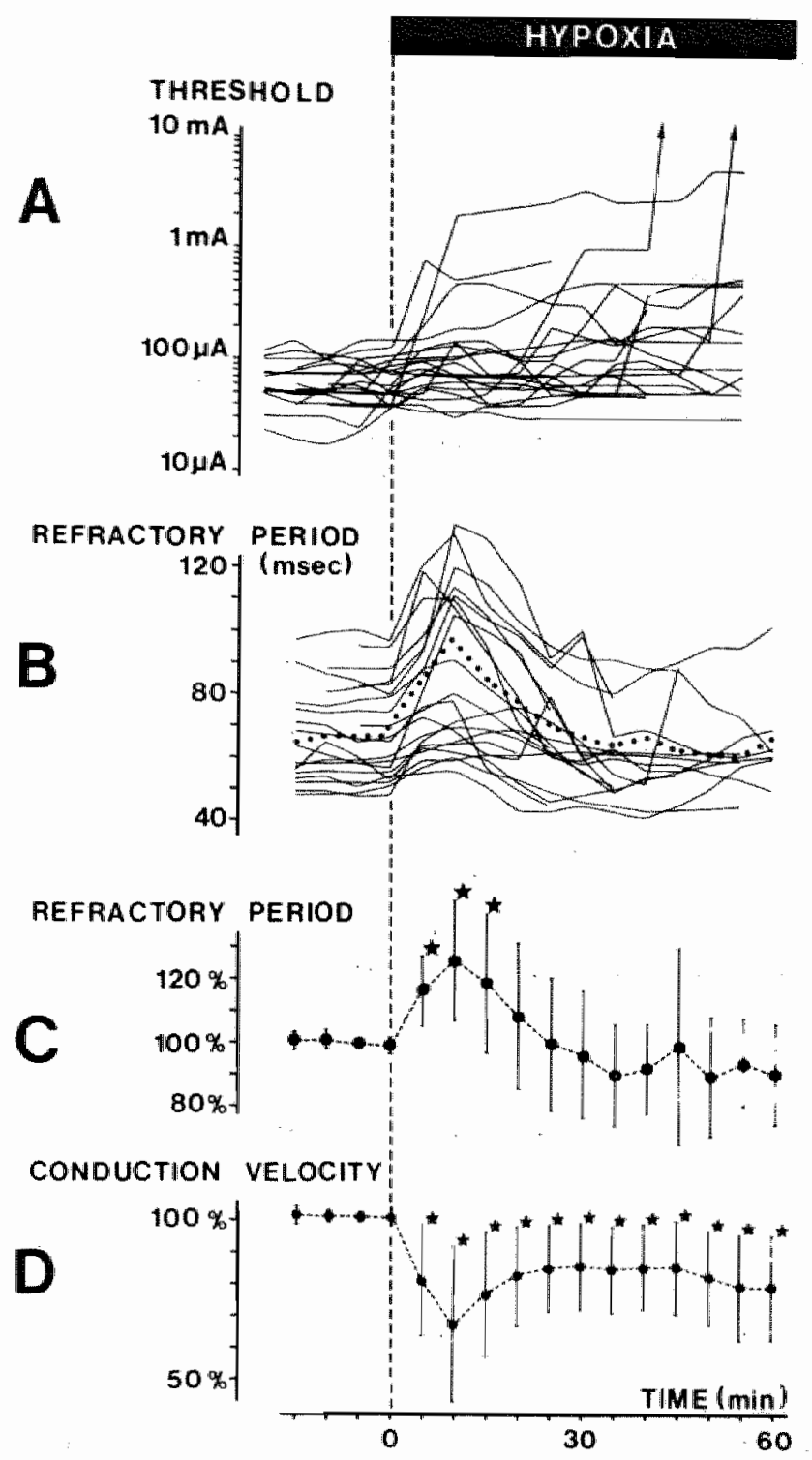

$B$ and $C$ the changes in refractory period are plotted in milliseconds and as a percentage of control. The stippled curve in panel $B$ represents the mean values. Both plots demonstrate a transient increase in refractoriness during the first 15 minutes in hypoxia. In panel $D$ changes in conduction velocity, as measured in atrial strip preparations between two extracellular electrodes, are plotted. During hypoxia, conduction velocity was significantly depressed throughout the 60 minutes period of hypoxia $\left(^{*}=p<0.001\right)$. 


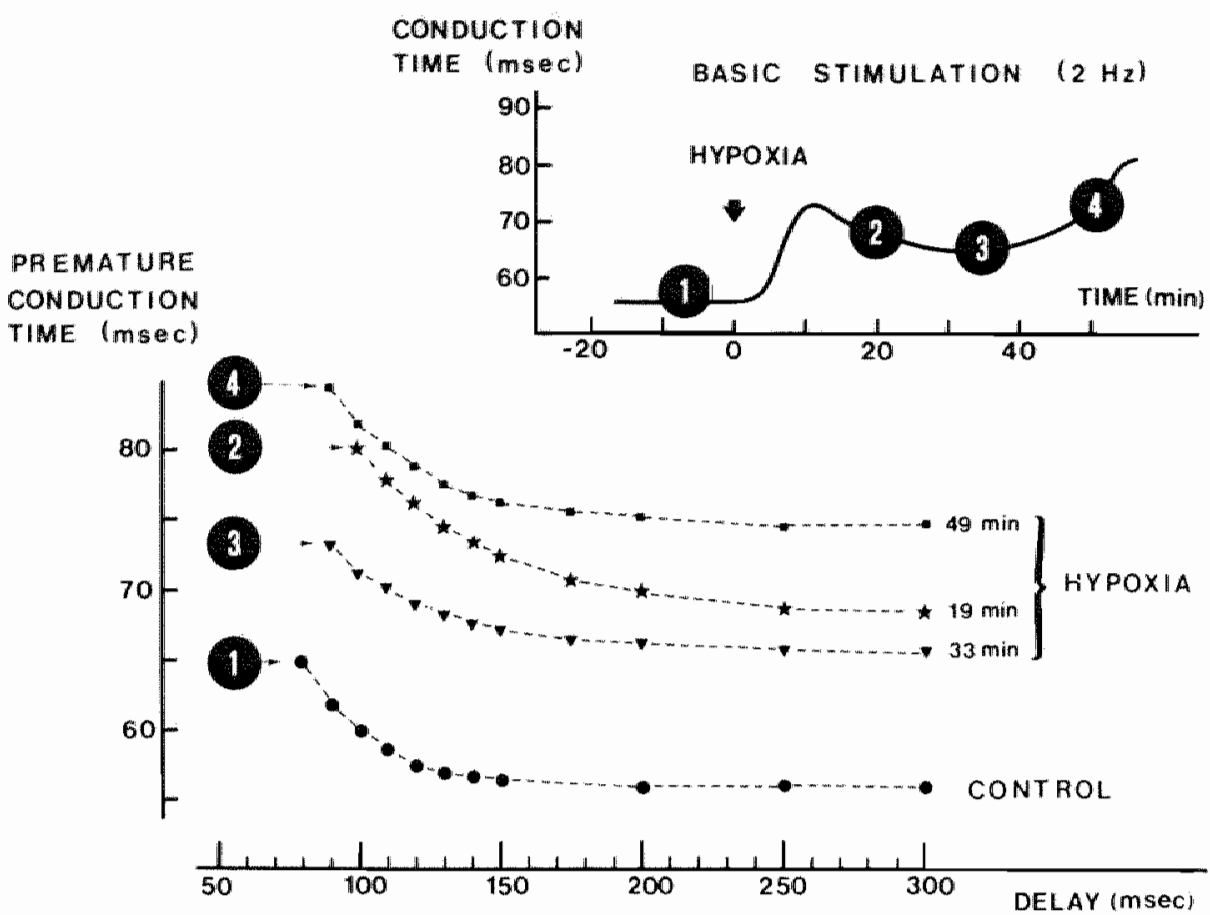

Figure 5.8. The effect of hypoxia on premature conduction. The time necessary for $a$ premature impulse to travel between two surface electrodes in an atrial strip, was measured at different prematurities. On the horizontal axis, the coupling interval between the last basic beat (basic rhythm of $2 \mathrm{~Hz}$ ) and the premature impulse is given in ms. The relation between the coupling interval and premature conduction was determined during contral (serie 1) and during hypoxia (series 2, 3 and 4). During control premature conduction is stable at long coupling intervals. At coupling intervals shorter than $120 \mathrm{~ms}$ conduction time increases ( $=$ conduction velocity being the reciprocal decreases) until a maximum walue is reached at the shortest possible prematurity. In hypoxia the relation shifts to longer conduction times $(=$ slower conduction velocities). Premature conduction velocity is not stable during hypoxia but increases at 33 minutes and furter decreases at 49 minutes hypoxia.

arrhythmogenic effect of hypoxia however was present during the whole hypoxic period (figure 5.1). We must therefore conclude that "changes in the wavelength cannot be held responsible for the higher vulnerability to arrhythmias during hypoxia. 


\section{The effects of hypoxia on the transmembrane potential.}

To get more detailed data on the influence of hypoxia microelectrode impalements were performed in the same experiments in which conduction velocity was measured. The parameters which underly the changes in conduction velocity, such as the membrane resting potential, the action potential amplitude and the upstroke velocity were measured (figure 5.9). After 10 minutes in hypoxia there was a decrease in resting potential (from $-77 \pm 3 \mathrm{mV}$ to $-71 \pm 3$ $\mathrm{mV}, \mathrm{p}<0.001$ ), in amplitude of the action potential (from $100 \pm 8 \mathrm{mV}$ to $85 \pm 11 \mathrm{mV}, \mathrm{p}<0.001$ ) and in upstroke velocity (from $218 \pm 92 \mathrm{~V} / \mathrm{sec}$ to $154 \pm 74 \mathrm{~V} / \mathrm{sec}$ ). At that moment the conduction velocity was depressed by $32 \%$. After this initial effect all action potential parameters returned to control values together with an increase in conduction velocity. Interestingly, conduction velocity remained significantly depressed aithough the effect was small. This could be due to an increase in the resistance of intercellular junctions as shown by Wojtcak (1979). In conclusion, the changes in conduction velocity during hypoxia were paralleled by concomittant changes in the underlying cellular electrophysiology.

In the preceding paragraph, a transient increase in the refractory period by hypoxia was described. One of the underlying factors influencing refractoriness is the action potential duration. As shown in figure 5.10, the action potential duration in our preparation decreased from the onset of hypoxia while at the same time refractoriness was transiently increased. This implies that in hypoxia the refractory period outlasts full repolarization. We can therefore confirm the suggestion of linuma et al. (1978) that there is an increase in "post-repolarization refractoriness" during the first 20-30 minutes in hypoxia.

\subsection{Discussion.}

Reentrant arrhythmias could be initiated much more easily in hypoxia than under normal experimental conditions (figure 5.1). We investigated whether a shortening of the wavelength, which was responsible for the higher inducibility of tachy-arrhythmias in low-potassium (chapter IV), also occurred in hypoxia. A shortening of the wavelength could be caused by a shortening of the refractory period, a depression of the conduction velocity or both. 


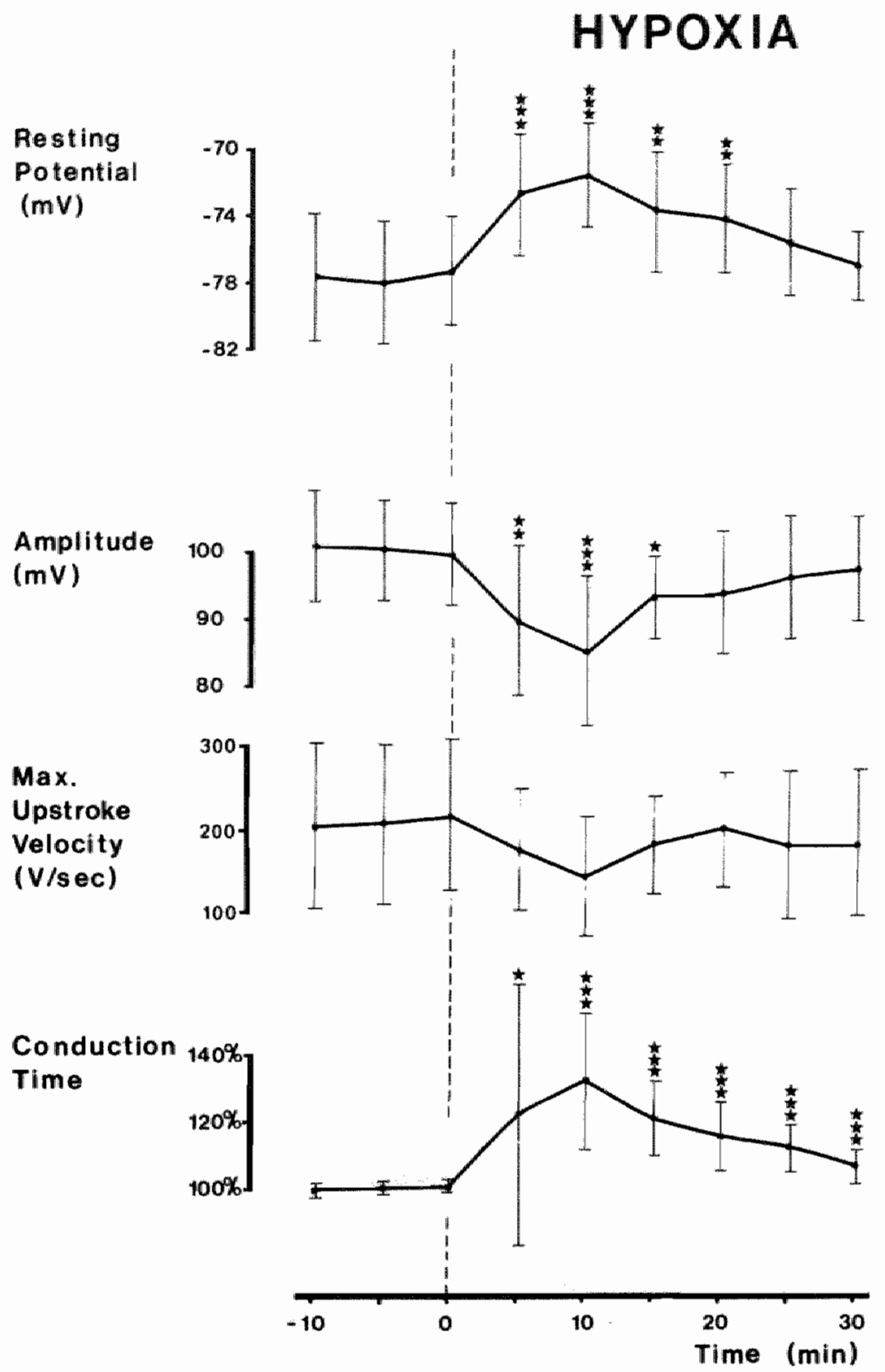

Figure 5.9. The effects of hypoxia on the resting potential, the amplitude and the maximum upstroke velocity of the action potential and on conduction velocity in rabbit atrial myocardium. In 22 experiments a stable impalement was achieved during control and subsequent hypoxia. All values were measured at 5 minutes interval and the means and standard deviations of the values are plotted. Hypoxia decreased the resting potential, the amplitude of the action 
Refractoriness and Hypoxia.

At first glance the reports in the literature concerning the effects of hypoxia on the refractory period seem to disagree with our own results. In 1925 Resnik measured the refractory period of atrial tissue in anesthetized dogs that were atropinized and had their vagal nerves tied off. $\mathrm{He}$ found a decrease in refractoriness when the oxygen saturation was lowered. Harris and Matlock (1947) measured refractoriness in the ventricles of the anesthetized dog with intact innervation. In moderate hypoxia, there was no influence on the refractory period but in severe hypoxia the refractory period decreased by 35 milliseconds. Vleugels and Carmeliet (1976) have measured a decrease of the refractory period in the embryonic chick heart in hypoxia. Prasad (1975) measured the refractory period in human papillary muscle and found a decrease after 60 minutes in hypoxia. Ravens and Ravens (1976) reported a decrease of the refractory period in isolated papillary muscle of the guinea pig. linuma et al. (1978), performing experiments on canine papillary muscle, found the effective refractory period (as measured by using test stimulus strength of five times threshold) to be shortened in hypoxia, whereas the total refractory period (using a stimulus strength of threshold value) was lengthened. Lazzara et al. (1978) measured strength-interval curves in canine endocardial preparations and found an increase in refractoriness in hypoxia.

Our results demonstrate a transient increase in the refractory period during the first 20-30 minutes of hypoxia, followed by a decrease to values slightly less than control values. It is essential that the refractory period was repeatedly measured in our experiments, every 5 minutes, during the first 60 minutes of hypoxia. In the reviewed literature the duration of the hypoxic period, at which the measurements of refractoriness were made, is not mentioned except for Prasad (1975) who found a decrease at 60 minutes hypoxia. According to our data the moment of the measurement is important. If the refractory period is measured soon after switching to hypoxia an increase in refractoriness is found while later a de-

potential, the maximum upstroke velocity together with a decrease in the conduction velocity. This effect lasted only a short time; all action potential parameters returned to control within 30 minutes of hypoxia $\left({ }^{*}=p<0.05 ; * *=p<0.01 ; * * *=p<0.001\right)$. 


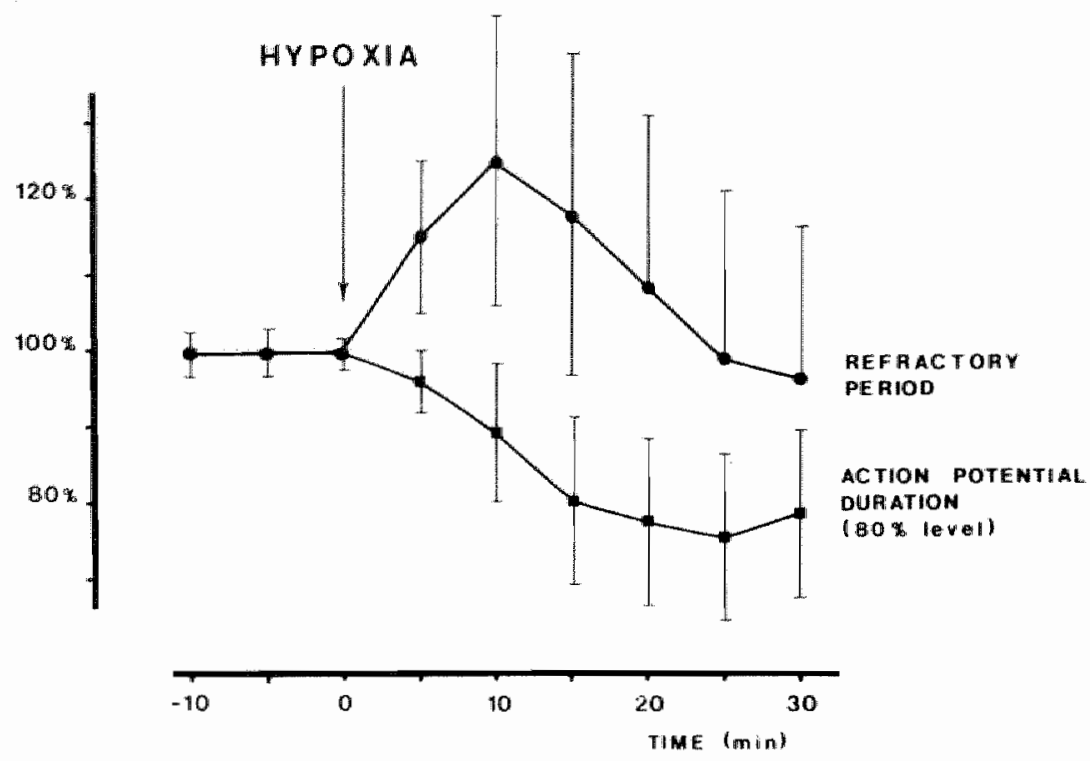

Figure 5.10. The relation of action potential duration in rabbit atrial fibers and refractoriness in hypoxia. Action potential duration was measured at $80 \%$ repolarization at 5 minutes interval in control and subequently during 30 minutes hypoxia $(n=22)$. The values of the refractory period are the same as those displayed in figure 5.7. At the start of hypoxia there is an immediate and significant $(p<0.001)$ shortening of the action potential duration while refractoriness is transiently increased, thereby inducing a clear postrepolarization refractoriness.

crease in refractoriness can be measured. It is also important to appreciate the individual variations of different sites and preparations to hypoxia, as examplified by the increase in the standard deviation in figure 5.7 panel $C$. This variability made it necessary to perform a large number of experiments. Our conclusion that the transient, but significant, increase in the refractory period in hypoxia is followed by a decrease to a slightly lower value than control holds as a general rule. In individual cases a constant increase or an immediate decrease of the refractory period has also been observed. 
Conduction Velocity and Hypoxia.

There are several reports in the literature on the effects of hypoxia on conduction velocity. Harris and Matlock (1947) studied the influence of hypoxia on conduction in anesthetized dogs in situ with an intact innervation. Conduction velocity was estimated from the latency between the stimulus and the recorded electrical activity on a local electrogram and from the PR interval and the duration of the QRS complex in lead II of the electrocardiogram. Moderate hypoxia caused an increase of conduction velocity but this might be mediated by an increase of the sympathetic tone. Severe hypoxia caused a slowing of conduction velocity. Trautwein et al. (1954) measured the effects of hypoxia on the conduction velocity in isolated canine Purkinje fibers and feline papillary muscle; in these preparations they found a reduction of the conduction velocity only if hypoxia was prolonged for more than one hour or if several hypoxic episodes were repeated. Webb and Hollander (1956) on the other hand did show a decrease in conduction velocity in rat atria of $36 \%$ after 17 minutes hypoxia. Hondeghem et al. (1974) demonstrated in guinea-pig papillary muscle a decrease of the upstroke velocity, a parameter often used as an indicator of conduction velocity, from 280 to $220 \mathrm{~V} / \mathrm{sec}$ in hypoxia. This effect of hypoxia was also described by Prasad (1975) in human papillary muscle. Nishi et al. (1977) described in an isolated right atrium preparation of the rabbit that reduction of the oxygen content below $10 \mathrm{mmHg}$ induced conduction blocks in the atrial tissue within 15 minutes. But recently Senges et al. (1979) reported that the conduction velocity as measured in the crista terminalis of the rabbit did not change significantly in hypoxia.

Our results demonstrate that conduction velocity is significantly depressed during the first hour in hypoxia. The amount of depression however is slight, varying between $10 \%$ and $40 \%$ of control. There is also, as is the case for the refractory period, an increase in variability in conduction velocity in hypoxia. This might explain that this slight and variable depression in conduction may not always reach statistically significant values.

The arrhythmogenic nature of hypoxia seems to be essentially different from the one described in chapter IV; at low potassium the higher vulnerability to reentrant arrhythmia was caused by a clear reduction in refractoriness, together with a reduction in conduction velocity. These two factors will lead to a marked shortening of the wavelength which is shown to facilitate reentry 
(Rensma 1987). During hypoxia the wavelength was not consistently reduced and was sometimes even lengthened. Nevertheless it was always possible to initiate tachy-arrhythmias. Therefore during hypoxia a shortened wavellength is not the primary cause for the higher incidence of reentrant arrhythmias. 


\section{Chapter VI \\ SPATIAL DISPERSION IN REFRACTORINESS AND CONDUCTION IN HYPOXIA.}

\subsection{Introduction.}

In the previous chapter, the arrhythmogenic property of hypoxia could not be explained by a possible shortening of the wavelength. Durizg hypoxic superfusion however, both the refractory period measurements and the determinations of the conduction velocities showed a higher variability than during control (figure 5.7 panel $C$ and D). This indicates that hypoxia may cause increased inhomo-
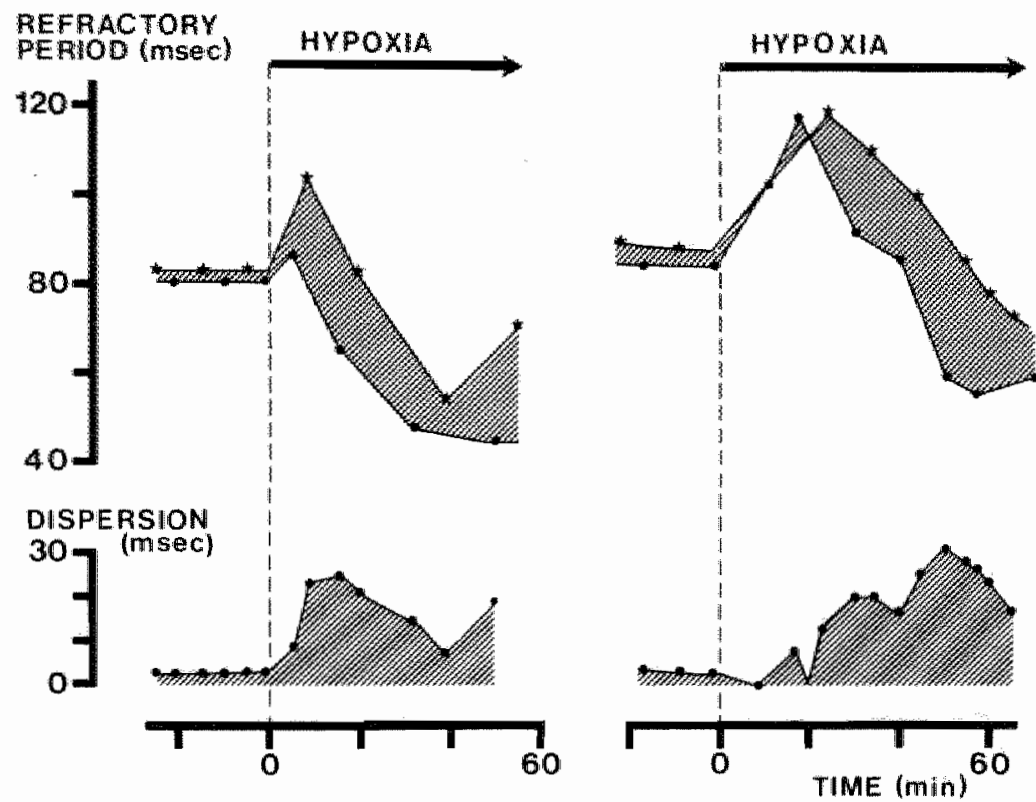

Figure 6.1. Two examples of increased dispersion in refractoriness in hypoxia. The refractory period was measured at two sites (located at 10 and $5 \mathrm{~mm}$ distance respectively) during control and during 60 minutes hypoxia. In the lower panels the difference of the refractory period as measured at the two sites are plotted. In both cases the dispersion during control was small (resp. 3 and 5 ms) but increased markedly during hypoxia, up to $30 \mathrm{~ms}$. 
geneity in the conduction of a premature impulse. In this chapter experiments are described which were undertaken to determine whether hypoxia induced an increased spatial dispersion in refractory period or an increased inhomogeneity in conduction. Such increased heterogeneity might lead to a higher incidence of conduction blocks and a higher chance of reentrant arrhythmias.

\subsection{Spatial dispersion of the refractory period.}

Refractory periods were measured at several sites during the same hypoxic episode in 4 experiments. The results of two experiments are displayed in figure 6.1. In the upper panels the values of the refractory period as measured at two different sites during control and during 60 minutes hypoxia are plotted. The electrodes were positioned at a distance of 10 and $5 \mathrm{~mm}$ respectively. During control the refractory period ranged between 81 and $84 \mathrm{~ms}$ in the left panel and between 85 and $90 \mathrm{~ms}$ in the right panel. In the lower panels the dispersion in the refractory period is plotted as the difference between the two sites. The dispersion increased from a control value of $3 \mathrm{~ms}$ in the left panel and $5 \mathrm{~ms}$ in the right panel to much higher values during hypoxia. It must be stressed that these measurements only represent a small sample of the total spatial dispersion. Because measuring refractory periods takes time and because the situation is not stable during hypoxia, we can only measure the refractory period at a few sites. However, even from these few samples, it is obvious that the spatial dispersion in refractoriness increased markedly in hypoxia.

Figure 6.2. The importance of dispersion in refractoriness for the induction of a reentrant beat. In the upper part of the figure the refractory periods, as measured at two different locations before and during hypoxia, are plotted. At one location the refractory period gradually declined during hypoxia (asterisks) while the ocher site demonstrated a transient increase (squares). At the moment of maximal dispersion (20 minutes in hypoxia) a premature beat elicited with $a$ coupling interval of $64 \mathrm{~ms}$ was followed by a reentrant beat. As shown by the electrograms in the middle panel, a premature stimulus induced a few minutes before or after this moment did not elicit a spontaneous beat. In the lower part of the figure the activation maps of the basic beat, the premature beat and the spontaneous beat are drawn. The first map demonstrates 


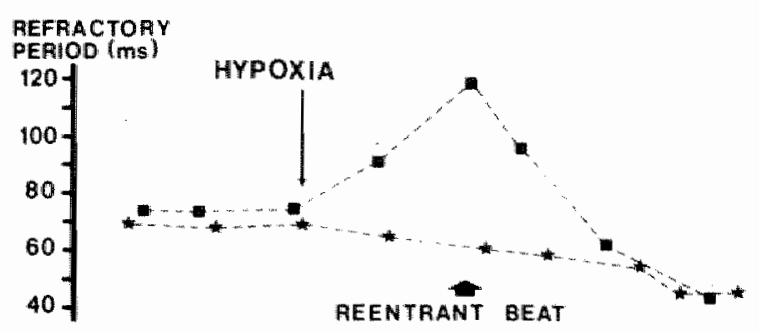

$18 \mathrm{~min}$
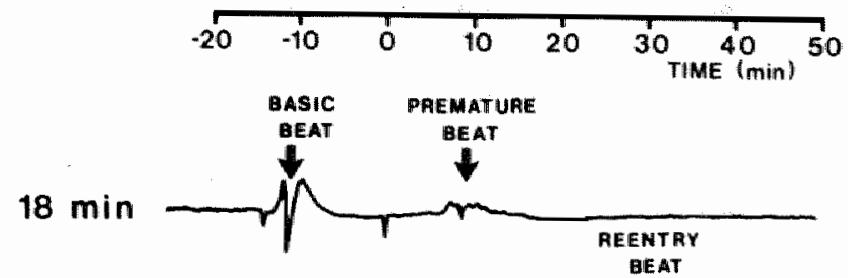

$20 \min$

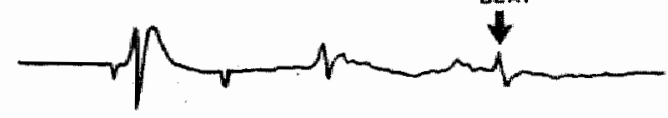

$23 \mathrm{~min}$

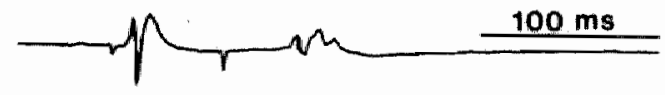

BASIC BEAT

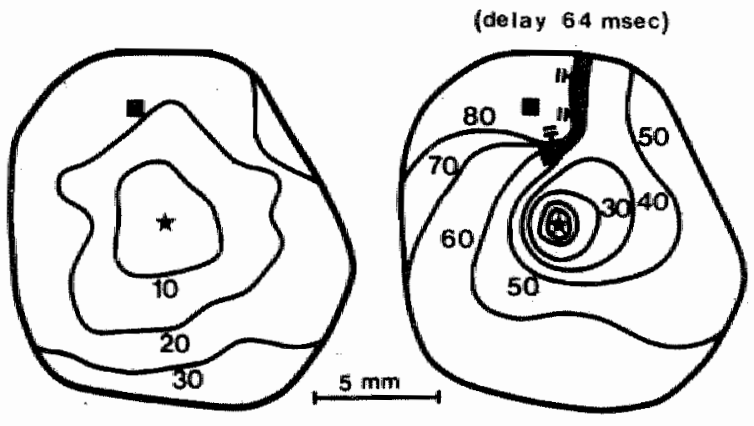

REENTRANT BEAT

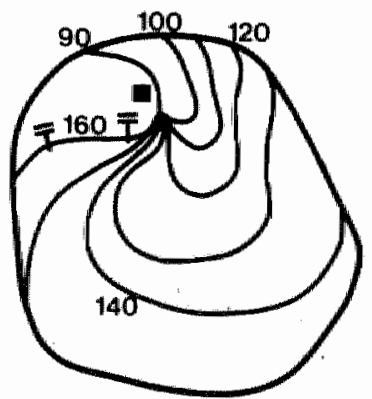

the radial spread of excitation following a basic stimulus (rhythm of $2 \mathrm{~Hz}$. In the second map the activation of a premature impulse is depicted. The premature spread of excitation is blocked in the vicinity of the second measuring electrode (square) at $50 \mathrm{~ms}$. The impulse circumvents this block and rexcites the area which was previously excited by the premature impulse at $100 \mathrm{~ms}$, thereby inducing the reentrant beat (third map). 
The importance of the increase in dispersion in local refractoriness is illustrated in one experiment in which measurement of the refractory period was combined with the mapping of the activation sequence (figure 6.2). The refractory period as measured at two different sites is plotted in the upper panel. While the refractory period increased transiently during hypoxia at one site (squares), the second site showed a slow decrease (asterisks). At the moment of maximal dispersion between the two sites $(60 \mathrm{~ms})$, after 20 minutes of hypoxia, a premature stimulus delivered at the site with the shortest refractory period induced a reentrant beat. As shown by the recordings in the middle panel it was not possible to initiate the arrhythmia a few minutes before or after this moment. The activation maps during the initiation of the reentrant beat are displayed in the lower panels. The sites where the refractory periods were measured are depicted by the square (long refractory period) and the asterisk (short refractory period) in the maps. During the activations displayed in the maps, the preparation was driven from the site marked by the asterisk. During a basic beat (first activation map) the spread of excitation was essentially radial. The second map depicts the activation sequence induced by a premature stimulus initiated $64 \mathrm{~ms}$ after the previous basic beat. After $50 \mathrm{~ms}$ the wavefront blocks at an area which is still refractory. The arc of conduction block extended to the upper border of the preparation. At the left side of the arc the wavefront could circumvent this area of block and reached the retrograde area 100 $\mathrm{ms}$ after the initiation of the premature impulse. A reentrant beat ensued as demonstrated by the activation map of the spontaneous beat.

\subsection{Quantification of conduction blocks.}

If an increase in spatial dispersion plays a major role in the

Figure 6.3. Quantification of conduction blocks in activation maps. In the two upper panels premature activations elicited at coupling intervals of 100 and $85 \mathrm{~ms}$ are depicted. Isochrones are drawn around areas activated within time steps of 10 milliseconds. As can be visualized from the spacing of the isochrones, conduction is slowed and even blocked at some sites. The same activation times are repeated in the middle panels but without the isochrones. Instead, straight lines, representing local conduction blocks are 


\section{QUANTIFICATION OF CONDUCTION \\ BLOCKS}

delay $100 \mathrm{~ms}$

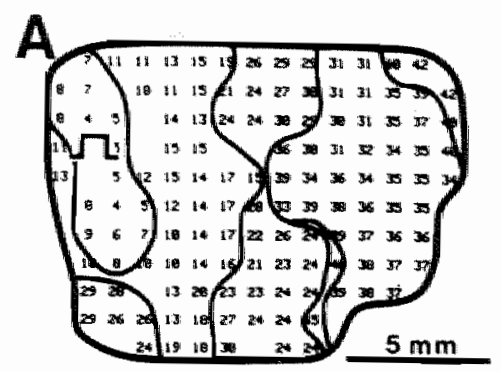

B

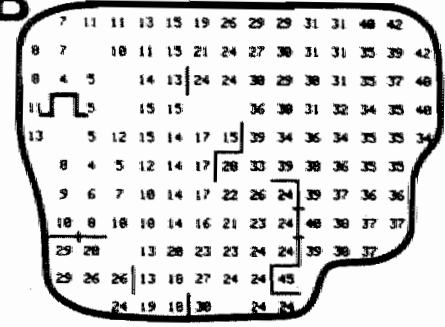

NUMBER

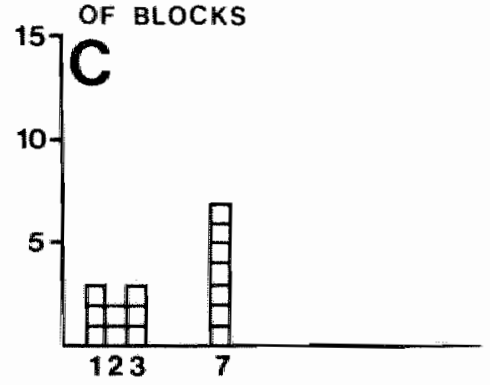

TOTAL 15 delay $85 \mathrm{~ms}$
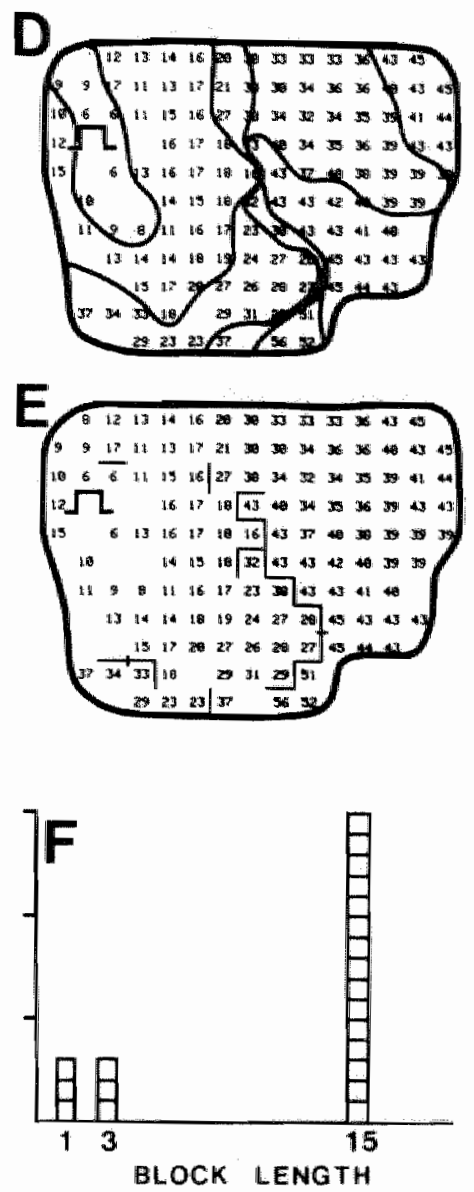

21

drawn $(=$ block-unit). Conduction block between two recording sites is assumed when the difference in activation times between the two recording electrodes is langer than 10 milliseconds (conduction velocity slower than $10 \mathrm{~cm} / \mathrm{sec}$. In the lower panels, the number of block-units are plotted. The length of the arcs of conduction blocks and the number of conduction blocks increased at shorter coupling intervals. 
induction of intra-atrial reentry there should be an increase in the inhomogeneity of conduction of the premature impulse. As a first step to measure the degree of conduction inhomogeneity during premature activation, we quantified the occurrence of local conduction blocks during propagation of early premature beats.

An example of the method is demonstrated in figure 6.3. Panel A depicts the spread of excitation of a premature beat during hypoxia, initiated at the site indicated by the stimulus symbol, with a coupling interval of $100 \mathrm{~ms}$. The plotted values correspond to the local activation times in relation to the moment of premature stimulation. Isochrones are drawn in steps of $10 \mathrm{~ms}$ to visualize the spread of premature excitation. In the middle lower part of the preparation, two isochrones are clustered together suggesting an area of conduction block.

In panel B the activation map is repeated without the isochrones. The locations of local conduction blocks are displayed by short straight lines. The presence of a local conduction block was determined in the following way. The difference in activation time between each pair of adjacent electrodes was calculated. When propagation is rapid, small differences in activation time will exist. If conduction block occurs between two adjacent electrodes, a larger difference in activation time will be measured.

A large difference in activation time between two adjacent electrodes can also be caused by slow conduction. A minimal value of $10 \mathrm{~cm} / \mathrm{sec}$ was chosen as the criterion for conduction block. Because the inter-electrode distance in figure 6.3 was 1 millimeter, a difference in activation time between adjacent electrodes of 10 or more milliseconds indicates conduction block. These sites are marked by a straight line between the two adjacent recording sites ( $=$ block-unit). In panel $B$ several smaller areas of conduction blocks were identified. In the lower middle part of the preparation a longer arc of conduction block was present. In the lower panels of the figure histograms are displayed which give the number of conduction blocks at different length. In this way, during the premature beat with a coupling interval of $100 \mathrm{~ms}$, a total of 15 block-units is found.

In the right part of the figure the same procedure was repeated for a premature activation with a shorter coupling interval $(85 \mathrm{~ms})$. As depicted in panel $D$ there was an increase in the isochrone clustering in the middle lower part of the preparation suggesting an increase in the degree of conduction block. In the middle right panel the calculation of conduction blocks was repeated using the same criterion of $10 \mathrm{~cm} / \mathrm{sec}$. The longer arc of conduction block 
was extended and now consisted of 15 block-units. The corresponding histogram (panel F) also demonstrates this increase in local conduction block (total of 21 block-units).

Using this procedure, the number of conduction blocks during premature activation was measured at several moments during control and during hypoxia. The results of three experiments are displayed in figure 6.4. The preparation was stimulated from one site and the refractory period at that site was continuously measured. The drawn line represents the time course of the refractory period which in this case (panel A) shortened gradually from $85 \mathrm{~ms}$ to $45 \mathrm{~ms}$. At several moments during control and during hypoxia, activation maps were made of premature activations with coupling intervals ranging from the refractory period to a coupling interval of $150 \mathrm{~ms}$. From these maps, the total number of conduction blocks was calculated.

In the experiment presented in panell $A$, the largest number of conduction blocks (10) during control was found when a premature activation was initiated at the shortest possible coupling interval $(85 \mathrm{~ms})$. Increase of the coupling interval resulted in a decrease of the number of conduction blocks as can be expected because the preparation had more time to recover from the previous basic activation. During hypoxia the number of conduction blocks increased considerably. At the same coupling interval of $85 \mathrm{~ms}$ the number of blocks increased from 10 during control to 27 at 15 minutes, to 12 at 30 minutes and to 21 at 50 minutes of hypoxia. Because we measured conduction blocks at a large number of coupling intervals throughout the hypoxic period we could delineate a zone (hatched area) in which premature activations induced more conduction blocks than the maximal number during control (10).

The incidence of conduction blocks was also measured in two additional experiments and the results are displayed in panell B and $C$ (figure 6.12). In each case the hatched area encompassed those premature activations which induced a higher number of blocks than the maximal number during control. In each experiment hypoxia induced a marked increase in the occurrence of conduction blocks during premature activation. We believe that this increase in inhomogeneity in conduction is responsible for the arrhythmogenic property of hypoxia.

\subsection{Discussion.}

In the previous chapter we have shown that changes in wave- 
length during hypoxia are probably not responsible for the arrhythmogenic nature of hypoxia. In this chapter, hypoxia was found to increase spatial dispersion in recovery, together with a higher incidence of local conduction blocks. The spatial distribution of electrophysiological factors such as refractoriness and conduction velocity in cardiac tissue has been studied by several investigators. Alessi et al. (1958) measured the refractory period at several locations in the right atrium of the anesthetized dog and found a range of 40 milliseconds during control situation. Stimulation of the vagal nerves caused a shortening of the average refractory period whereas the range markedly increased. Concomitant with these changes, there was an increase in the likelihood of initiating atrial fibrillation. Han et al. (1964) measured refractoriness in ventricular tissue of the anesthetized $\operatorname{dog}$ and found a range in refractoriness during basic thythm of 12 milliseconds. This dispersion increased to 28 milliseconds following an early premature beat while the mean refractory period was reduced. Ischaemia further reduced the mean value and further increased the dispersion in refractoriness. In the present study we found that the dispersion in spatial distribution in refractoriness is increased by hypoxia.

Spatial inhomogeneity in conduction has also been studied. Draper and Mya-Tu (1959) demonstrated in papillary muscle and in the sulcus terminalis of the cat and goat heart that conduction velocity is higher when the impulse is travelling parallel to the fiber orientation and that conduction becomes slower when travelling in the transverse direction. This inhomogeneity in conduction is based on the underlying cellular architecture of the myocardium and has

Figure 6.4. Increase in number of local conduction block during premature activation in hypoxia. The number of conduction blocks was calculated in three experiments, using the method demonstrated in figure 6.3. The number of conduction blocks during premature activation at different delays is plotted in panel $A$. The refractory period at the site of stimulation is given by the drawn line. During control, the maximal number of conduction blocks during earliest premature activation was 10 (panel A). In hypoxia, a hatched area is drawn encompassing those premature activations that produced more conduction blocks than during control. Immediately after the introduction of hypoxia there is an increase in the number of conduction blocks during premature activations. This increase in local conduction blocks was present during the whole period of hypoxia. 
since been studied by several other authors (Clerc 1976, Spach et al. 1982, 1986a, Paes de Carvalho 1982).

It is important to distinguish inhomogeneity in conduction caused by dispersion in refractoriness and inhomogeneity in conduction based on other local differences in conduction properties. In the case of a stable constant rhythm of $2 \mathrm{~Hz}$, after each activation sufficient time is available for the tissue to completely recover its excitability before the next activation occurs. In this situation dispersion in refractoriness has no influence on the conduction of

\section{COUPLING}

\section{INTERVAL}

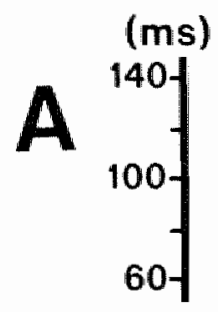

(B)

(1) 28 (1)

(a)

(12)

(8) 32112

(2) 12

(21)

$2800+28$
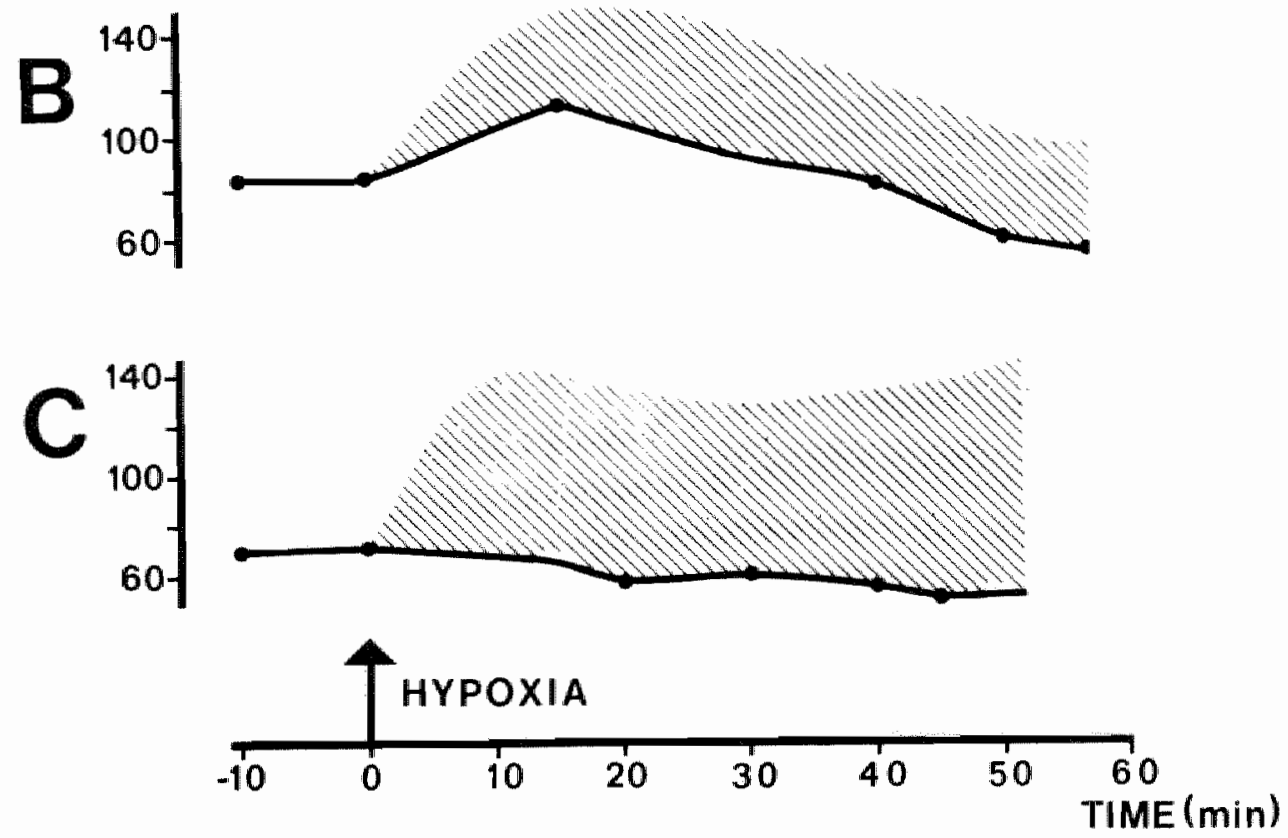
the impulse. The conduction is influenced by other factors such as the geometry of the tissue (Clerc 1976, Spach et al. 1981), by the spatial distribution in excitability or in passive electrical properties (Spach et al. 1986b). We have occasionally measured the occurrence of conduction blocks during slow pacing and found a slight increase during hypoxia. This is indicative of some increase in conduction inhomogeneity apart from inhomogeneity caused by differences in refractoriness. 


\section{Chapter VII}

\section{QUANTIFICATION OF TEMPORAL AND SPATIAL INHOMOGENEITY OF CARDIAC EXCITATION.}

\subsection{Introduction}

For the initiation of a reentrant arrhythmia the induction of unidirectional conduction block is a first prerequisite. The occurrence, of a temporary conduction block was already recognized in the first studies on circus movement (Mines 1913, 1914, Garrey 1914) and subsequently extensively confirmed (Cranefield et al. 1971a, 1971b, Wit et al. 1972a, 1972b, Allessie et al. 1976, Boineau et al. 1980, El-Sherif et al. 1977). Lewis et al. (1921) suggested that spatial differences in refractoriness may cause an irregular activation of early premature impulses leading to the induction of arcs of conduction block. Dispersion in excitability has been shown to exist in cardiac tissue and to be influenced by heart rate and the autonomic nervous system (Alessi et al. 1958, Han et al. 1964, 1966a, Janse et al. 1969). Other studies have demonstrated that spatial differences in refractoriness may coincide with the site of unidirectional conduction block and intra-myocardial reentry (Allessie et al. 1976, Boineau et al. 1980).

Recent studies have emphasized that heterogeneity in the structural properties of the myocardium may also cause local conduction block. Both atrial and ventricular tissue exhibit strong anisotropic properties, causing the impulse to propagate more rapidly longitudinally to the fiber orientation than in a transverse direction (Clerc 1976, Spach et al. 1981). Spach et al. (1982) postulated that conduction of early premature impulses would more readily block in a longitudinal than in a transverse direction.

In contrast to the recognized significance of irregularities in propagation for the initiation of reentrant arrhythmias, only a few studies have been done to measure the degree of inhomogeneity in conduction. Attempts to quantify inhomogeneity have been confined to a few recording sites (Han et al. 1966a, Merx et al. 1977, Shimizu et al. 1979). In recent years, new techniques have been developed allowing high-resolution mapping of the sequence of activation based on simultaneous recordings of a large number of electrograms (Smith et al. 1980, Wit et al. 1982, Allessie et al. 
1984, Witkowski et al. 1984).

In this chapter we present a new method to localize and quantify inhomogeneity in conduction. Using the data from high-density activation maps, the maximal local differences in activation time (maximal phase difference) were used to visualize spatial inhomogeneity in conduction (phase-maps) and to calculate an overall index of inhomogeneity. In the isolated rabbit atrium, the effects of pacing at different rates and at different sites were evaluated. In addition inhomogeneity in conduction was quantified during the application of single and multiple premature stimuli. It was found that both an increase in pacing rate and a shortening of prematurity of premature beats clearly increased the degree of inhomogeneity in conduction. Activation maps of the initiation of reentry showed that the occurrence of reentry was related to a high degree of local inhomogeneity. The potential sites of reentry could be adequately detected by phase mapping.

\subsection{Calculation of phase maps}

Figure 7.1 illustrates the procedure to quantify spatial inhomogeneity in conduction. In panel $A$ the spread of activation during regular pacing with a cycle length of $500 \mathrm{~ms}$ is shown. The stimulus was applied in the upper left corner of the preparation and the impulse propagated from the upper left to the lower right. Panels $B$ and $C$ show how, on the basis of the local activation times, a "phase map" is constructed. For all neighboring recording points, the local phase differences in activation times are calculated (panel B). For each quadruplet of electrodes, the largest phase difference is taken and plotted together as a phase map (panel C). Phase differences equal to or higher than $10 \mathrm{~ms}$ are delineated in the map. The population of all maximal phase differences is also plotted in a histogram (panel D). Percentile scores were used to describe the phase histogram. The median (P50) is a measure of the

Figure 7.1. Phase mapping as a method to quantify inhomogeneities in conduction. Panel $A$ shows the spread of activation in an isolated left atrium stimulated at the upper left corner. Local activation times are measured with a spatial resolution of $1.4 \mathrm{~mm}$. Isochrones are drawn to visualize the sequence of excitation. In panel $B$, the calculation of local phase differences is shown. For each quadruplet of recordings the differences in activation times are measured and the greatest value is plotted as the local maximal 
1 activation MaP

\section{PHASE DIFFERENCE}
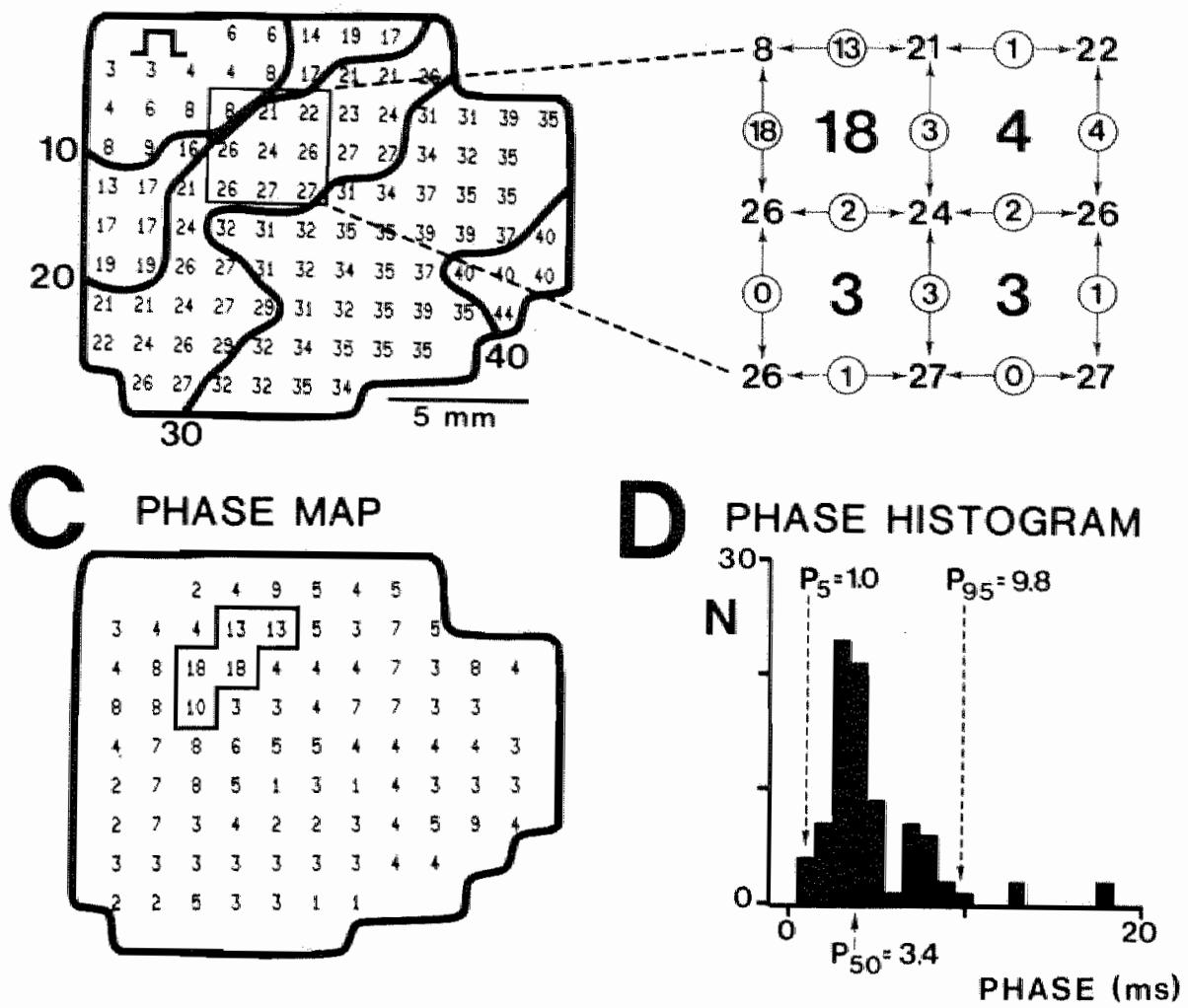

INHOMOGENEITY INDEX

$\begin{array}{rlll}\text { MEDIAN (P50) } & 3.4 \mathrm{~ms} & (2.4 \mathrm{~ms} / \mathrm{mm}) \\ \text { ABSOLUTE (P5-95) } & 8.8 \mathrm{~ms} & (6.3 \mathrm{~ms} / \mathrm{mm}) \\ \text { RELATIVE (P5-95/P50) } & 2.6 & & \end{array}$

phase difference in that area. Panel $C$ shows a map of all maximal phase differences representing the spatial inhomogeneities in conduction. In panel $D$ the phase differences are plotted as a histogram. From this histogram the median (P50), the absolute inhomogeneity in conduction (P5-95) and the relative inhomogeneity (P5-P95/P50) are calculated. 
average maximal local conduction delay in the preparation. The P5-95, expressing the spread in maximal differences in activation times, is taken as an absolute inhomogeneity index. An increase in the absolute inhomogeneity index may be caused either by an overall increase in activation times, due to slowing in conduction, or by an increase in local inhomogeneity in conduction. A relative inhomogeneity index (P5-95/P50) was used to express the inhomogeneity in conduction, independent of conduction velocity. In the data presented in this study both the absolute and the relative inhomogeneity index are used. To facilitate comparison of phase maps recorded with different spatial resolution, the inhomogeneity indices are normalized to a unit distance of $1 \mathrm{~mm}$. Statistical comparison of different groups was performed by Student's t-test and when appropriate by a paired Student's t-test.

\subsection{Direction of propagation and inhomogeneity in conduction.}

In 7 experiments, the influence of the direction of impulse propagation on inhomogeneity in conduction was investigated by pacing at various sites. Figure 7.2 gives an example of an isolated left atrium which was paced at three different sites at a cycle length of $500 \mathrm{~ms}$. Panel A shows the three activation maps. Stimulating the upper left corner, the direction of propagation was predominantly from the upper left to the lower right. Pacing at the middlle left resulted in an activation wave propagating from left to right, whereas pacing at the lower left corner started an impulse travelling to the upper right. The average speed of conduction was clearly different and varied from $35 \mathrm{~cm} / \mathrm{s}$ (pacing from the middle left) to $47 \mathrm{~cm} / \mathrm{s}$ (lower left). As can be seen from the varying distances between the isochrones, already during this slow regular

Figure 7.2. The effects of different pacing sites on inhomogeneity in conduction. Activation maps (panel A), phase maps (B) and phase histograms (C) during slow regular pacing $(2 \mathrm{~Hz})$ at three different locations. In the phase maps, areas with local differences in conduction times of $10 \mathrm{~ms}$ or more are outlined. As can be seen from the phase maps, the site of stimulation markedly influenced the resulting inhomogeneity in conduction. The absolute inhomogeneity in conduction varied from $7.2 \mathrm{~ms} / \mathrm{mm}$ during pacing at the upper left corner to $9.8 \mathrm{~ms} / \mathrm{mm}$ (middle left border) and to 3.7 $\mathrm{ms} / \mathrm{mm}$ when the lower left comer was stimulated. 
EFFECT OF PACING SITE ON INHOMOGENEITY IN CONDUCTION

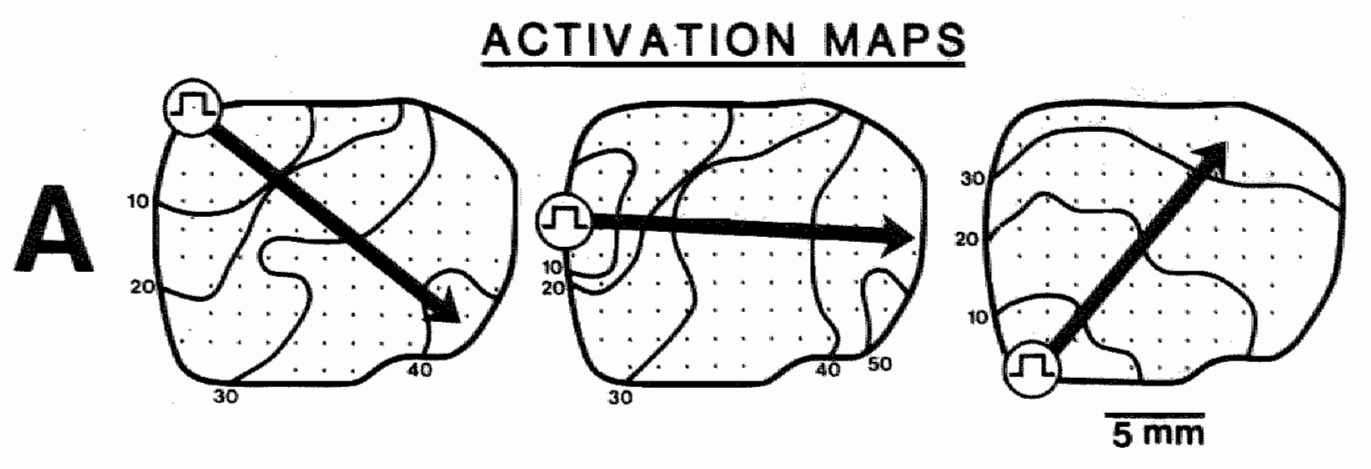

PHASE MAPS
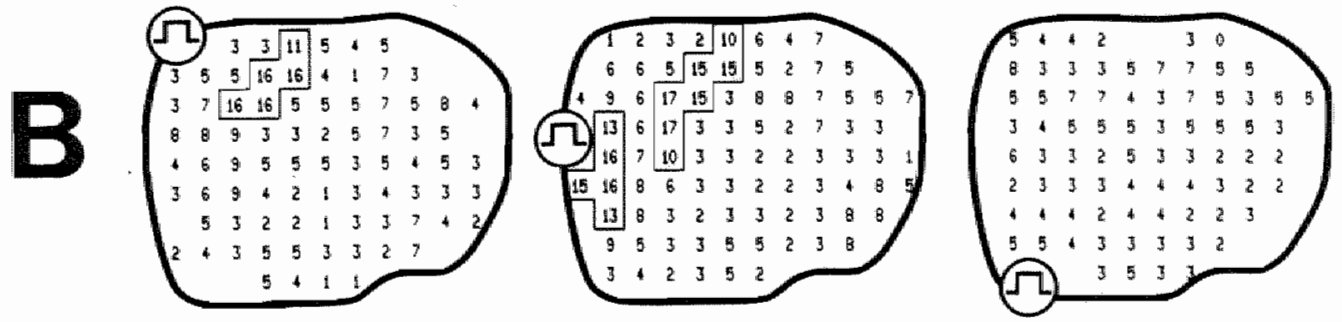

\section{PHASE HISTOGRAMS}
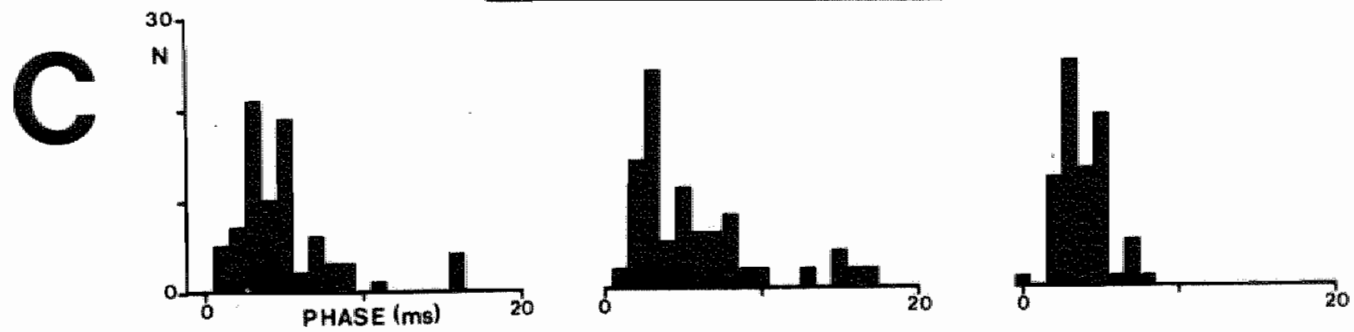

INHOMOGENEITY INDEX

$\begin{array}{lllll}\text { MEDIAN } & 2.6 & 2.9 & 2.1 \mathrm{~ms} / \mathrm{mm} \\ \text { ABSOLUTE } & 7.2 & 9.8 & 3.7 \mathrm{~ms} / \mathrm{mm} \\ \text { RELATIVE } & 2.8 & 3.4 & 1.8 & \end{array}$


rhythm (2 Hz) some degree of spatial heterogeneity in conduction existed. In panel $\mathbb{B}$, the spatial inhomogeneity in conduction is plotted as phase maps. Areas with phase differences of $10 \mathrm{~ms}$ or more are delineated. In these areas local conduction velocities of less than $14 \mathrm{~cm} / \mathrm{s}$ were present. Comparing the three phase maps, it is evident that the degree and the localization of areas of depressed conduction were dependent on the site of stimulation. When the preparation was paced from the upper left corner an area of slow conduction was present close to the site of stimulation (phase difference $16 \mathrm{~ms})$. Pacing at the middle left revealed a second area of depressed conduction in the vicinity of the new pacing site. In contrast, when the preparation was paced from the lower left corner, no conduction disturbances were detected at all. These differences in inhomogeneity in conduction are quantified by the phase histograms (panel $C$ ). The absolute inhomogeneity index waried between 3.7 and $9.8 \mathrm{~ms} / \mathrm{mm}$ while the relative inhomogeneity index varied between 1.8 and 3.4. In all seven experiments pacing sites), the absolute inhomogeneity index varied between and $9.8 \mathrm{~ms} / \mathrm{mm}$ and the relative inhomogeneity between 1.7 and 4.2 .

\subsection{The effect of prematurity on inhomogeneity in conduction.}

The spread of excitation and inhomogeneity in conduction of premature impulses were studied in 4 isolated left atria. In figure 7.3, three premature beats of increasing prematurity (coupling intervals 100,70 and $50 \mathrm{~ms}$ ) are presented. A premature impulse with a coupling interval of $100 \mathrm{~ms}$ showed an activation pattern

Figure 7.3. The effects of premature stimulation on inhomogeneity in conduction. In panel $A$ the activation maps of premature beats with a coupling interval of 100,70 and $50 \mathrm{~ms}$ are shown. The calculated phase maps (panel B) clearly demonstrate an increase in degree and extension of conduction disturbances with increasing prematurity. During the earliest premature impulse (coupling interval $50 \mathrm{~ms}$ ) local phase differences up to $40 \mathrm{~ms}$ were found. The effect of prematurity on inhomogeneity in conduction can also be seen in the phase histograms (panel C). Shortening of the coupling interval led to an increase in the median phase difference from 2.9 to $3.9 \mathrm{~ms} / \mathrm{mm}$. The absolute inhomogeneity index (P5-95) increased from $6.3 \mathrm{~ms} / \mathrm{mm}$ to $17.7 \mathrm{~ms} / \mathrm{mm}$ and the relative inhomogeneity (P5-95/50) increased from 2.2 to 4.5 . 


\section{PREMATURE ACTIVATIONS}
(100 msec)
(70 msec)
(50 msec)

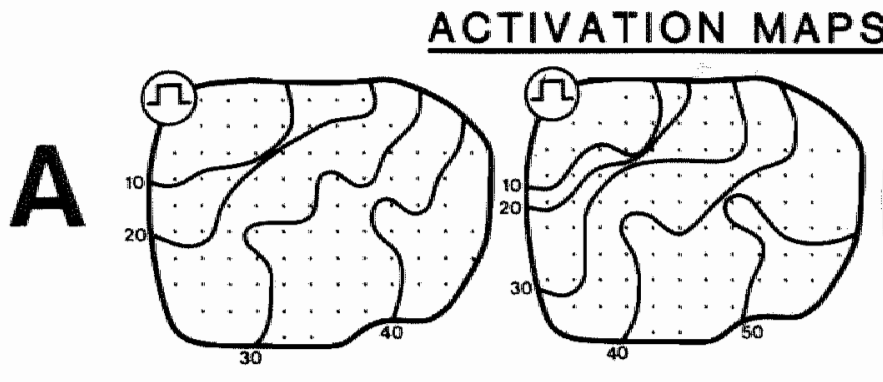

PHASE MAPS
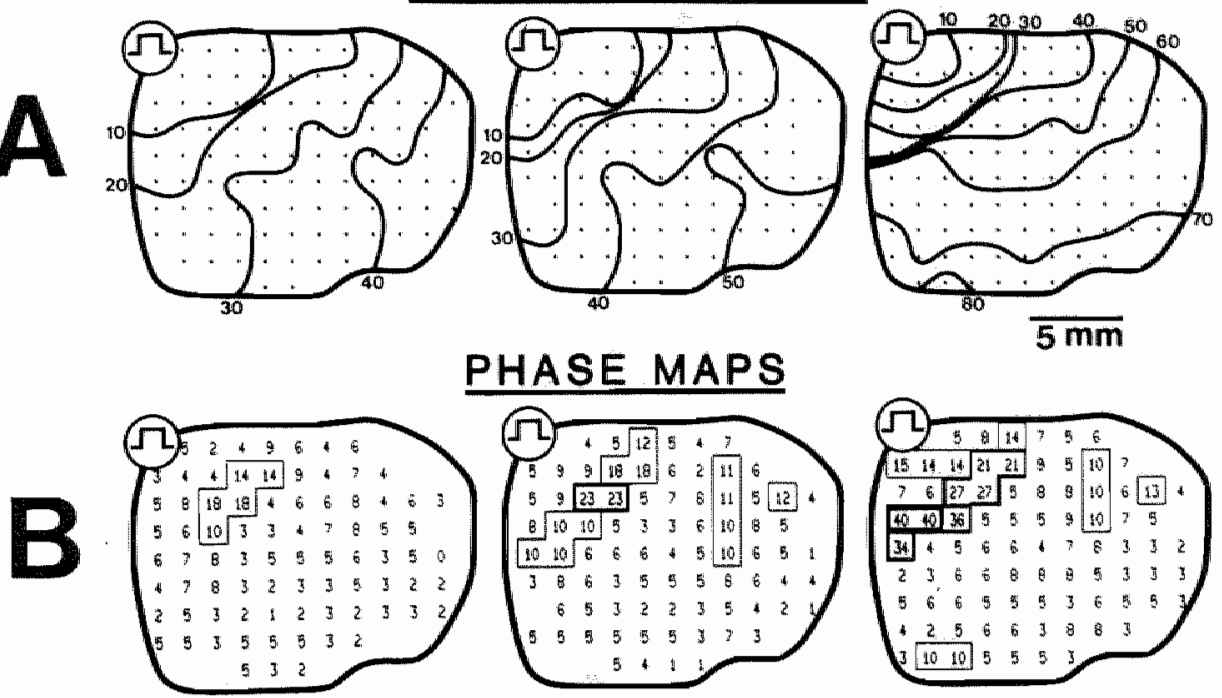

PHASE HISTOGRAMS
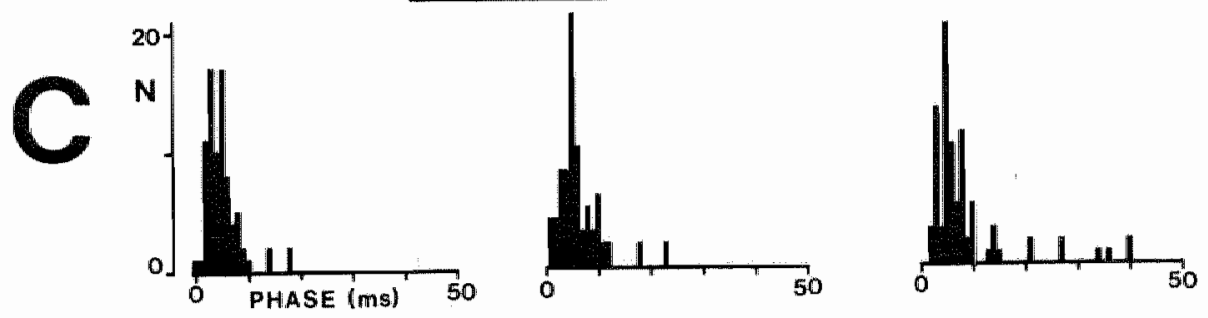

INHOMOGENEITY INDEX

$\begin{array}{llrr}\text { MEDIAN } & 2.9 & 3.4 & 3.9 \mathrm{~ms} / \mathrm{mm} \\ \text { ABSOLUTE } & 6.3 & 7.6 & 17.7 \mathrm{~ms} / \mathrm{mm} \\ \text { RELATIVE } & 2.2 & 2.2 & 4.5\end{array}$


similar to the propagation during basic rhythm (compare figure 7.2). Decreasing the coupling interval from 100 to 70 and $50 \mathrm{~ms}$ resulted in progressive slowing of propagation, the total activation time of the preparation increasing from 40 to 50 and $80 \mathrm{~ms}$. The phase maps (panel B) showed a clear increase in both degree and area of depressed conduction. Decrease in the coupling interval induced an extension of the outlined areas with phase differences greater than 10 ms. During the propagation of the earliest premature impulse (coupling interval $50 \mathrm{~ms}$ ) local phase differences as large as $40 \mathrm{~ms}$ were found. The median phase difference (panel $C$ ) increased from 2.9 to 3.4 to $3.9 \mathrm{~ms} / \mathrm{mm}$ and the absolute inhomogeneity index from 6.3 to 7.6 to $17.7 \mathrm{~ms} / \mathrm{mm}$. The relative inhomogeneities of premature impulses with a coupling interval of 100 and $70 \mathrm{~ms}$ were identical (2.2), implicating that the change in the absolute inhomogeneity index was caused by a general increase in local phase differences. Shortening of the coupling interval to $50 \mathrm{~ms}$ caused a marked increase in the relative inhomogeneity index from 2.2 to 4.5 expressing an increase in conduction inhomogeneity of more than $100 \%$.

In figure 7.4 the effects of early premature beats on the inhomogeneity parameters are plotted for 18 pacing sites. The median phase differences and the absolute and relative inhomogeneity indices measured during basic rhythm and the earliest possible premature beat are plotted. During early premature beats all these parameters were significantly increased $(\mathrm{p}<0.001)$ compared to basic rhythm. The median increased from an average of 2.8 $\mathrm{ms} / \mathrm{mm} \pm 0.7$ during basic rhythm to $3.7 \mathrm{~ms} / \mathrm{mm} \pm 0.7$ during the earliest premature impulses. The absolute inhomogeneity index increased from $6.5 \pm 2.1$ to $11.4 \pm 3.4 \mathrm{~ms} / \mathrm{mm}$ and the relative inhomogeneity increased from $2.3 \pm 0.6$ to $3.1 \pm 0.8$.

To study whether the inhomogeneity is further increased with multiple premature beats, three successive premature stimuli (S2-S4) were applied after a series of 20 basic stimuli (S1). The delay of each premature stimulus was set at the shortest possible coupling interval at which a premature response occurred. Phase maps and phase histograms of the three successive premature impulses were made and the absolute and relative inhomogeneity in conduction were calculated. In figure 7.5 the mean values of 9 experiments are given. The absolute inhomogeneity increased from $5.4 \mathrm{~ms} / \mathrm{mm}$ during basic rhythm (S1) to $10.6 \mathrm{~ms} / \mathrm{mm}$ during the first premature beat (S2; $\mathrm{p}<0.001$ ), the relative inhomogeneity increasing from 2.2 to 3.1 $(\mathrm{p}<0.01)$. A second premature beat (S3) further significantly increased the absolute and relative inhomogeneities to $13.3 \mathrm{~ms} / \mathrm{mm}$ 


\section{PREMATURE ACTIVATION AND \\ INHOMOGENEITY IN CONDUCTION}
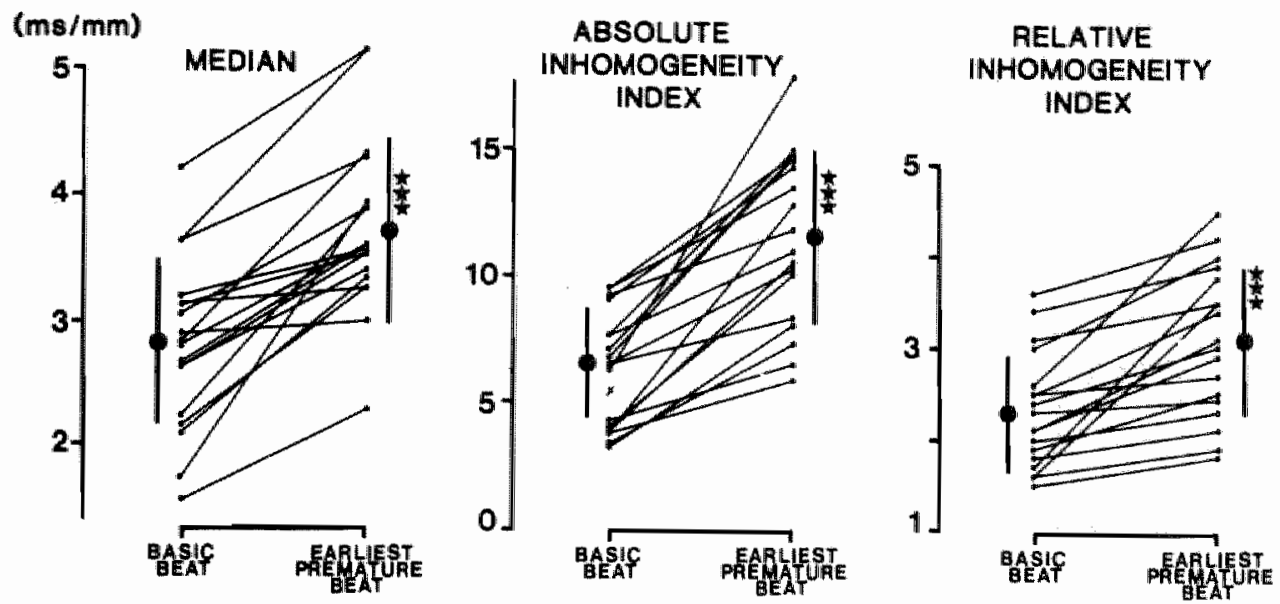

Figure 7.4. The effects of premature activation on inhomogeneity in conduction. Individual values of 18 different measurements are plotted together with the mean and standard deviation (*** = $p<0.001 ;$ paired student $t$-test). Premature activation increased the median phase difference from 2.8 to $3.7 \mathrm{~ms} / \mathrm{mm} \quad(p<0.001)$. Both the absolute inhomogeneity and the relative inhomogeneity also increased upon earliest premature stimulation. The absolute inhomogeneity index increased from 6.5 to $11.4 \mathrm{~ms} / \mathrm{mm} \quad(p<0.001)$ and the relative inhomogeneity index from 2.3 to $3.1 \quad(p<0.001)$.

$(\mathrm{p}<0.01)$ and $3.6 \quad(\mathrm{p}<0.05)$. The third successive premature beat (S4) did not enhance the heterogeneity in conduction any further. The cumulative effect of multiple premature stimuli resulted in an increase in the absolute inhomogeneity index from 5.4 to 13.3 $\mathrm{ms} / \mathrm{mm}$ and of the relative inhomogeneity index from 2.2 to 3.4 .

\subsection{Pacing rate and inhomogeneity in conduction.}

The influence of pacing rate on inhomogeneity in conduction was studied in three left atrial preparations. In figure 7.6 the analysis of the excitation at a driving rate of 5,7 and $10 \mathrm{~Hz}$ are shown. Increase of the pacing rate induced a progressive decrease in 


\section{EFFECT OF MULTIPLE PREMATURE BEATS ON INHOMOGENEITY IN CONDUCTION}

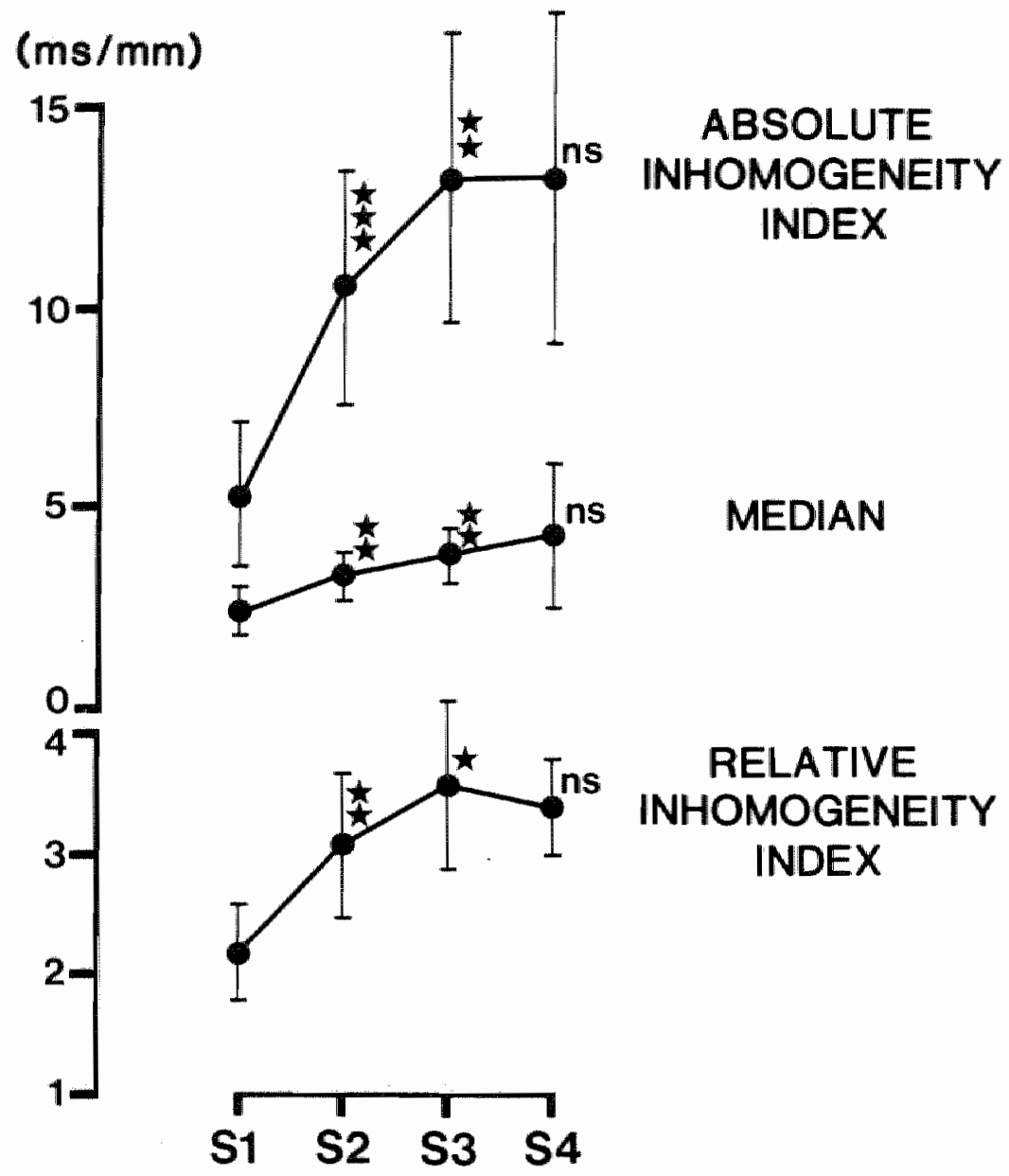

Figure 7.5. The influence of multiple premature beats on inhomogeneity in conduction. The absolute inhomogeneity index, the median of local phase differences and the relative inhomogeneity index are plotted as measured during basic rhythm (S1) and during three successive earliest premature impulses (S2, S3, S4). The plotted values are the mean values $\pm S D$ of 9 different experiments. A paired student t-test was used to compare each beat with 
conduction velocity, the total activation time increasing from 50 to 60 and $110 \mathrm{~ms}$ respectively (panel A). The activation map of $10 \mathrm{~Hz}$ reveals a clear arc of conduction block of about $7 \mathrm{~mm}$ in length from halfway the $20 \mathrm{~ms}$ isochrone to the upper border of the preparation. The phase maps emphasize both the location and degree of conduction disturbances during incremental pacing. At higher pacing rates the number of areas with local phase differences greater than $10 \mathrm{~ms}$ increased markedly.

Along the line of conduction block present during pacing at 10 $\mathrm{Hz}$ the local phase differences gradually increased from $23 \mathrm{~ms}$ at one end of the arc of block to $74 \mathrm{~ms}$ at the border of the preparation. These large differences in local activation times were caused by a change in direction of the propagating wavefront around the arc of conduction block. The area proximal to the line of block was activated simultaneously at $20 \mathrm{~ms}$ whereas the area distal to the line of block was activated sequentially by the depolarization wave turning around one end of the arc of block.

The conduction disturbances induced by pacing at increasing frequencies are quantified by the phase histograms in panel $C$. The absolute inhomogeneity index increased from $8.4 \mathrm{~ms} / \mathrm{mm}$ at a pacing rate of $5 \mathrm{~Hz}$ to $13.7 \mathrm{~ms} / \mathrm{mm}$ at $7 \mathrm{~Hz}$ and $25.5 \mathrm{~ms} / \mathrm{mm}$ at $10 \mathrm{~Hz}$. Likewise, the relative inhomogeneity increased from 2.8 to 4.0 and 5.0 respectively.

Large differences in activation times may indicate potential sites for reentry. In the phase map obtained at a pacing rate of $10 \mathrm{~Hz} \mathrm{a}$ maximal phase difference of $74 \mathrm{~ms}$ was found near the upper border of the preparation. The refractory period in that area was in the order of $80 \mathrm{~ms}$. Thus only a few milliseconds were left between the delayed arrival of the impulse and restoration of excitability of the fibers proximal to the line of block. This prevented the delayed excitation wave from reentering the previously activated tissue. A slight shortening of the refractory period or a small increase in the arrival time of the impulse would have led to reentry. Indeed, series of reentrant excitation were sometimes observed after premature stimulation or a burst of rapid pacing (see figure 7.9).

preceding beat. While the first and the second premature impulses (S2 and S3) significantly increased inhomogeneity in propagation, a third premature beat did not further enhance the degree of inhomogeneity in conduction ( $n s=$ not significant; $*=p<0.05 ;{ }^{* *}=$ $p<0.01 ; * * *=p<0.001)$. 
PACING RATE
$(5 \mathrm{~Hz})$
$(7 \mathrm{~Hz})$
$(10 \mathrm{~Hz})$

\section{ACTIVATION MAPS}

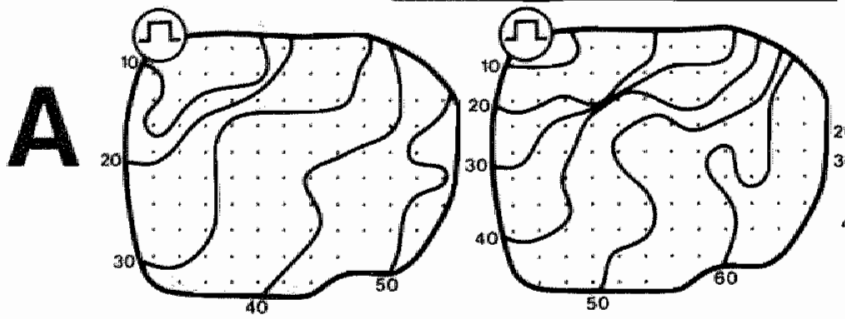

PHASE MAPS
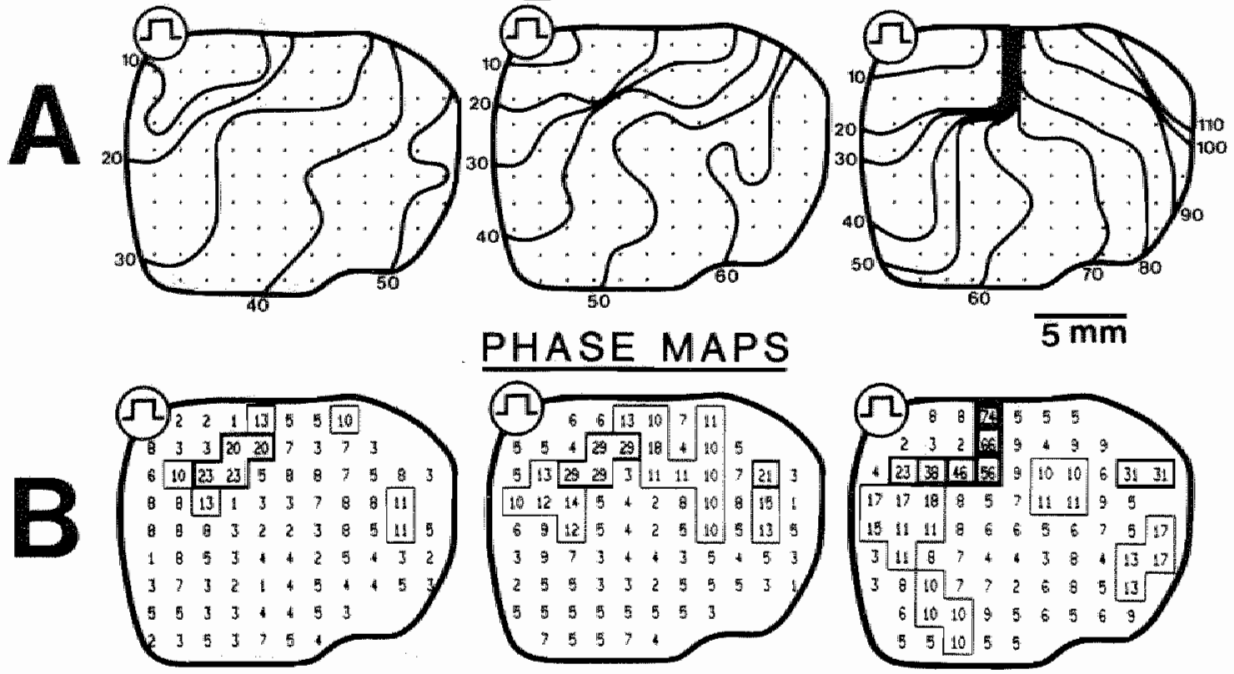

PHASE HISTOGRAMS
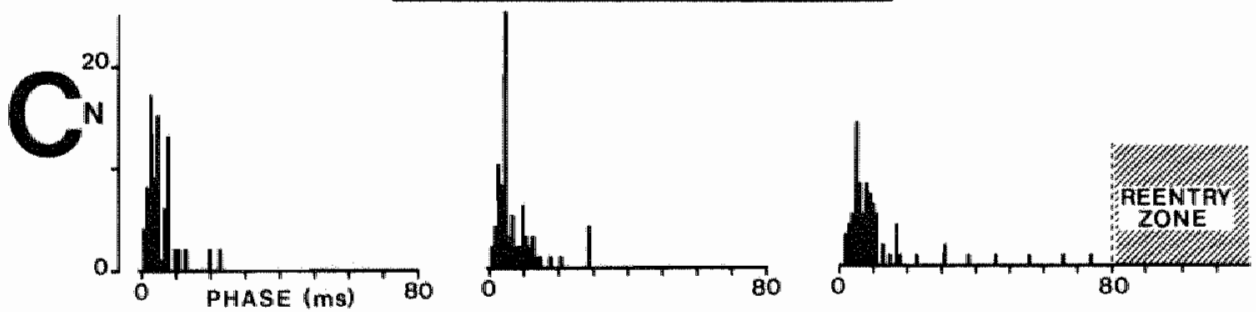

INHOMOGENEITY INDEX

$\begin{array}{llrrr}\text { MEDIAN } & 3.0 & 3.4 & 5.1 \mathrm{~ms} / \mathrm{mm} \\ \text { ABSOLUTE } & 8.4 & 13.7 & 25.5 \mathrm{~ms} / \mathrm{mm} \\ \text { RELATIVE } & 2.8 & 4.0 & 5.0 & \end{array}$


Figure 7.6. The effects of rapid pacing on inhomogeneity in conduction. In panel $A$ the activation maps during pacing at 5,7 and $10 \mathrm{~Hz}$ are given. Panel $B$ shows the corresponding phase maps demonstrating a marked increase in conduction distubances during incremental pacing. In panel $C$ the histograms of local phase differences during the three different pacing rates are given. Shortening of the pacing interval to $100 \mathrm{~ms}(10 \mathrm{~Hz})$ caused a clear increase of the median phase difference to $5.1 \mathrm{~ms} / \mathrm{mm}$. The absolute inhomogeneity increased to $25.5 \mathrm{~ms} / \mathrm{mm}$ and the relative inhomogeneity to 5.0. A reentry zone, where the phase difference exceeds the refractory period, is indicated in the phase histogram. In this example the largest phase difference of $74 \mathrm{~ms}$ during rapid pacing with $10 \mathrm{~Hz}$ was slightly less than the local refractory period ( 80 ms) and consequently no reentry occurred.

The refractory period of the tissue determines the critical phase difference at which reentry will occur and is indicated in the phase histogram as a reentry zone. The phase maps therefore not only provide an image of spatial inhomogeneities in conduction but also identify potential sites for reentry.

In figure 7.7 the effects of incremental pacing on inhomogeneity in conduction are given for all observations $(n=12)$. Changing pacing rate from 2 to $5 \mathrm{~Hz}$ slightly increased the median of phase differences whereas homogeneity in conduction was not affected. A further increase in pacing rate to 7,8 and $10 \mathrm{~Hz}$ progressively increased the median phase differences and inhomogeneity in conduction. At the highest pacing rate of $10 \mathrm{~Hz}$ the absolute inhomogeneity index attained a mean value of $36.4 \mathrm{~ms} / \mathrm{mm} \pm 19.4$ and the relative inhomogeneity $5.3 \pm 2.1$.

The detoriation in conduction during rapid pacing was much stronger than the conduction disturbances during a single premature beat. This is shown in table 7.1 in which the mean values during basic rhythm ( $2 \mathrm{~Hz}$ ), early premature beats (coupling interval $60 \pm 8$ $\mathrm{ms})$ and rapid pacing $(10 \mathrm{~Hz})$ are compared. Earliest premature activation enhanced the median phase difference from 2.8 to 3.7 $\mathrm{ms} / \mathrm{mm}$, whereas during rapid pacing the median increased to 6.6 $\mathrm{ms} / \mathrm{mm}$. The absolute inhomogeneity index was $6.5,11.4$ and 36.4 $\mathrm{ms} / \mathrm{mm}$ respectively. Likewise, the relative inhomogeneity index was more influenced by rapid pacing (5.3) than by premature activation (3.1). 


\section{RAPID PACING AND}

\section{INHOMOGENEITY IN CONDUCTION}

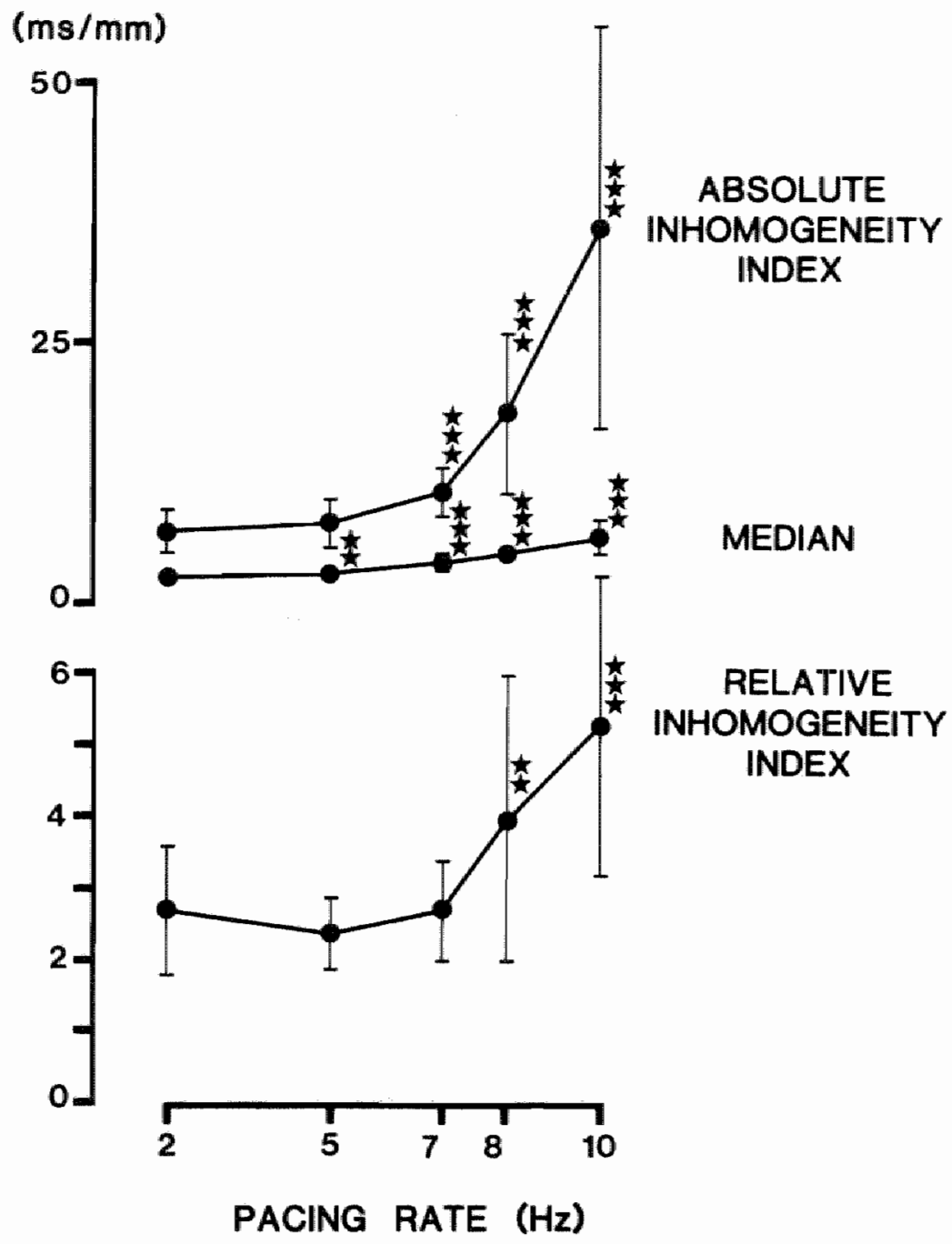

Figure 7.7. The effects of frequency of stimulation on inhomogeneity in conduction. Mean and standard deviation of the absolute and relative inhomogeneity indices and the median of local maximal phase differences are plotted as a function of the pacing frequency $(n=12)$. At high pacing rates $(>7 \mathrm{~Hz})$ inhomogeneity in conduction was strongly increased $\left.{ }^{\prime * *}=p<0.01 ; * * *=p<0.001\right)$. 
Table 7.1

THE EFFECTS OF RATE AND RHYTHM ON INHOMOGENEITY IN CONDUCTION

$\begin{array}{lcc}\begin{array}{l}\text { Basic } \\ \text { Rhythm } \\ (2 \mathrm{~Hz})\end{array} & \begin{array}{c}\text { Earliest } \\ \text { Premature } \\ \text { Beat }\end{array} & \begin{array}{l}\text { Rapid } \\ \text { Pacing } \\ (10 \mathrm{~Hz})\end{array} \\ 18 & 18 & 12\end{array}$

Median of local phase differences (ms/mm)

Absolute

Inhomogeneity

$(\mathrm{ms} / \mathrm{mm})$

Relative

Inhomogeneity

2.8

$\pm 0.7$

6.5

$\pm 2.1$

$\pm 0.6$
$3.7^{*}$

$\pm 0.7$

11.4*

$\pm 3.4$

3.1*

$\pm 0.8$
$5.3+$

$6.6+$

$\pm 1.6$

$36.4+$

$\pm 19.4$

$\pm 2.1$

* $=\mathrm{p}<0.001$ (compared to basic rhythm; paired Student t-test)

$+=p<0.001$ (compared to earliest premature beat; Student t-test)

7.6 Inhomogeneity in conduction and distance to site of stimulation.

Several reports have indicated that inhomogeneities in conduction occur more frequently near the site of stimulation, while farther away conduction becomes more homogeneous (Han et al. 1966b, Janse et al. 1969, Myerburg et al. 1973). To investigate whether conduction disturbances are related to the site of stimulation, local phase differences were plotted as a function of the distance to the stimulus electrode. The pacing protocol included the induction of two successive premature beats and rapid pacing $(10 \mathrm{~Hz})$ at four different pacing sites. In figure 7.8 the local phase differences are plotted as a function of distance from the site of stimulation as measured during basic rhythm $(2 \mathrm{~Hz})$, during the first and second premature beat and during rapid pacing (panels A-D).

During basic rhythm the phase differences ranged between 0 and 

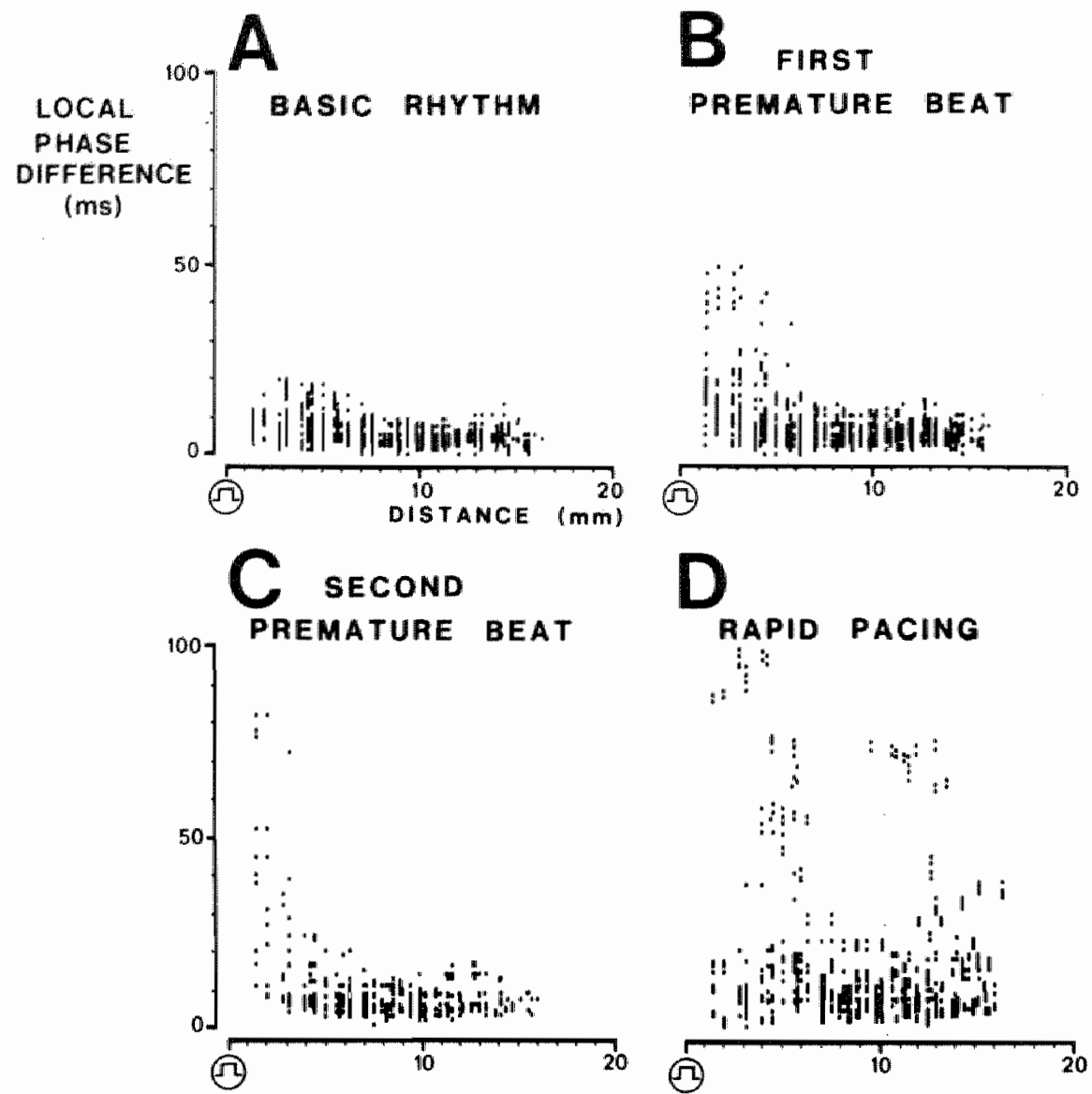

Figure 7.8. Inhomogeneity in conduction as a function of distance from the site of stimulation. A left atrial preparation was stimulated from 4 different locations during basic rhythm $(2 \mathrm{~Hz})$, single and double earliest premature beats and during rapid pacing (10 Hz). Phase maps were constructed from each beat and all the calculated local phase differences were plotted in relation to the distance from the site of stimulation. Panel $A=$ basic rhythm, panel $B=$ first premature beat, panel $C=$ second premature beat, panel $D$ = rapid pacing. During basic rhythm, local phase differences were less than $20 \mathrm{~ms}$ (panel A). Both a single and two successive premature activations induced higher phase differences in the vicinity of the site of stimulation. During rapid pacing (panel D) larger phase differences were found both close to and farther away from the site of stimulation. 
20 ms. There was no significant differences at sites close to and farther away from the stimulus electrodes. During single premature beats (panel B) the part of the preparation that was remote from the site of stimulation displayed comparable phase differences as during basic rhythm. However within an area of $6 \mathrm{~mm}$ around the stimulating electrode much higher phase differences up to $50 \mathrm{~ms}$ were found. Induction of a second premature beat (panel C) indaced even higher phase differences (up to $80 \mathrm{~ms}$ ) also located in the vicinity of the stimulus electrode. This localization of conduction disturbances around the stimulating electrodes can be explained by the differences in local cycle length during the propagation of a premature wavefront. Because the premature impulse propagates more slowly than the previous impulse, the local coupling interval between the basic and the premature impulse gets longer at longer distances from the site of stimulation.

In contrast to the conduction disturbances fond during single or double premature beats, during rapid pacing the sites of conduction disturbances were not exclusively located in the vicinity of the stimulus electrodes but occurred throughout the preparation (panel D). During constant rapid pacing all parts of the preparation are activated at the same short cycle length and potential sites of conduction block can be detected independent of the site of stimulation.

\subsection{Reentrant activity and inhomogeneity in conduction.}

Reentrant tachy-arrhythmias can be initiated by programmed electrical stimulation (Han et al. 1969, Wellens 1971). Under some conditions the application of an appropriately timed single premature stimulus may already induce reentry. In other cases, a series of two or more closely coupled premature impulses may be required to produce reentry. An example of the initiation of reentry in the isolated rabbit atrium is shown in figure 7.9. During a basic rhythm of $2 \mathrm{~Hz}$. (S1) two successive premature stimuli were given with a coupling interval of $62 \mathrm{~ms}(\mathrm{~S} 1-\mathrm{S} 2)$ and $46 \mathrm{~ms}$ (S2-S3). As can be seen from the recording at the top of figure 7.9 , this initiated a tachy-arrhythmia with a cycle length of $80 \mathrm{~ms}$.

The sequence of activation during basic rhythm was the same as shown in figure 7.2. In panels $A$ of figure 7.9, the activation maps of the first and second premature beat are given together, with the first beat of the reentrant arrhythmia. During the first premature beat two areas of conduction block were present, one close to the 


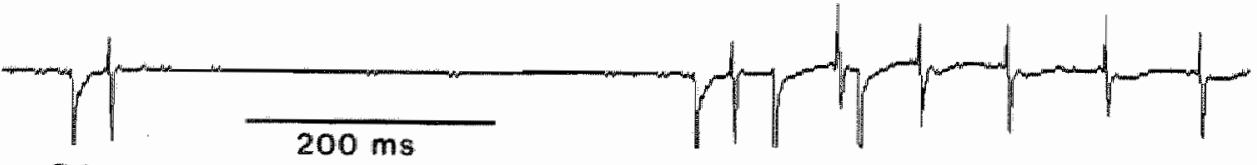

S1

S1 $\mathrm{S2} \quad \mathrm{S} 3$

FIRST

PREMATURE

BEAT

\section{SECOND}

PREMATURE

BEAT
FIRST

REENTRANT

BEAT

\section{ACTIVATION MAPS}
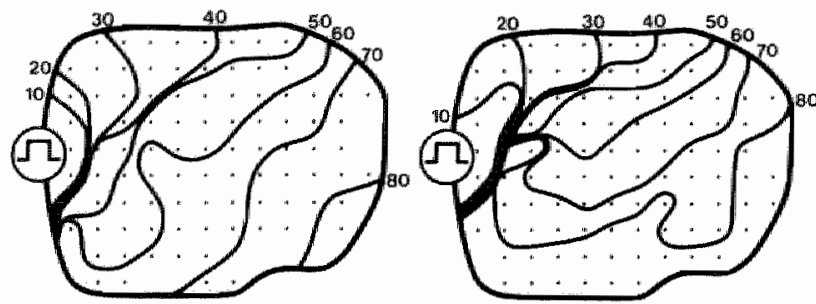

PHASE MAPS
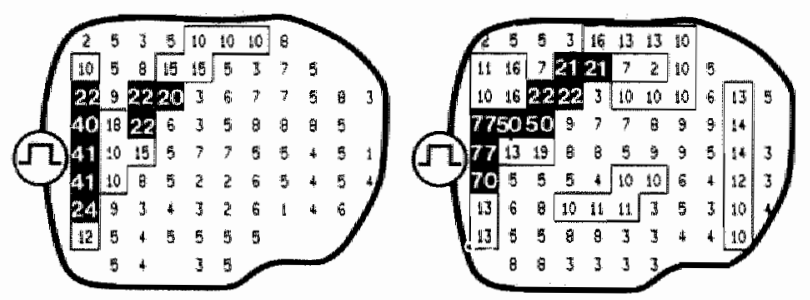

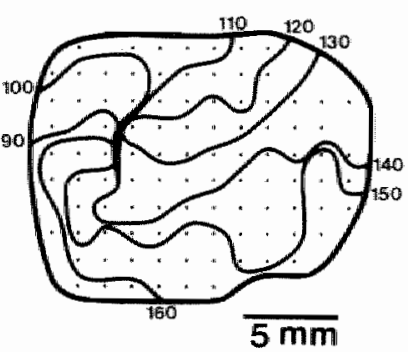

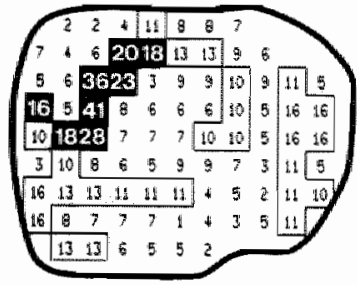

PHASE HISTOGRAMS
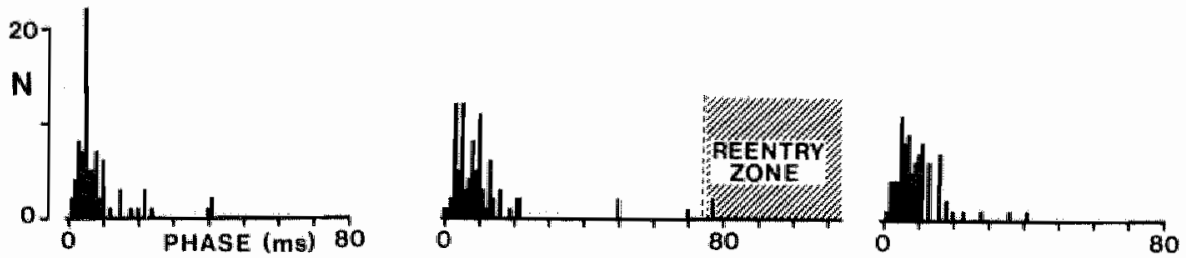

INHOMOGENEITY INDEX

$\begin{array}{lrrrr}\text { MEDIAN } & 3.5 & 5.4 & 5.4 \mathrm{~ms} / \mathrm{mm} \\ \text { ABSOLUTE } & 14.6 & 33.7 & 12.7 & \mathrm{~ms} / \mathrm{mm} \\ \text { RELATIVE } & 4.2 & 6.2 & 2.4 & \end{array}$


site of stimulation and another a few millimeters away. In the corresponding phase maps (panel B) these two areas are indicated by solid squares.

During the second premature beat inhomogeneity in conduction increased. The two separate areas of conduction block has been united into a single arc of block of about $7 \mathrm{~mm}$ long. As can be seen from the activation map of the second premature beat, the impulse was first conducted along the left side of this line of block towards the upper part of the preparation. It then rotated clockwise around the upper end of the line of block to propagate downwards along the right side of the line of block.

As shown by the local phase differences close to the site of stimulation, the impulse returned to the site of origin after $77 \mathrm{~ms}$. This local delay in activation was sufficiently long for local excitability to recover and reexcitation to occur. This is demonstrated in the activation map of the first reentrant beat and the wavefront continued to propagate in a clockwise direction. The phase map of the first revolution depicts the location of the central arc of conduction block around which the impulse rotated and is outlined by a continuous line of phase differences ranging between 16 and $41 \mathrm{~ms}$.

Figure 7.10 depicts the changes in inhomogeneity in conduction during the initiation and the perpetuation of the tachy-arrhythmia. The application of two closely coupled stimuli increased the median phase difference from 2.9 to 3.5 to $5.4 \mathrm{~ms} / \mathrm{mm}$ and enhanced the absolute inhomogeneity index from 9.8 to 14.6 to $33.7 \mathrm{~ms} / \mathrm{mm}$. "These changes resulted in an increase of the relative inhomogeneity from 3.4 to 4.2 to 6.2 . During the resulting reentrant arrhythmia,

Figure 7.9. Inthomogeneity in conduction during the induction of a reentrant arrhythmia. The application of two early premature beats (S2 and S3) following a basic beat (S1) initiated a tachy-arrhythmia as shown in the electrogram at the top of the figure. The activation maps of the two premature beats and ofthe first reentrant beat are given in panel $A$. During propagation of the second premature beat an arc of conduction block occurred which forced the impulse to revolve around this arc in a clockwise direction. Reentry occurred in the area proximal to the line of block. The corresponding phase maps quantify the increase in conduction inhomogeneity by the two premature beats. Along the line of block a maximal phase difference of $77 \mathrm{~ms}$ was measured, large enough for local excitability to restore and reentry to accur. 
A
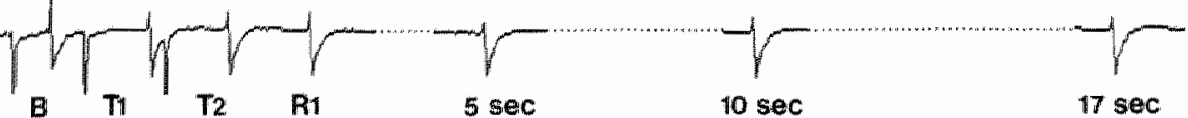

$10 \mathrm{sec}$

17 sec

(ms)

$\underbrace{110-1}$

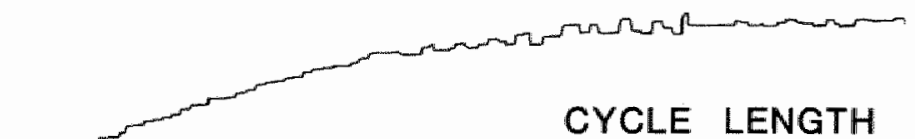

(ms/mm)

$30-1$

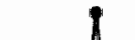

ABSOLUTE

INHOMOGENEITY

INDEX

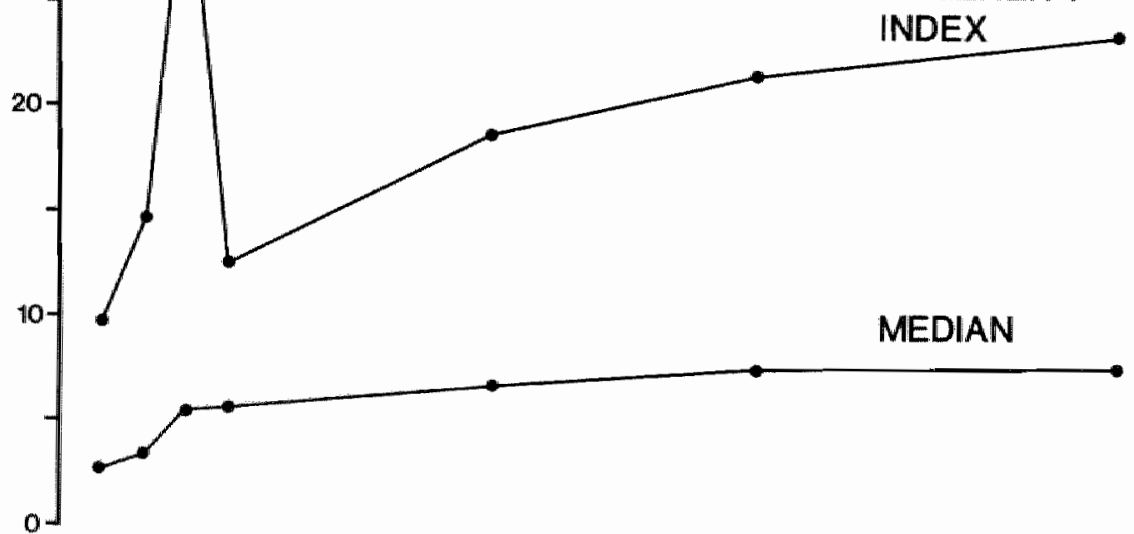

-1

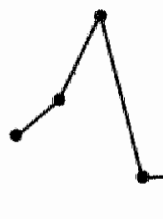

RELATIVE

INHOMOGENEITY

INDEX
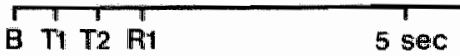

$10 \sec$

$17 \mathrm{sec}$ 
Figure 7.10. Changes in inhomogeneity in conduction during the initiation and perperuation of a reentrant arrhythmia. The tachyarrhythmia shown in the upper panel was initiated by two closely coupled premature stimuli (TI and T2). In panel $B$, the beat-tobeat cycle length of the tachy-arthythmia is plotted. The inhomogeneity indices reach maximal values during the initiation of the arrhythmia. See text for further description.

the median phase difference continued to increase to reach a stable vallue of $7.4 \mathrm{~ms} / \mathrm{mm}$. The absolute and relative inhomogeneity indices were maximal during the second induced premature beat which led to reentry. After the initiation of reentry both the absolute and relative inhomogeneity dramatically decreased again. During the perpetuation of the arrhythmia these indices gradually increased to reach values comparable to regular pacing at that rate.

\subsection{Hypoxia and inhomogeneity in conduction.}

As was shown in chapters $V$ and VI, tachy-arrhythmias in hypoxia are caused by a marked increase in the occurrence of local conduction block. We have investigated whether the method of phase mapping is able to detect and quantify the conduction disturbances induced by hypoxia. In figure 7.11 a premature beat, with a coupling interval of $85 \mathrm{~ms}$, was induced both during control and after 65 minutes in hypoxia. During control, this was the earliest premature impulse that could be induced while in hypoxia the refractory period had decreased from $85 \mathrm{~ms}$ to $64 \mathrm{~ms}$. The premature impulse induced at this earliest coupling interval is also plotted in figure 7.11.

During control, the earliest premature stimulus induced an impulse that was conducted with few delays in the preparation. In hypoxia, a premature activation, initiated at the same coupling interval, revealed an arc of conduction block close to the site of stimulation. Premature stimulation at the shortest coupling interval in hypoxia, further lengthened the arc of conduction block which now reached the lower border of the preparation.

The increase in conduction inhomogeneity in bypoxia is presented in the corresponding phase maps (panel B). During control the earliest premature impulse induced two discrete areas of conduction disturbances (phase differences 20-30 ms). In hypoxia, premature activation at the same coupling interval induced an increase in 


\section{PREMATURE ACTIVATIONS}

$\begin{array}{lll}\text { CONTROL } & \text { HYPOXIA } & \text { HYPOXIA } \\ (85 \mathrm{~ms}) & (85 \mathrm{~ms}) & (64 \mathrm{~ms})\end{array}$

\section{ACTIVATION MAPS}

A
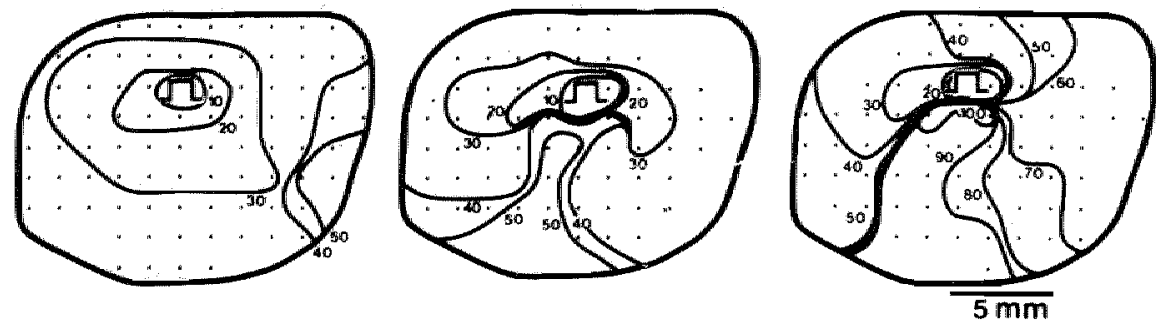

PHASE MAPS

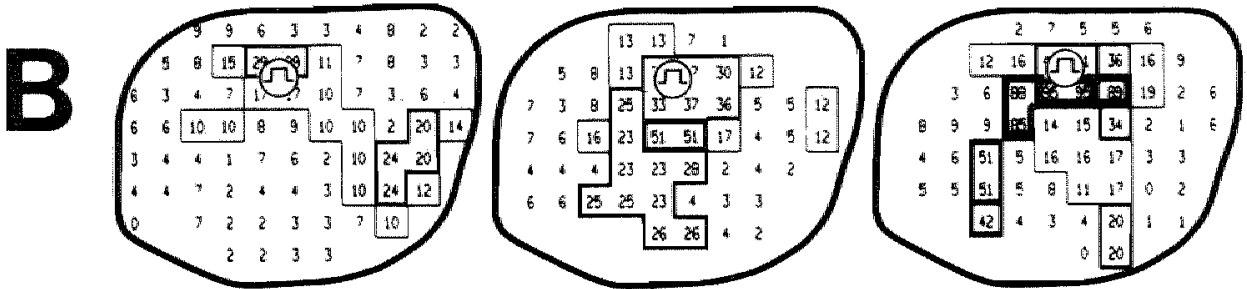

PHASE HISTOGRAMS
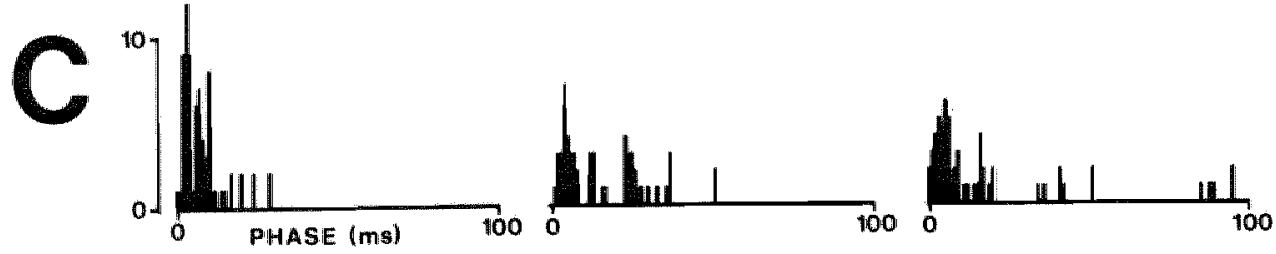

INHOMOGENEITY INDEX

MEDIAN

4.0

ABSOLUTE 15.7

RELATIVE $\quad 3.9$
5.7

25.2

4.4
$5.4 \mathrm{~ms} / \mathrm{mm}$

$62.7 \mathrm{~ms} / \mathrm{mm}$

11.6 
Figure 7.11. The effects of hypoxia on inhomogeneity in conduction. In panel $A$ activation maps are drawn of the earliest premature beat during control (coupling interval $85 \mathrm{~ms}$ ) and of two premature beats during hypoxia; the first at the same coupling interval as during control and the second at the shortest possible coupling interval (64 ms). During hypoxia, an arc of conduction block was induced below the site of stimulation which reached the lower left edge of the preparation upon premature stimulation at the shortest coupling interval. The phase maps in panel $B$ visualize the spatial increase in conduction inhomogeneities caused by hypoxia. The corresponding phase histograms demonstrate that hypoxia increased the absolute inhomogeneity index from 15.7 to $62.7 \mathrm{~ms} / \mathrm{mm}$ and the relative inhomogeneity from 3.9 to 11.6 .

degree and extension of inhomogeneities in conduction, the greatest local phase difference being $51 \mathrm{~ms}$. During the earliest premature activation (coupling interval $64 \mathrm{~ms}$ ) the area of conduction block was further extended and a greatest phase difference of $95 \mathrm{~ms}$ was measured. In this example the absolute inhomogeneity index of the earliest premature impulse increased from $15.7 \mathrm{~ms} / \mathrm{mm}$ during control to $62.7 \mathrm{~ms} / \mathrm{mm}$ during hypoxia and the relative inhomogeneity increased from 3.9 to 11.6 .

The effects of hypoxia on - inhomogeneity in conduction were quantified both during regular rhythm $(2 \mathrm{~Hz})$ and during the earliest premature activation in 19 experiments. The mean values of the conduction parameters measured during control (empty bars) and during hypoxia (filled bars) are plotted in figure 7.12. At a basic rhythm of $2 \mathrm{~Hz}$, hypoxia increased the median phase difference from 2.8 to $4.0 \mathrm{~ms} / \mathrm{mm}$, enhanced the absolute inhomogeneity index from 6.5 to $13.0 \mathrm{~ms} / \mathrm{mm}$ and augmented the relative index from 2.3 to 3.4. During early premature beats the median phase difference increased from $3.7 \mathrm{~ms} / \mathrm{mm}$ during control to $6.9 \mathrm{~ms} / \mathrm{mm}$ in hypoxia. The absolute inhomogeneity index increased from 11.4 to $31.2 \mathrm{~ms} / \mathrm{mm}$ and the relative inhomogeneity from 3.1 to 4.7 .

\subsection{Discussion}

In this chapter, a method to visualize and quantify inhomogeneity in conduction is described. Local inhomogeneity in conduction was evaluated by calculating the greatest difference in activation times between adjacent recording sites. These differences in activation 


\section{THE EFFECTS OF HYPOXIA ON}

\section{INHOMOGENEITY IN CONDUCTION}

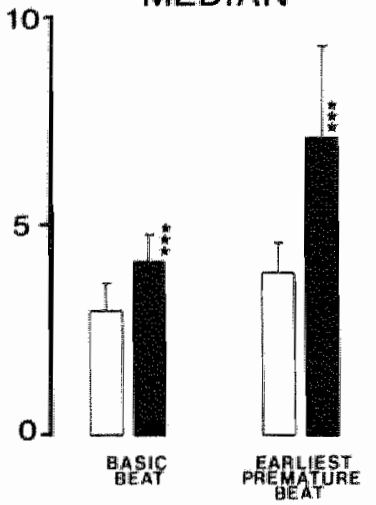

ABSOLUTE INHOMOGENEITY

INDEX

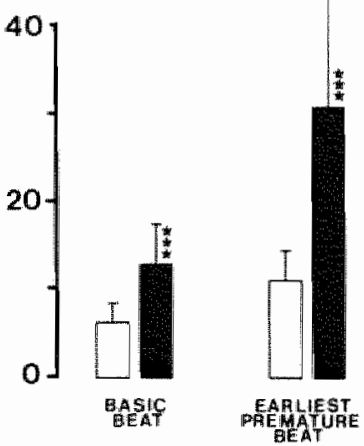

RELATIVE INHOMOGENEITY INDEX

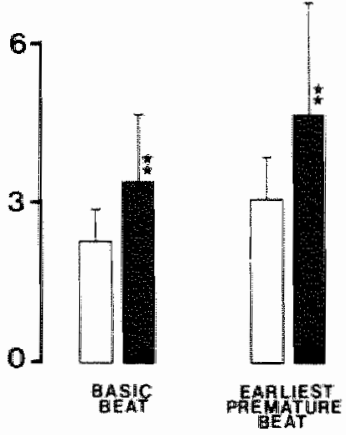

Figure 7.12. The effects of hypaxia on inhomogeneity in conduction as measured in 5 left atrial preparations $(N=19)$ during basic rhythm and during earliest premature activation. The medians of maximal phase difference (left panel), the absolute inhomogeneity indices (middle panel) and the relative inhomogeneity indices (right panel) were quantified during control (empty bars) and during hypoxia (filled bars). During basic rhythm, hypoxia increased the median phase differences from 2.8 to $4.0 \mathrm{~ms} / \mathrm{mm}$ and during premature activation from 3.7 to $6.9 \mathrm{~ms} / \mathrm{mm}$. Hypoxia also increased significanthy the occurrence of conduction disturbances. The absolute inhomogeneity index increased during basic pacing from 6.5 to 13.0 $\mathrm{ms} / \mathrm{mm}$ and during premature activation from 11.4 to $31.2 \mathrm{~ms} / \mathrm{mm}$. Likewise the relative inhomogeneity index measured during basic rinythm increased from 2.3 to 3.4 and during the earliest premature activation from 3.1 to $4.7\left(^{* *}=p<0.01 ;{ }^{* * *}=p<0.001 ;\right.$ student (-test).

times are termed "phase differences" because they express the highest degree at which adjacent sites are activated out of phase. The phase differences are influenced by the average speed of conduction, a high conduction velocity will decrease the phase differences whereas slow conduction will increase them. However, it should be stressed that the method of phase mapping is not suited for an accurate determination of the actual local conduction 
velocities, because the pathway of the impulse is not taken into account.

The overall conduction inhomogeneity in the investigated preparation was expressed by the P5-95 of all local phase differences (absolute inhomogeneity index). Because the population of phase differences was asymmetrically distributed, the distance between percentiles, instead of the standard deviation, was taken. An increase in the absolute inhomogeneity index is not necessarily caused by an increase in local conduction disturbances. It can also be due to a general depression in conduction velocity. An example of this is shown in figure 7.3 where, upon a decrease of the coupling interval of a premature activation from 100 to $70 \mathrm{~ms}$, the absolute inhomogeneity index increased from 6.3 to $7.6 \mathrm{~ms} / \mathrm{mm}$ while the median phase difference increased from 2.9 to $3.4 \mathrm{~ms} / \mathrm{mm}$. A distinction between a general decrease in conduction velocity and a true increase in conduction inhomogeneity can be made by calculating the relative inhomogeneity index (P5-P95/P50). In this case the relative inhomogeneity index remained constant at 2.2 implicating that the enhanced absolute inhomogeneity index was caused by a general increase in all phase differences. A further shortening of the coupling interval to $50 \mathrm{~ms}$ also increased the relative inhomogeneity index to 4.5 , indicating a "true" increase in inhomogeneity.

As shown in figure 7.2, conduction during slow regular rhythm is not completely homogeneous. Such inhomogeneities in conduction may be caused by the structural properties of the myocardium together with the direction of propagation of the impulse (Spach et al. 1981, 1982). The effect of the pacing site on the measured inhomogeneity can be explained by the different direction of the activation wave relative to the geometry of the preparation. If the orientation of an area of depressed conduction is perpendicular to the direction of a propagating impulse, it may cause a strong distortion of the activation wave. In case of an oblique orientation relative to the propagating wavefront only moderate inhomogeneity in conduction may result. When the impulse travels parallel to a line of conduction block it is even possible that no conduction disturbances are detected at all. This implicates that, for a complete quantification of inhomogeneity in conduction during sllow rhythm, pacing from different directions is necessary.

Inhomogeneity in conduction is enhanced during early premature beats. As shown by several authors (Alessi et al. 1958, Han et al. 1964 , 1966a) recovery in excitability is an inhomogenous process, some areas recovering more quickly than others. An early premature impulse will therefore conduct quickly in well recovered areas but 
propagate slowly or even block in areas where excitability is still low.

Induction of a series of multiple premature beats further increased inhomogeneity in conduction. The significant increase in inhomogeneity of the second premature beat (figure 7.5) indicates that the dispersion in recovery following the first premature beat has increased compared to the dispersion after the basic activation. This is in accordance with the increase in dispersion of recovery following a premature beat described by $\mathrm{Han}$ et al. (1964). Application of a third premature beat no longer significantly increased inhomogeneity in conduction, suggesting that dispersion in recovery after the second premature beat is not further enhanced. This however does not imply that maximal conduction disturbances are achieved by two successive premature beats. Actually a higher inhomogeneity in conduction was found during rapid pacing (table 7.1). Apparently, although after a sudden increase in pacing frequency inhomogeneity increases most markedly during the first few beats, inhomogeneity continues to develop more gradually during fast pacing until a stable value is reached. Janse et al. (1969) have studied the changes in refractory period following a sudden increase in pacing frequency and have described a sudden reduction in refractory period during the first few beats followed by a gradual decrease during subsequent beats, taking 100 to 500 beats before a new steady state was reached. 


\section{Chapter VIII}

\section{GENERAL DISCUSSION}

In the preceding chapters, the mechanisms underlying the initiation of intra-atrial reentry in two different arrhythmogenic situations have been investigated. In low-potassium a large reduction in refractoriness, together with a slight depression of the conduction velocity, induced a marked reduction in the wavelength of the impulse while inhomogeneity in conduction was not substantially altered. In contrast, hypoxia did not markedly shorten the wavelength but inhomogeneity in conduction was strongly enhanced. In this chapter, the relation between wavelength of the impulse and inhomogeneity in conduction will be discussed.

\subsection{Minimal length of conduction block for the initiation of reentry.}

Whether a conduction block is sufficiently long to induce reentry depends on several factors. To clarify the role of the wavelength and of inhomogeneity in conduction in reentry, a few diagrams are presented in figure 8.1. A premature impulse is initiated at the location of the stimulus symbol and isochrones are drawn in the usual way to visualize the spread of activation. The location of a temporary arc of conduction block is represented by the black bar. The phase differences on this line of conduction block, caused by the opposite direction of propagation of the impulse along both sides of the block, are plotted in the bar.

As shown in the first diagram (panel A), the occurrence of a line of conduction block diverts the impulse. First the impulse propagates from the site of stimulation upwards and downwards along the arc of conduction block. At the edges of the arc, the impulse changes its direction of propagation in a clockwise and a counterclockwise direction. Finally, both wavefronts propagate towards each other in the right part of the diagram to collide halfway the arc of conduction block. Because of the opposite direction of propagation along the two sides of the line of conduction block, the phase differences along the line of block gradually increase, starting with low values at the pivoting points to reach a maximum 

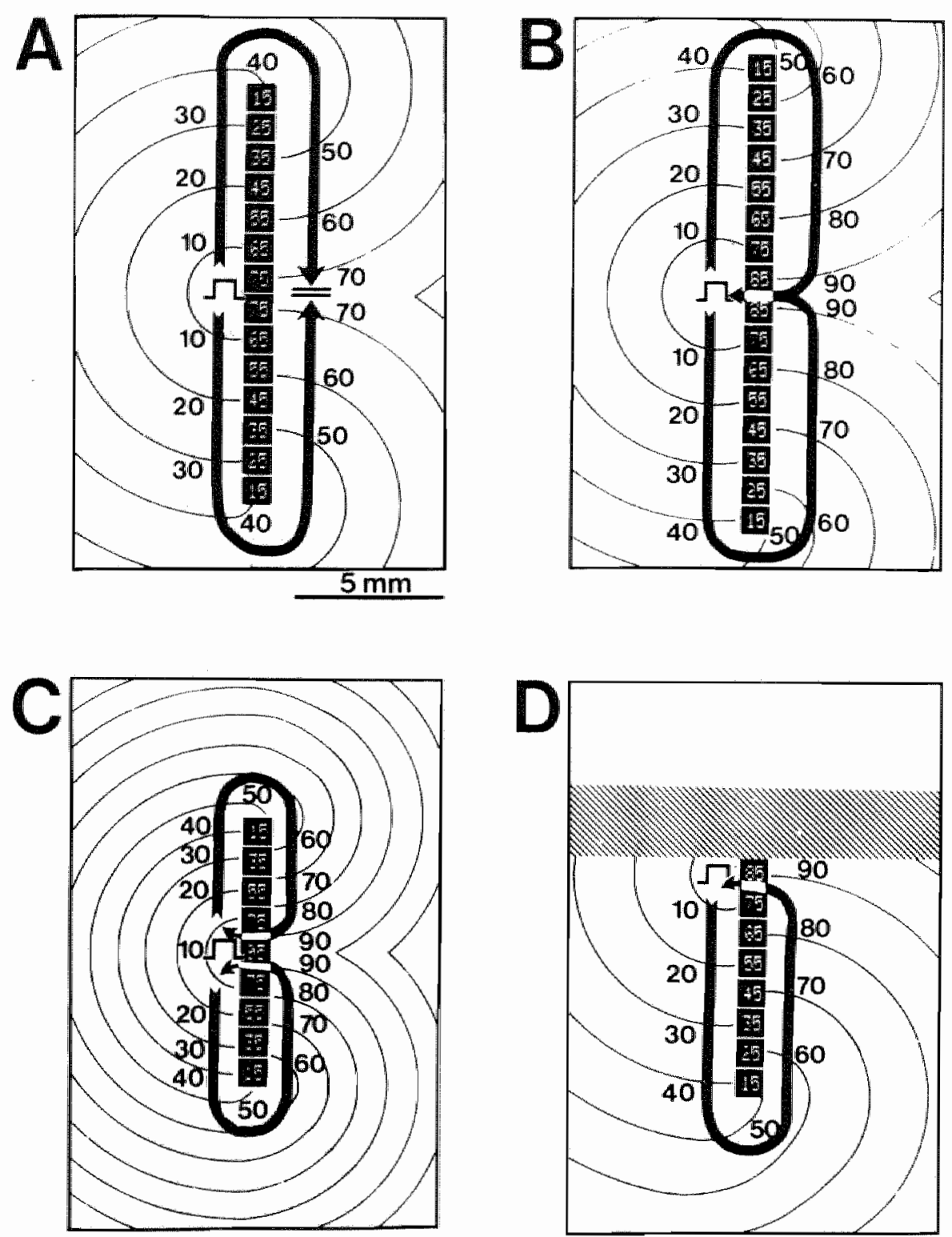

Figure 8.1. Size of a conduction block in relation to reentry. In each panel the location and size of the block is indicated by a black bar. Phase differences are plotted along the the line of conduction block as calculated from the difference in activation times at both sides of the line of block. A premature impulse is initiated at the location of the stimulus symbol. See text for further description. 
value halfway the arc. Both the length of the arc of block and the conduction velocity of the impulse propagating around the arc determine the moments of activation of the tissue at both sides of the block. An increase in length of the arc of conduction block (panel B) or a reduction in conduction velocity (panel C) will lead to dellayed activation, inducing a higher maximum phase difference halfway the arc of conduction block.

In order for reentry to occur, the area proximal to the block has to restore its excitability while the impulse is still propagating distal to the block. If we assume in figure 8.1 a refractory period of $80 \mathrm{~ms}$, the time elapsed during the roundabout conduction in panel $A$ is not sufficient to allow reentry. Along the line of block, the phase differences increase from 15 to $75 \mathrm{~ms}$. By the time the last part of the distal area is activated, the proximal area has been refractory for $75 \mathrm{~ms}$ and still requires $5 \mathrm{~ms}$ before it is excitable. In panel $B$ however, due to the increase in size of the arc of conduction block, the phase differences halfway the arc have become larger than the refractory period, the proximal area now having sufficient time for recovery of excitability.

Succesful reexcitation therefore is determined by the combination of refractory period, conduction velocity and length of the arc of conduction block. The refractory period and the conduction velocity are expressed together by the wavelength of the impulse (WL $=R P$ $x$ CV). Shortening of the wavelength, due to a reduction in the refractory period or a slowing in conduction, makes reentry more likely while lengthening of the wavelength makes it more difficult. The minimal length of the arc of conduction block necessary to enable reentry is determined by the size of the wavelength. In the propagation sequence presented in figure 8.1 (panels $A$ and B), a conduction velocity of $20 \mathrm{~cm} / \mathrm{sec}$ is assumed. The wavelength in panel A will than be $16 \mathrm{~mm}(80 \mathrm{~ms} \times 20 \mathrm{~cm} / \mathrm{sec})$. The length of the arc of block in panel $A$ is $14 \mathrm{~mm}$, which is not long enough to allow reexcitation. In panel $B$ the length of the arc of conduction block increased to $16 \mathrm{~mm}$, which is sufficient to permit reentry. In panel C, conduction velocity was depressed to $10 \mathrm{~cm} / \mathrm{sec}$, thereby reducing the wavelength to $8 \mathrm{~mm}(80 \mathrm{~ms} \times 10 \mathrm{~cm} / \mathrm{sec})$. Correspondingly, a length of the arc of block of $9 \mathrm{~mm}$ now is sufficiently long to allow reentry. In conclusion, when the site of the origin of a premature beat is adjacent to an area of conduction block, the length of the arc of block should be at least as long as the wavelength of the premature beat for reentry to occur.

Some modification of this rule however may occur depending upon the geometry of the myocardium. A closer examination of the 
excitation sequence in figure 8.1 panels A-C reveals that two loops are involved, each propagating along half the line of block. Upon sufficient lengthening of the arc of block, a common reentrant pathway is formed halfway the line of block and a figure of eight type of reentrant propagation is induced. Such a figure of eight can be seen occasionally during the induction of tachy-arrhythmias as shown in chapter 4 , figure 4.2. But in most cases, the situation was more like the one shown in figure 8.1 panel $D$ where a line of block is induced close to the border of the preparation. In our experiments, this was often the case due to the small size of the tissue. As shown in panel $D$, a single loop is formed around a single arc of conduction block. Again, the phase differences calculated along the line of block reached a sufficient magnitude to allow reexcitation of the proximal area. However, the length of the arc of conduction block now is only half the one shown in panel $B$, i.e. $8 \mathrm{~mm}$. In certain circumstances therefore, in the presence of an anatomic boundary, a minimal size of the arc of conduction black of only half the wavelength may be sufficient to induce reentry.

\subsection{Size of the central arc of conduction block during sustained reentry.}

Phase mapping makes it possible to locate and determine the length of arcs of conduction block in the center of sustained functional circus movement. In figure 8.2 panel $A$, a functional circus movement revolves around an arc of conduction block, delineated by a column of phase differences. At the edges of the arc, the phase differences are small due to the small difference in activation time before and after the impulse has pivoted around the line of block. Halfway the line of block, the phase difference has increased and is equal to half the cycle length of the circuit. With a phase difference of $55 \mathrm{~ms}$ (panel A), the cycle length will be 110 ms. The size of the arc of conduction block can be determined by the total length of the inter connected phase differences. For the rabbit left atrium, a minimal value of $14 \mathrm{~ms}$ phase difference, implicating a conduction velocity of $10 \mathrm{~cm} / \mathrm{sec}$ measured at an inter-electrode distance of $1.4 \mathrm{~mm}$, was chosen to indicate conduction block. In panel $A$, the size of the arc of block is $9 \mathrm{~mm}$. In figure 8.2 panel $B$, the phase differences in the center of a functional circus movement are reproduced from fig. 7.9. The largest phase difference is $41 \mathrm{~ms}$, which fits well with half the cycle length of the circuit $(80 \mathrm{~ms})$. The length of the arc of 

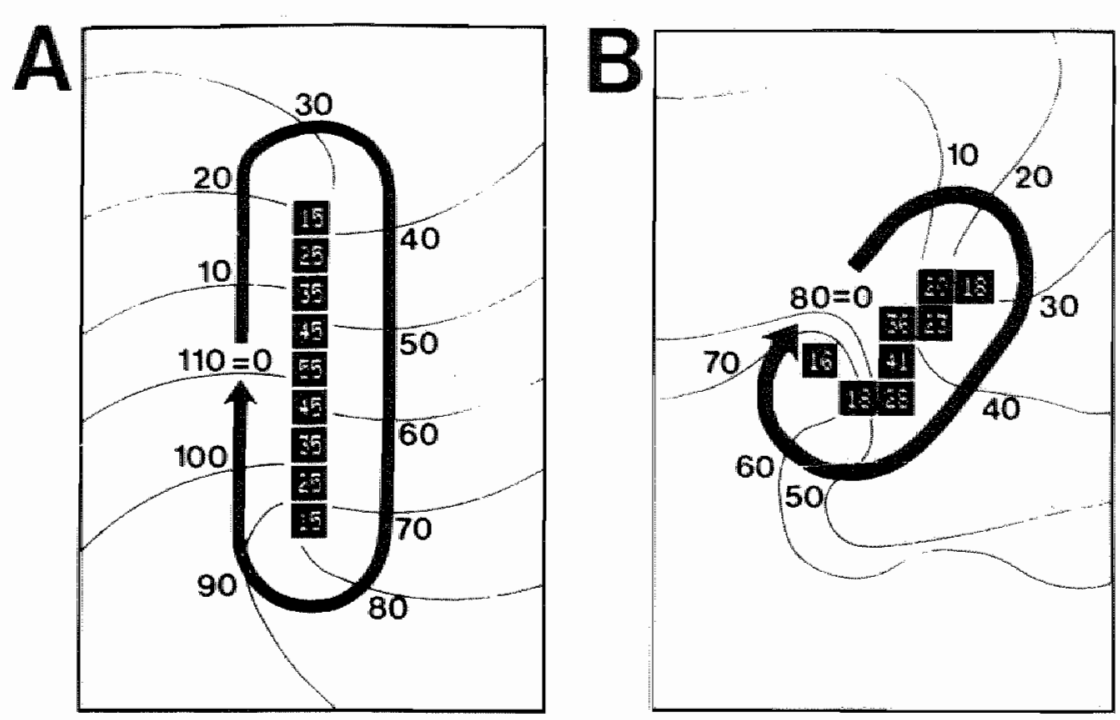

Figure 8.2. In panel $A$, a central arc of conduction block during a functional circus movement is shown by the black bar. Phase differences along the central arc of conduction block ranges from 15 to $55 \mathrm{~ms}$. In panel $B$, a similar central arc of conduction block is shown from a circulating excitation induced in a rabbit left atrial preparation. (chapter 7, figure 7.9). See tent for description.

conduction block is estimated at $11 \mathrm{~mm}$.

83 Size of conduction blocks and type of arrhythmia.

In a recent study, the critical lengths of the excitation wave to induce atrial fibrillation, atrial flutter or rapid repetitive responses have been measured in the dog (Rensma 1987). From these values, the length of conduction block necessary to induce these arrhythmias can be estimated. When the wavelength was longer than 12.3 $\mathrm{cm}$, arrhythmias were rarely induced. The minimal size of the arc of conduction block, at such a wavelength, must range between 6.2 and $12.3 \mathrm{~cm}$. Such elongated arcs of conduction block most probably do not often occur in the canine atria. At a critical wavelength of $9.7 \mathrm{~cm}$, atrial flutter was often induced, requiring a length of the arc of conduction block between 4.9 and $9.7 \mathrm{~cm}$. Below a wave- 
length of $7.8 \mathrm{~cm}$, atrial fibrillation frequently occurred. At these short wavelengths, an initial length of the arcs of conduction block of only 3.9 to $7.8 \mathrm{~cm}$ is sufficient to induce fibrillation. Obviously, such short arcs of conduction blocks readily occur in the normal canine atria during early premature activation or rapid stimulation.

The length of the arc of conduction block in the center of a functional circus movement also plays a role in the stability of the arrhyhthmia. As was shown in the activation maps of intra-atrial reentry induced in low-potassium, the central arc of conduction block is not fixed during the arrhythmia but may easily wander during the arrhythmia (chapter 4, figure 4.8). When such an arc of block reaches the border of the preparation, the impulse is blocked and the circus movement is terminated. Due to the small size of our preparation, this was often the case. In the situation of rapid repetitive activity in the canine atria, where the central arc of block is $10-12 \mathrm{~cm}$ long, the likelihood of a shift of the arc of block against a boundary such as the AV fibrous ring also seems high. This may explain the spontaneous termination of the reentrant process after a couple of beats.

At shorter wavelength however, the likelihood of a shift of the central arc of block against a boundary is smaller. Furthermore, in atrial fibrillation, multiple wavelets are simultaneously turning around several short arcs of conduction block (Allessie et al. 1985). In this situation, the chance that all wavelets are extinguished simultaneously is rather small, which may explain the higher stability of atriall fibrillation.

\subsection{Clinical significance of inhomogeneity in conduction.}

As demonstrated in the previous paragraphs, the length of arcs of conduction block necessary to induce reentrant arrhythmias can be estimated from the length of the excitation wave. Neither the size of arcs of conduction blocks, nor the length of the excitation wave, have as yet been measured in humans, except for a recent study in which a first estimation of the wavelength in the WPWsyndrome was made (Dugernier et al. 1986). Several studies have however measured refractory periods and conduction velocities in the human atria. During regular rhythm, the atrial refractory period is 220-260 ms (Attuel et al. 1982, Michelucci et al. 1985) and is shortened to about $150 \mathrm{~ms}$ upon premature stimulation. Premature conduction velocity is estimated at $80 \mathrm{~cm} / \mathrm{sec}$. The wavelength of an early premature beat will therefore be $12 \mathrm{~cm}$, and the length of 
the arc of conduction block, necessary to initiate circus movement, 6-12 cm. In the healthy human atria, such a large size of an arc of conduction block will rarely occur.

Several conditions however may change this situation, leading to an easier inducibility and an enhanced stability of arrhythmias. A pathological increase in the size of the atria is well known to be an important factor in the occurrence of atrial tachy-arrhythmias. Shortening of atrial refractoriness, thereby decreasing the wavelength of the impulse and reducing the length of the arc of conduction block necessary to induce reentry, is correlated with a higher inducibility for atrial fibrillation (Michelucci et al. 1982, Attuel et al. 1982, Olsson et al. 1982). Increase in inhomogeneity in conduction has also been reported (Cosio et al. 1983). Depolarized areas and slow action potentials, leading to local areas of slow conduction, have been found in tissues sampled from patients suffering from atrial arrhythmias (Hordof et al. 1976, Ten Eick et al. 1979, Mary-Rabine et al. 1983); furthermore, extensive degenerative changes have been shown in the atria of such patients (Davies et al. 1972, Salmon et al. 1985).

Whether a heart is susceptible for reentrant arrbythmias is determined by the balance between three factors: a) the available mass of tissue, b) the size of arcs of conduction block and c) the length of the excitation wave. Upon increase of the wavelength, both a larger tissue mass and longer arcs of conduction block are required for the initiation of reentry. Upon shortening of the wavelength, both the tissue mass and the length of conduction blocks can be smaller. Whether a reentrant arrhythmia, once started, will be sustained or not is also determined by the same three factors.

It is a challenge for the future to measure these three important parameters in the human heart. The methods for measurement of the wavelength (Rensma et al. 1987) and the quantification of inhomogeneity in conduction as presented in this thesis may provide the necessary tools to better understand the pathophysiological background for cardiac arrhythmias in man. 



\section{REFERENCES}

Akhtar M, Damato AN, Batsford WP, Ruskin JN, Ogunkelu JB, Vargas G: Demonstration of re-entry within the His-Purkinje system in man. Circulation, 50:1150-1162,1974.

Allessi $R$, Nusynowitz $M$, Abildskov JA, Moe GK: Nonuniform distribution of vagal effects on the atrial refractory period. Am J Physiol 194:406-410,1958.

Allessie MA, Bonke FIM, Schopman FJG: Circus movement in rabbit atrial muscle as a mechanism of tachycardia. Circ Res $32: 54-62,1973$.

Allessie MA, Bonke FIM, Schopman FJG: Circus movement in rabbit atrial muscle as a mechanism of tachycardia. II. The role of nonuniform recovery of excitability in the occurrence of unidirectional block,as studied with multiple microelectrodes. Circ Res 39:168-177,1976.

Allessie MA, Bonke FIM, Schopman FJG: Circus movement in rabbit atrial muscle as a mechanism of tachycardia. III. The "leading circle" concept: A new model of circus movement in cardiac tissue without the involvement of an anatomic obstacle. Circ Res 41:9-18,1977.

Allessie MA, Bonke FIM: Atrial Arrhythmias: Basic Concepts. In: Cardiac Arrhythmias. Their Mechanisms, Diagnosis and Management. Ed: Mandel WJ, J.B.Lippincott Company, Philaldelphia 1980.

Allessie MA, Lammers WJEP, Bonke FIM, Hollen J: Intra-atrial reentry as a mechanism for atrial flutter induced by acetylcholine and rapid pacing in the dog. Circulation 70:123-135,1984.

Allessie MA, Lammers WJEP, Bonke FIM, Hollen J: Experimental evaluation of Moe's multiple wavelet hypothesis of atrial fibrillation. In: Cardiac Electrophysiology and Arrhythmias. Ed: Zipes DP and Jalife J, Grune \& Stratton, Orlando 1985. 
Allessie MA, Schalij MJ, Wit AL, Lammers WJEP, Augustijn $\mathrm{CH}$ : Does anisotropy play a role in the creation of an excitable gap in reentrant ventricular tachycardia? In: Non-Pharmacological therapy of tachyarrythmias. Ed: Breithardt $G$, Futura Publishing Company, New York. 1987.

Anderson GJ, Greenspan K, Bandura JP, Fisch C: Asynchrony of conduction within the canine specialized Purkinje fiber system.

Circ Res 27:691-703, 1970 .

Antoni $H$, Zerweck $T$ : Besitzen die sympatischen Ubertragerstoffe einen direkten Einflusz auf die Leitungsgeschwindighkeit des Saugetiermyokards? Elektrophysiologische Untersuchungen an isolierten Papillarmuskeln und Purkinjefaden des Rhesusaffen. Pflügers Arch 293:310-330,1967.

Armitage AK, Burn JH, Gunning AJ: Ventricular fibrillation and ion transport. Circ Res 5:98-104,1957.

Attuel P, Childers R, Cauchemez B, Poveda J, Mugica J, Coumel P: Failure in the rate adaptation of the atrial refractory period: its relationship to vulnerability. Int J Cardiol 2:179-197,1982.

Barr RC, Spach MS: Sampling rates required for digital recording of 2intracellular and extracellular cardiac potentials. Circ Res 55:40-48,1977.

Billroth T: Briefe, edition 9. Hanover, Hahnsche, 1922, p 395. Cited by Shapiro E: Engelmann and his laddergram. Am $J$ Card 39:464-465,1977.

Boineau JP, Schuessler RB, Mooney CR, Miller CB, Wylds AC, Hudson RD, Borremans JM, Brockus CW: Natural and evoked atrial flutter due to circus movement in dogs. Am $J$ Cardiol 45:1167-1181,1980.

Boyden PA, Hoffman BF: The effects on atrial electrophysiology and structure of surgically induced right atrial enlargement in dogs. Circ Res 49:1319-1331,1981.

Brendel W, Raule W, Trautwein W: Die Leitungsgeschwindigkeit und Erregungsausbreitung in den Vorhofen des Hundes. Pflügers Arch 253:106-113,1950. 
Burdon-Sanderson J, Page FJM: On the time-relations of the 2excitatory process in the ventricle of the heart of the frog. J Physiol 2:384-435,1879.

Burdon-Sanderson J, Page FJM: On the electrical phenomena of the excitatory process in the heart of the frog and of the tortoise, as investigated photographically. J Physiol 4:327-338,1883.

Burgess MJ, Coyle J: Effects of premature depolarization on refractoriness of ischemic canine myocardium. J Electrocardiology 15:335-344,1982.

Buxton AE, Waxman HL, Marchlinski FE, Josephson ME: Atrial conduction: effects of extrastimuli with and without atrial dysrhythmias. Am J Cardiol 54:755-761,1984.

Cattermole KW: Principles of Pulse Code Modulation. lliffe Books, London, 1969.

Childers RW, Merideth J, Moe GK: Supernormality in Bachmann's bundle. An in vitro and in vivo study in the dog. Circ Res 22:363-370,1968.

Christe G: Effects of low $\mathrm{K}+$ on the electrical activity of human cardiac ventricular and Purkinje cells. Cardiovasc Res $17: 243-250,1982$.

Clerc L: Directional differences of impulse spread in trabecular muscle from mammalian heart. $J$ Physiol (London) 255:335-346,1976.

Cosio FG, Palacios J, Vidal JM, Cocina EG, Gomez-Sanchez MA, Tamargo L: Electrophysiologic studies in atrial fibrillation. Slow conduction of premature impulses: a possible manifestation of the background for reentry. Am J Card 51:122-130,1983.

Cranefield PF, Klein HO, Hoffman BF: Conduction of the cardiac impulse. I. Delay, block, and one-way block in depressed Purkinje fibers. Circ Res 28:199-219,1971a.

Cranefield PF, Hoffman BF: Conduction of the cardiac impulse. II. Summation and inhibition. Circ Res 28:220-233,1971b. 
Cranefield PF: The conduction of the Cardiac Impulse. The Slow Response and Cardiac Arrhythmias. Futura, Mount Kisko, New York, 1975.

Curry P, Fitchett D, Stubbs W, Krikler D: Ventricular arrhythmias and hypokalemia. Lancet 2:231-233,1976.

Dangman $\mathrm{KH}$, Danilo $\mathrm{P}$, Hordof AJ, Mary-Rabine $\mathbb{L}$, Reder RF, Rosen MR: Electrophysiological characteristics of human ventricular and Purkinje fibers. Circulation 65:362-368,1982.

Davies $M$, Pomerance A: Pathology of atrial fibrillation in man. $\mathrm{Br}$ Heart J 34:520-525,1972.

Dawes GS: Experimental cardiac arrhythmias and quinidine-like drugs. Pharmacol Rev 4:43-84,1952.

De la Fuente D, Sasyniuk BI, Moe GK: Conduction through a narrow isthmus in isolated canine atrial tissue. A model of the WPW syndrome. Circulation 44:803-809,1971.

Dillon S, Ursell PC, Wit AL: Pseudo-block caused by anisotropic conduction: a new mechanism for sustained reentry. Circulation 72:III-279,1985.

Dominguez G, Fozzard HA: Influence of extracellular $\mathrm{K}+$-concentration on cable properties and excitability of sheep cardiac Purkinje fibers. Circ Res 26:565-574,1970.

Downar E, Waxman MB: Depressed conduction and unidirectional block in Purkinje fibers. In: The Conduction System of the Heart. Ed: Wellens HJJ, Lie KI, Janse MJ. Stenfert Kroese, Leiden, 1976.

Downar E, Janse MJ, Durrer D: The effect of acute coronary occlusion on subepicardial transmembrane potentials in the intact porcine heart. Circulation 56:217-224,1977.

Draper MH, Mya-Tu M: A comparison of the conduction velocity in cardiac tissues of various mammals. Quart J Exptl Physiol 44:91-109, 1959 . 
Dugernier $T$, Brugada $P$, Lemery $R$, Cheriex $E$, Wellens HJJ: Application of the wave length concept to circus movement tachycardia (abstract). Circulation 74:II-259,1986.

Drury AN: Further observations upon intra-auricular block produced by pressure or cooling. Heart 12:143-169,1925.

Durrer D, Roos JP: Epicardial excitation of the ventricles in a patient with Woff-Parkinson-White syndrome (type B). Curculation 35:15-21,1967a.

Durrer D, Schoo L, Schuilenburg RM, Wellens HJJ: The role of premature beats in the initiation and the termination of supraventricular tachycardia in the Wolff-Parkinson-White syndrome. Circulation 36:644-662,1967b.

Durrer D, Wellens HJJ: The Wolff-Parkinson-White syndrome: anno 1973. Eur J Cardiol 1/4:347-367,1974.

Einthoven W: Un nouveau galvanometre. Arch $\mathrm{N}$ Sc Ex Nat $6: 625-633,1901$.

Eisner DA, Lederer WJ: Inotropic and arrhythmogenic effects of potassiumdepleted solutions on mammalian muscle. $J$ Physiol 294:255-277,1979.

El-Sherif N, Hope RR, Scherlag BJ, Lazzara R: Re-entrant ventricular arrhythmias in the late myocardial infarction period. II. Patterns of initiation and termination of reentry. Circulation 55:702-719,1977.

Engelmann ThW: Over de geleiding der irritatie in de spierzelfstandigheid van het hart. Koninkl Akad $v$ Wetensch Amsterdam. Proces Verbaal 15:12,1874.

Engelmann ThW: Ueber die leitung der Erregung im Herzmuskel. Pflüg Arch ges Physiol 11:465-480,1875.

Engelmann ThW: Over de geleiding der prikkels door het hart. Versl Kon Ak $v$ Wetensch. Zitting 25:93-96,1893. 
Engelmann ThW: Ueber reciproke und irreciproke Reizleitung mit besonderer Beziehung auf das Herz. Pfluegers Arch 61:275-284,1895.

Fournier M: Willem Einthoven - The Electrophysiology of the Heart. Medicamundi 21:65-70,1976.

Frame LH, Page RL, Hoffman BF: Atrial reentry around an anatomic barrier with a partially refractory excitable gap. Circ Res 58:495-511,1986.

Galvani L: De viribus electricitas in motu musculari commentarius. 1791

Garrey WE: The nature of fibrillary contraction of the heart. Its relation to tissue mass and form. Am J Physiol 33:397-414,1914.

Garrey WE: Auricular fibrillation. Physiol.Rev. 4:215-250,1924.

Gaskell WH: On the innervation of the heart, with especial reference to the heart of the tortoise. J. Physiol. 4:43-127,1883.

Gotch F: The succession of events in the contracting ventricle as shown by electrometer records - (tortoise and rabbit). Heart, 1:235-261,1910.

Grumbach L, Howard J, Merill J: Factors related to the initiation of ventricular fibrillation in the isolated heart: Effect of calcium and potassium. Circ Res 2:452-459,1954.

Han J, Moe GK: Nonuniform recovery of excitability in ventricular muscle. Circ Res 14:44-60,1964.

Han J, Millet D, Chizzonitti B, Moe GK: Temporal dispersion of recovery of excitability in atrium and ventricle as a function of heart rate. Am Heart $J$ 1:481-487,1966a.

Han J, de Jalon G, Moe GK: Fibrillation threshold of premature ventricular responses. Circ Res 18:18-25,1966b.

Han J, Moe GK: Cumulative effects of cycle length on refractory periods of cardiac tissues. Am J Physiol 217:106-109,1969. 
Harris AS, Matlock WP: The effects of anoxemic anoxia on excitability, conduction and refractoriness of mammalian cardiac muscle. Am J Physiol 150:493-503,1947.

Harvey W: An anatomical disputation concerning The Movement of the Heart and Blood in living creatures, 1578. Translated by $G$. Whitteridge. Blackwell Scientific Publications, 1976.

Hayden WG, Hurley EJ, Rytand DA: The mechanism of canine atrial flutter. Circ Res 10:496-505,1967.

Hiraoka M, Okamoto $\mathrm{Y}$, Sano $\mathrm{T}$ : Effects of $\mathrm{Ca}+$ and $\mathrm{K}+$ on oscillatory afterpotentials in $\operatorname{dog}$ ventricular muscle fibers. $J$ Mol Cell Card 11:999-1015,1979.

Hiraoka M, Okamoto $Y$, Sano T: Oscillatory afterpotentials in $\operatorname{dog}$ ventricular fibers. Circ Res 48:510-518,1981.

Hoff HE, Geddes LA: The rheotome. Bull Hist Med 31:212-234 and $327-347,1957$.

Hoffa M, Ludwig C: Einige neue Versuche uber Herzbewegung. Zeitschrift fur Rat Med 9:107-144,1850.

Hoffman BF, Cranefield PF: Electrophysiology of the heart. McGraw-Hill, New York, 1960.

Hoffman BF, Rosen MR: Cellular mechanisms for cardiac arrhythmias. Circ Res 49:1-15,1981.

Holland WC, Burn JH: Production of fibrillation in isolated atria of rabbit heart. Br Med J 1:1031-1033,1954.

Holland WC, Tinsley B: Factors affecting the incidence of atrial fibrillation. Amer J Physiol 193:235-238,1958a.

Holland WC, Klein RL: Effects of temperature, $\mathrm{Na}$ and $\mathrm{K}$ concentration and Quinidine on transmembrane flux of $\mathrm{K} 42$ and incidence of atrial fibrillation. Circ Res 6:516-521,1958b.

Hondeghem LM, Grant AO, Jensen RA: Antiarrhythmic drug action: selective dpression of hypoxic cardiac cells. Am Heart J 87:602-605,1974. 
Hordof Al, Edie $R$, Malm JR, Hoffman $B F$, Rosen MR: Electrophysiologic properties and response to pharmacologic agents of fibers from diseased human atria. Circulation $54: 774-779,1976$.

linuma $H$, Kazuzo $\mathrm{K}$ : The effect of hypoxia on the refractoriness of the canine ventricular muscle. I Electrocard 11:15-22,1978.

Jacobson ED, Schiess W, Moe GK: The effect of hypoxia on experimental ventricular tachycardia. Am Heart $J$ 64:368375,1962

Janse MJ, Van der Steen BM, Van Dam RT, Durrer D: Refractory period of the dog ventricular myocardium following sudden changes in frequency. Circ Res 24:251-262,1969

Janse MJ: The effects of changes in heart rate on the refractory period of the heart. Thesis, University of Amsterdam, 1971.

Janse MJ, Downar E: The effect of acute ischemia on transmembrane potentials in the intact heart. The relation to re-entrant arrhythmias. In: Reentrant Arrhythmias, Ed: Kulbertus HE, MTP Press, Lancaster, 1977.

Janse MJ: Reentry rhythms. In: The Heart and Cardiovascular System. Scientific Foundations. Ed: HA Fozzard, E Haber, RB Jennings, AM Katz, HE Morgan. Raven Press, New York, 1986.

Joyner RW, Overholt ED, Ramza B, Veenstra RD: Propagation through electrically coupled cells: Two inhomogeneously coupled cardiac tissue layers. Am J Physiol 247:H596-H609,1984.

Kent AFS: Observations on the auriculo-ventricular junction of the mammalian heart. Q J Exp Physiol 7:193-195,1913.

Kimura E, Kato K, Murao S, Ajisaka H, Koyama S, Omiya Z: Experimental studies on the mechanism of the auricular flutter. Tohoku J Exp Med 60:197-20,1954. 
Kimura S, Bassett AL, Kohya T, Kozlovskis PL, Myerburg RJ: Simultaneous recordings of action potentials from endocardium and epicardium during ischemia in the isolated cat ventricle: relation of temporal electrophysiologic heterogeneities to arrhythmias. Circulation 74:401-409,1986.

Kuisseleff M: Der Ringrhythmus an Nerv-Muskelpreparaten. Pflügers Arch 226:99-13,1930.

Kolliker A, Muller H: Nachweis der negativen Swankung des Muskelstroms am naturlich sich contrahierenden Muskel.

Verhandl der Phys Med Gesellschaft in Wurzburg 6:529,1855.

Kronecker $H$, Schmey $F$ : Das Coordinationscentrum der Herzkammerbewegungen. Sitzungs Berichte Akad d Wiss zu Berlin $87-89,1884$.

Kunin AS, Surawicz B, Sims E: Decrease in serum potassium concentrations and appearance of cardiac arrhythmias during infusion of potassium with glucose in potassium-depleted patients. New Engl J Med 266:228-233,1962.

Kuo CS, Munakata K, Reddy P, Surawicz B: Characteristics and possible mechanism of ventricular arrhythmia dependent on the dispersion of action potential durations. Circulation 67:1356-1367,1983.

Lanari A, Lambertini A, Ravin A: Mechanism of experimental atrial flutter. Circ Res 4:282-287,1956.

Lammers WJEP, Wit AL, Allessie MA: Effects of anisotropy on functional reentrant circuits" Preliminary results of computer simulation studies. In: Simulation and modeling of the cardiac system: from cellular activation to muscular activity. Ed: Sideman S, Beyar R. 1987.

Lazzara R, Hope RR, El-Sherif $\mathrm{N}$, Scherlag BJ: Effects of lidocaine on hypoxic and ischemic cardiac cells. Am J Card $41: 872-879,1978$.

Legato MJ, Bull MB, Ferrer MI: Atrial ultrastructure in patients with fixed intra-atrial block. Chest 65:252-261,1974. 
Lewis T, Meakins $J_{*}$ White PD: The excitatory process in the dog's heart. Part I. The auricles. Philos Trans $R$ Soc London 205:375-420,1914.

Lewis $T$, Rothschild MA: The excitatory process in the dog's heart. Part II. The ventricles. Philos Trans $R$ Soc London 206:181-226,1915.

Lewis $T$, Feil HS, Stroud WD: Observations upon flutter and fibrillation. Part II. The nature of auricular flutter. Heart VII:191-246,1920.

Lewis T, Drury AN, Bulger HA: Observations upon flutter and fibrillation. Part VI. The refractory period and rate of propagation in the auricle: Their relation to block in the auricular walls and to flutter, etc. Heart 8,83-140,1921.

Lewis $\mathrm{T}$ : The mechanism and graphic registration of the heart beat. 3rd ed., Shaw and Sons, London 1925.

Lindeboom GA: De verdiensten van Jan Swammerdam voor de medische wetenschap. Ned.T.Geneesk. 124:2135-2141,1980.

MacWilliam JA: Fibrillar contraction of the heart. J Physiol 8:296-310,1887.

Mallinson JC, Miller JW: On Optimal Codes for Digital Magnetic Recording. Ampex Corporation Data Systems Division, Redwood City, California 94063. Ampex Bulletin D364, 1979.

Marey EJ: Des variations electriques des muscles et du coeur en particulier etudiees au moyen de l'electrometre de M Lippman. CR Acad Sci 82:975,1876.

Mary-Rabine L, Albert A, Pham TD, Hordof AL, Fenoglio $\mathrm{Jr}_{r} \mathrm{JJ}$, Malm JR, Rosen MR: The relationship of human atrial cellular electrophysiology to clinical function and ultrastructure. Circ Res 52:188-199,1983.

Mayer AG: Rhythmical pulsation in scyphomedusae. Carnegie Institution Pubication No. 47:1,1906. 
Mayer AG: Rhythmical pulsation in scyphomedusae.-II. Papers from the Tortugas Laboratory of the Carnegie Institution of Washington. Publication no. 102:115,1908.

Mehra R, Zeiler RH, Gough WB, El-Sherif N: Reentrant ventricular arrhythmias in the late myocardial infarction. 9. Electrophysiologic anatomic correlation of reentrant circuits. Circulation 67:11-24,1983.

Mendez C, Mueller WJ, Urguiaga X: Propagation of impulses across the Purkinje fiber-muscle junctions in the dog heart. Circ Res 26:135-150,1970.

Merx W, Yoon MS, Han JN: The role of disparity in conduction and recovery time on ventricular vulnerability to fibrillation. Am Heart J 94:603-610,1977.

Michelucci A, Padeletti L, Fradella GA: Atrial refractoriness and spontaneous or induced atrial fibrillation. Acta Cardiol 37:333-344,1982.

Michelucci A, Padeletti L, Fradella GA, Monizzi D, Giomi A, Fantini F: Effects of autonomic blockade on atrial electrophysiologic properties in normal subjects and in patients with sinus node disease. Int J Cardiol 8:437-445,1985.

Mines GR: On dynamic equilibrium in the heart. J Physiol 46:349-383,1913.

Mines GR: On circulating excitations in heart muscles and their possible relation to tachycardia and fibrillation. Trans, $R$ Soc of Canada, Section V:43-53,1914.

Moe G.K: On the multiple wavelet hypothesis of atrial fibrillation. Arch Int Pharmacodyn Ther 140:183-188,1962.

Moe GK, Rheinboldt WC, Abildskov JA: A computer model of atrial fibrillation. Am Heart J 67:200-220,1964.

Moe GK, Mendez C, Han J: Aberrant A-V impulse propagation in the dog heart: a study of functional bundle branch block. Circ Res 16,261-286,1965. 
Moe GK, Pastelin $G$, Mendez $R$ : Circus movement excitation of the atria. In: Physiology of atrial pacemakers and conductive tissue. Ed: Little RC, New York, Futura Publishing Co., 1980.

Mugelli A, Mantelli L, Manzini S, Ledda F: Induction by histamine of oscillatory activity in sheep Purkinje fibers and suppression by verapamil and lidocaine. J Cardiow Pharm 2:9-15,1980.

Myerburg RJ, Nilsson $\mathrm{K}$, Befeler B, Castellanos A, Gelband H: Transverse spread and longitudinal dissociation in the distal A-V conducting system. J Clin Invest 52:885-895,1973.

Naimi S, Avitall B, Mieszala J, Levine H: Dispersion of effective refractory period during abrupt reperfusion of ischemic myocardium in dogs. Am J Card 39:407-412,1977.

Neuss $H$, Schlepper $M$, Thormann J: Analysis of re-entry mechanism in three patients with concealed Wolff-Parkinson-White syndrome. Circulation 51:75-81,1975.

Nishi K, Yoshikawa Y, Takenaka F, Akaike $\mathrm{N}$ : Electrical activity of sinoatrial node cells of the rabbit surviving a long exposure to cold Tyrode's solution. Circ Res 41:242-247,1977.

Nordrehaug JE, Lippe $\mathrm{G}$ von der: Hypokalaemia and ventricular fibrillation in acute myocardial infarction. $\mathrm{Br}$ Heart $\mathrm{J}$ 50:525-529,1983.

Olsson SB, Brorson L, Varnauskas E: Monophasic action-potential studies of atrial fibrillation. In: Atrial fibrillation. Ed: Kulbertus HE, Olsson SB, Schlepper M, Sweden, AB Haessle, 1982.

Paes de Carvalho A, Saldena TA, Garcia EAC, Campos de Carvalho AC, Tashiro $T$, Paula de Carvelho $M$ : Homogeneous and nonhomogeneous conduction of impulses in heart and other excitable tissues. In: Normal and Abnormal Conduction in the Heart. Ed: Paes de Carvalho, Hoffman BF, Lieberman M. Futura Publishing Company, Mount Kisko, New York, 1982.

Page P, Plumb V, Waldo AL: Total epicardial mapping of atrial flutter in a new animal model (abstract). J Am Coll Cardiol $1: 716$ A, 1983 . 
Pastelin G, Mendez $R$, Moe GK: Participation of atrial specialized conduction pathways in atrial flutter. Circ Res 42:386-393,1978.

Plumb VJ, Karp RB, James TN, Waldo AL: Atrial excitability and conduction during rapid atrial pacing. Circulation $63,1140-1149,1981$.

Porter WT: The recovery of the heart from fibrillary contractions. Am J Physiol 1:71-82,1898. Porter WT: The coordination of the ventricles. Am J Physiol 2:127-136,1899.

Prasad K: Cardiac metabolism and electromechanics of human heart. In: Recent advances in studies on cardiac structure and metabolism 10:119-137,1975.

Ravens KG, Ravens $U$ : The role of linoleic acid in hypoxia-induced changes of the action potentials and the force of contraction of isolated papillary muscles of the guinea pig. In: Recent advances in studies on cardiac structure and metabolism. Ed: Harris P, Bing RJ, Fleckenstein A 7:289-295,1976.

Rensma PL: Atrial arrhythmias and measurement of the wavelength in a chronic dog model. Thesis. University of Limburg 1987.

Rensma PL, Allessie MA, Lammers WJEP, Bonke FIM, Schalij MJ: The length of the excitation wave and the susceptibility to reentrant atrial arrhythmias in normal conscious dogs. In Press, 1987.

Resnik WH: Observations on the effect of anoxemia on the heart. III. Changes in the auricles, with particular reference to the relationship between anoxemia and auricular fibrillation. $J$ Clinical Invest 2:125-141,1925.

Romanes GJ: Jelly fish, star fish and sea urchins. p 67, 1885.

Rosenblueth A, Garcia Ramos J: Studies on flutter and fibrillaton. II. The influence of artificial obstacles on experimental auricular flutter. Am Heart J 33:677-684,1947.

Saito $T$, Otoguro $M$, Matsubara $T$ : Electrophysiological studies on the mechanism of electrically induced sustained rhythmic activity in the rabbit right atrium. Circ Res 42:199-206,1978. 
Salmon DR, McPherson DD, Augustine DE, Holida MD, White CW: A canine model of chronic atrial fibrillation: echocardiographic and electrocardiographic validation (abstract). Circulation 72:III-250,1985.

Sano T, Takayama N, Shimamoto $T$ : Directional difference of conduction velocity in the cardiac ventricular syncytium studied by microelectrodes. Circ Res:262-267,1959.

Sarachek NS, Roberts $J$, Leonard JJ: A new method to measure nonuniformity in the intact heart. $J$ Electrocardiology 5:341-348,1972.

Saunders JH, Ferrier GR, Moe GK: Conduction block associated with transient depolarizations induced by acetylstrophanthidin in isolated canine Purkinje fibers. Circ Res 32,610-617,1973.

Schmitt $F O_{n}$ Erlanger $J:$ Directional differences in the conduction of the impulse through heart muscle and their possible relation to extrasystolic and fibrillary contractions. Am J Physiol 87:326-347,1928.

Senges $J$, Mizutani T, Pelzer $D$, Brachmann J, Sonnhof U, Kubler W: Effect of hypoxia on the sinoatrial node, atrium, and atrioventricular node in the rabbit heart. Circ Res 44:856-863,1979.

Shimizu T, Iwamura N, Toyama J, Yamada K: Inhomogeneous spread of excitation in canine Purkinje fibers. Jap Heart $J$ 20:511-522,1979.

Siegenbeek van Heukelom $\mathfrak{J}$, Dijkstra $K$, Ingen $H$ van: $A$ versatile and reproducibly operating microelectrode puller. Med Biol Eng 644-652,1976.

Simpson RJ, Foster JR, Gettes LS: Atrial excitability and conduction in patients with interatrial conduction defects. Am $\mathrm{J}$ Cardiol 50:1331-1337,1982.

Singer $\mathrm{DH}$, Lazzara $\mathrm{R}$, Hoffman BF: Interrelationships between automaticity and conduction in Purkinje fibers. Circ Res 21:537-558,1967. 
Smeets JLRM: Wavelength of the cardiac impulse and reentrant arrhythmias. Thesis. University of Limburg 1983.

Smeets JLRM, Allessie MA, Lammers WJEP, Bonke FIM, Hollen J: The wavelength of the cardiac impulse and reentrant arrhythmias in isolated rabbit atrium. Circ Res 58:96-108,1986.

Smith JR, Wilson KS: Studies on the production and maintenance of experimental auricular fibrillation. Am Heart J 176-185,1943

Smith WM, Ideker RE, Kinicki RE, Harrison L: A computer system for the intraoperative mapping of ventricular arrhythmias. Comp and Biomed Res 13:61-72,1980.

Snellen HA: History of Cardiology; a brief outline of the 350 years' prelude to an explosive growth. Donker, Rotterdam, 1984.

Spach MS, Miller WT, Miller-Jones E, Warren RB, Barr RC: Extracellular potentials related to intracellular action potentials during impulse conduction in anisotropic canine cardiac muscle. Circ Res 45:188-204,1979.

Spach MS, Miller WT Jr, Geselowitz DB, Barr RC, Kootsey JM, Johnson EA: The discontinuous nature of propagation in normal canine cardiac muscle. Evidence for recurrent discontinuities of intracellular resistance that affect the membrane currents. Circ Res 48:39-54,1981.

Spach MS, Miller WT, Dolber PC, Kootsey M, Sommer JR, Mosher $\mathrm{CE}$ : The functional role of structural complexities in the propagation of depolarization in the atrium of the dog. Cardiac conduction disturbances due to discontinuities of effective axial resistivity. Circ Res 50:175-191,1982.

Spach MS, Dolber PC: Relating extracellular potentials and their derivatives to anisotropic propagation at a microscopic level in human cardiac muscle. Circ Res 58:356-371,1986a.

Spach MS, Dolber PC, Kootsey JM, Johnson EA: Accounting for anisotropic behavior of propagation by directional differences of membrane capacity in human cardiac muscle. Circulation 74:II-419,1986b. 
Spear JF, Moore EN: Supernormal excitability and conduction in the His-Purkinje system of the dog. Circ Res 35:782-792,1974a.

Spear JF, Moore EN: The effets of changes in rate and rhythm on supernormal excitability in the isolated Purkinje system of the dog. A possible role in reentrant arrhythmias. Circulation 50:1144-1149,1974b.

Sperelakis $N$, Mayer $G$, MacDonald $R$ : Velocity of propagation in vertebrate cardiac muscles as functions of tonicity and $\mathrm{K}+$. Am J Physiol 219:952-963,1970.

Steinberg CA, Paine LW: Methods and Techniques of Data Conversion. Ann N Y Acad Sci 115,1964.

Steiness $\mathrm{E}$, Olesen $\mathrm{KH}$ : Cardiac arrhythmias induced by hypokalemia and potassium loss during maintenance digoxin therapy. $\mathrm{Br}$ Heart J 38:167-172,1976.

Surawicz B: The interrelationship of electrolyte abnormalities and arrhythmias. In: Cardiac arrhythmias. Their Mechanisms, Diagnosis and Management. Ed: Mandel WJ, Lippincott company, Philadelphia, 1980.

Tasaki K, Tsukahara $Y$, Ito $S$, Wayer MJ, Yu WA: A simple, direct and rapid method for filling microelectrodes. Physiol Behay 3:1009-1010,1968.

Teiger D, Scheider F, Farah A: The effects of sodium ions and rate of stimulation on the refractory period of isolated rabbit atrial muscle. J Pharmacol Exp Ther 155:58-68,1967.

Ten Eick RE, Singer DH: Electrophysiological properties of diseased human atrium. I. Slow diastolic potential and altered cellular response to potassium. Circ Res 44:545-557,1979.

Trautwein W, Gottstein U, Dudel J: Der aktionsstrom der myokardfaser im sauerstoffmangel. Pflügers Arch 260:40-60,1954.

Van Capelle FJL, Janse MJ: Influence of geometry on the shape of the propagated action potential. In: The Conduction System of

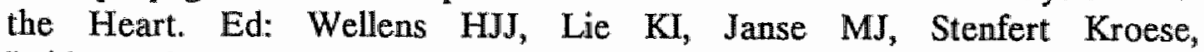
Leiden, 1976. 
Veenstra R, Joyner RW, Rawling D: Purkinje and ventricular activation sequences of canine papillary muscle: Effects of quinidine and calcium on Purkinje-ventricular conduction delay. Circ Res 54:500-515,1984.

Vleugels A, Carmeliet $E$, Bosteels $S$, Zaman $M$ : Differential effects of hypoxia with age on the chick embryonic heart. Changes in membrane potential, intracellular $\mathrm{K}$ and $\mathrm{Na}$, K-efflux and glycogen. Pflügers Arch 365:159-166,1976.

Waart de A: Het levenswerk van Willem Einthoven 1860-1927. Erve F. Bohn, Haarlem, 1957.

Wallace AG, Mignone RJ: Physiologic evidence concerning the reentry hypothesis for ectopic beats. Am Heart J 72:60-70,1966.

Webb JL, Hollander PB: Metabolic aspects of the relationship between the contractility and membrane potentials of the rat atrium. Circ Res 4:618-626,1956.

Weidmann S: Effects of calcium ions and local anesthetics on electrical properties of Purkinje fibres. J Physiol (London) 129:568-582,1955.

Wellens HJJ: Electrical stimulation of the heart in the study and treatment of tachycardias. Stenfert Kroese, Leiden, 1971.

Wellens HJJ, Schuilenburg RM, Durrer D: Electrical stimulation of the heart in patients with ventricular tachycardia. Circulation $46,216-226,1972$.

Wellens HJJ, Lie $\mathrm{KI}$, Durrer D: Further observations on ventricular tachycardia as studied by electrical stimulation of the heart. Chronic recurrent ventricular tachycardia and ventricular tachycardia during acute myocardial infarction. Circulation 49:647-653,1974.

Wellens HJJ: Pathophysiology of ventricular tachycardia in man. Arch Intern Med 135:473-479,1975a.

Wellens HJJ: Contribution of cardiac pacing to our understanding of the Wolff-Parkinson-White syndrome. $\mathrm{Br}$ Heart J 37:231-241,1975b. 
Wellens HJJ, Durrer D: The role of an accessory atrioventricular pathway in reciprocal tachycardia. Circulation 52:58-72,1975c.

Wellens HJJ, Farre J, Bar FW: The Wolff-Parkinson-White syndrome. In: Cardiac Arrhythmias. Their Mechanisms, Diagnosis and Management. Ed: Mandel. JB Lippincott, Philadelphia, 1980.

Wellens HJ, Brugada P: Value of programmed stimulation of the heart in patients with the Wolff-Parkinson-White syndrome. In: Tachycardias-Mechanisms, diagnosis and treatment. Ed: Josephson ME, Wellens HJJ. Lea and Febiger, Philadelphia, 1984.

Wennemark JR, Ruesta VJ, Brodly DA: Microelectrode study of delayed conduction in the canine right bundle branch. Circ Res 23:753-769,1968.

West TC, Cox AR: Single fiber recording during the production and control of flutter in the isolated atrium of the rabbit. J Pharmacol Exp Ther 130:303-310,1960.

White CW, Kerber RE, Weiss HR, Marcus ML: The effects of atrial fibrillation on atrial pressure-volume and flow relationships. Circ Res 51:205-215,1982.

Wiener N, Rosenblueth A: The mathematical formulation of the problem of conduction of impulses in a network of connected excitable elements, specifically in cardiac muscle. Arch Inst Cardiol Mex 16:205-265,1946.

Wit AL, Hoffman BF, Cranefield PF: Slow conduction and reentry in the ventricular conducting system. I. Return extrasystole in canine Purkinje fibers. Circ Res 30:1-10,1972a.

Wit AL, Hoffman BF, Cranefield PF: Slow conduction and reentry in the ventricular conducting system. II. Single and sustained circus movement in networks of canine and bovine Purkinje fibers. Circ Res 30:11-22,1972b.

Wit AL, Allessie MA, Bonke FIM, Lammers WJEP, Smeets JLRM, Fenoglio Jr JJ: Electrophysiologic mapping to determine the mechanism of experimental ventricular tachycardia initiated by premature impulses. Experimental approach and initial results demonstrating reentrant excitation. Am J Card 49:166-185,1982. 
Witkowski FX, Corr PB: An automated simultaneous transmural cardiac mapping system. Am J Physiol 247:H661-H668,1984.

Wojtczak $\mathrm{J}$ : Contractures and increase in internal longitudinal resistance of cow wentricular muscle induced by hypoxia. Circ Res 44:88-95,1979.

Wyndham CRC, Amat-y-Leon F, Wu D, Denes P, Dhingra R, Simpson $R$, Rosen $K$ : Effects of cycle length on atrial vulnerability. Circulation 55:260-267,1977.

Zipes DP, Mihalick MJ, Robbins GT: Effects of selective vagal and stellate ganglion stimulation on atrial refractoriness. Cardiovasc Res 8:647-655,1974. 



\section{SUMMARY}

The purpose of the study presented in this thesis was to evaluate the role of inhomogeneity in conduction in the initiation of reentrant tachy-arrhythmias. Normal and abnormal conduction of the cardiac impulse have been the subject of numerous investigations dating back to the previous century (chapter I). From these studies information concerning conduction and conduction disturbances were gathered but, due to technical limitations, a quantitative description of inhomogeneity in conduction has been lacking.

Chapter III describes the method used in this study. The preparation consisted of the isolated left atrium of the rabbit superfused with Tyrode. Conduction of the impulse during normal propagation and during the initiation of reentry could be analyzed in detail by recording the electrical activity simultaneously from 192 recording electrodes. Tachy-arrhythmias were initiated either in normal superfusing conditions or in a more arrhythmogenic situation, induced by lowering the potassium-or the oxygen-concentration.

The characteristics of the atrial tachy-arrhythmias induced in a low-potassium environment are presented in chapter IV. Mapping the spread of activation during these arrhythmias revealed that the majority of the arrhythmias were caused by functional circus movement whereas a few arrhythmias were based on abnormal impulse formation. Such an increase in reentrant tachy-arrhythmias may be due either to a shortening of the wavelength of the impulse or to an increase in inhomogeneity in conduction. We measured the length of the excitation wave during premature activation and showed that the wavelength was markedly shortened during lowpotassium superfusion. On the other hand, no evidence for an increase in inhomogeneity in conduction was obtained. The higher incidence of reentrant tachy-arrhythmias in low-potassium is therefore caused by the shortening of the length of the cardiac impulse.

The studies on the mechanism of arrhythmias induced in hypoxia are presented in chapter V. Again, detailed analysis of the activation sequence revealed that the majority of the arrhythmias were based on functional reentrant activity although a few arrhythmias, based on focal activity, were also found. In contrast to the studies 
performed in low-potassium, the wavelength during hypoxia was not markedly shortened. Propagation of the premature impulse however seemed to proceed more inhomogeneously in hypoxia.

Inhomogeneous conduction may be due either to structural or to functional properties of the myocardium. The structural properties are determined by the architecture of the cardiac fibers. Inhomogeneous distribution of the fibers will lead to an inhomogeneous pattern of propagation. Functional properties is for example present during the recovery of a previous excitation. In chapter VI, we found that recovery of excitability, as measured at different sites in the atrium, proceeds more asynchronously in hypoxia than during normal superfusion. Such an increased dispersion in recovery will in turn lead to a more inhomogenous propagation of a premature wavefront which encroaches on the inhomogeneously retreating tail of refractoriness.

In order to evaluate this phenomena, we quantified the number of conduction blocks occurring during premature activation both during control superfusion and during hypoxia. As shown in chapter VI, hypoxia induced a significant increase in the occurrence of conduction blocks during premature activation compared to control.

The occurrence of conduction block is only one sign of an increased inhomogeneity in conduction. In chapter VII, a more general method of visualizing and quantifying inhomogeneity in conduction is presented. The method consists of calculating the maximum difference in activation times recorded between neighboring recording sites ( $=$ phase difference). From these local phase differences a phase map can be constructed, describing the spatial distribution in local inhomogeneity in conduction. Furthermore, an index of inhomogeneity can be calculated by taking the spread in the population of phase differences.

In a normal superfused preparation, the effects of pacing at a slow rhythm, of single and multiple premature beats and of rapid pacing on the conduction properties were investigated. During slow rhythm, inhomogeneity in conduction was dependent on the site of stimulation and revealed the structural conduction properties of the preparations. The degree of inhomogeneity in conduction was increased during premature activation and during rapid pacing. Moreover, during rapid pacing, conduction inhomogeneities occurred throughout the preparation whereas during premature stimulation they were confined to the vicinity of the stimulating electrode.

The relation between reentrant arrhythmias and inhomogeneity in conduction was also investigated using the multiple recording system. Activation maps recorded during the initiation of a circus 
movement arrhythmia localized the site of reentry. The corresponding phase maps revealed that the site of reentry correlated with areas with highest local phase differences. Finally, application of the phase method during the perpetuation of the arrhythmias revealed that the degree of inhomogeneity in conduction was highest during the initiation of the arrhythmia. After its initiation, the inhomogeneity index dropped to a much lower level and gradually increased to a stable value during the time course of the arrhythmia.

In chapter VIII, a general discussion on the correlation between the wavelength of the cardiac impulse, inhomogeneity in conduction and reentrant arrhythmias is presented. In the absence of anatomical boundaries, the arc of conduction block should be at least as long as the wavelength for reentry to occur. In the presence of anatomical boundaries, the length of the arc of conduction block may be reduced to half the length of the excitation wave and still allow reentry to occur. Furthermore, the clinical significance of quantifying inhomogeneity in conduction is discussed.

We conclude that inhomogeneity in conduction, together with the wavelength of the cardiac impulse and the available tissue mass, is a major determinant for the occurrence of reentrant tachy-arrhythmias. It is furthermore of importance to quantify the effect of cardiac drugs on inhomogeneity in conduction. Finally, the method of phase mapping makes it possible to quantify the degree of inhomogeneity in conduction in patients afflicted with reentrant arrhythmias. 



\section{SAMENVATTING}

De normale harstlag wordt veroorzaakt door het min of meer gelijktijdig samentrekken van de miljoenen individuele cellen waaruit het hart is samengesteld. Het signaal voor elke hartcel om te contraheren wordt gegeven door een electrische impuls die zich over de oppervlakte van deze hartcellen voortplant. Onder normale omstandigheden worden de kamers van het hart binnen $0.1 \mathrm{sec}$ geprikkeld en worden alle hartcellen aangezet om te contraheren. Een synchrone contractie van het hart is van belang voor een normale pompfunctie. Wanneer de geleiding van de prikkel over het hart echter gestoord is, bijv. door een ontsteking of door een afgestorven gedeelte, dan vindt er op sommige platsen vertraagde of zelfs helemaal geen prikkeling plaats en als gevolg daarvan zal de contractie ook gestoord of afwezig zijn. Verstoringen van de normale impulsvoortplanting in het hart vormen het onderwerp van dit proefschrift.

In hoofdstuk I wordt een historisch overzicht gegeven van de studies die verricht zijn naar de normale en abnormale geleiding van de electrische impuls in de hartspier. Abnormale geleiding kan niet alleen aanleiding geven tot vertraagde contractie van de hartspier maar het is ook mogelijk gebleken dat de impuls gevangen raakt in een cirkelvormig pad (reentry). In een dergelijk circuit kan de impuls dan eindeloos ronddraaien (cirkelgeleiding). In deze situatie vertoont het hart niet meer zijn normale ritme, maar wordt overheerst door de omlooptijd van deze cirkelbeweging (tachycardie). Een dergelijk mechanisme ligt ten grondslag aan een groot aantal ritmestoornissen die het hart kan treffen.

Voor het ontstaan van een ritmestoornis op basis van cirkelgeleiding moet er aan twee voorwaarden worden voldaan. Ten eerste moet de geleiding van de electrische impuls ergens in het hart gestopt worden (geleidingsblok). Ten gevolge van deze locale verstoring zal de impuls zich om dit blokgebied heen wentelen en zal dan na verloop van tijd weer de plek van het geleidingsblok bereiken. Ten tweede moet het oorspronkelijk geactiveerde weefsell weer prikkelbaar zijn. Of dit zo is hangt af van: A) van de hersteltijd die elke hartcel nodig heeft om na een voorafgaande activatie weer prikkelbaar te worden (refractaire periode) en B) van 
de geleidingssnelheid wan de impuls rondom het blokgebied. In geval van een lange refractaire periode of van een hoge voortplantingssnelheid zal het punt van oorsprong te snel bereikt worden, het weefsel is dan nog niet prikkelbaar en de impuls dooft uit. Wanneer de refractaire periode echter kort genoeg is of de voortplantingssnelheid voldoende traag, dan kan het weefsel weer geactiveerd worden, waardoor reentry plaatsvindt.

De combinatie van refractaire periode en geleidingssnelheid kan samengevat worden in de golflengte van de electrische impuls (golflengte $=$ refractaire periode $x$ geleidingssnelheid). Onder normale omstandigheden is de golflengte lang genoeg en is reentry onmogelijk. Onder bepaalde omstandigheden kan de golflengte echter korter worden, en is reentry in principe mogelijk. Of reentry ook daadwerkelijk ontstaat hangt dan nog af van het initierend moment; het ontstaan van een lokaal geleidingsblok.

Het onderzoek naar de geleidingseigenschappen van het hart vereist een techniek die beschreven wordt in hoofdstuk III. Het moment van activatie op een willekeurige plaats in de hartspier kan worden gemeten door op die plaats met een electrode het activatiesignaal te registreren. Om informatie over de geleidingseigenschapen te verkrijgen is een groot aantal van dergelijke electroden nodig die het activatie-signaal tegelijkertijd en op verschillende plaatsen registreren. In deze studie werd een opname systeem gebruikt, dat het mogelijk maakt om 192 signalen van de oppervlakte van de hartspier te registreren.

Het onderzoek werd verricht aan de geisoleerde linker boezem van het konijnen hart. Met dit preparaat is het mogelijk om de normale en de abnormale geleidings eigenschappen gedurende vele uren te bestuderen. Daartoe wordt het preparaat omspoeld met een voedingsvloeistof. Het is zelfs mogeljk gebleken om in dit kleine preparaat van 15 bij $25 \mathrm{~mm}$ ritmestoornissen op te wekken. Wanneer bijvoorbeeld het kalium- of het zuurstofgehalte van de voedingsvloeistof wordt verlaagd, kunnen tachycardieen makkelijk opgewekt worden. Met behulp van het multipele registratie systeem is het mogelijk om de geleiding van de impuls tijdens het initieren van deze ritmestoornissen te onderzoeken.

In hoofdstuk IV wordt het mechanisme van de ritmestoornissen, opgewekt in laag kalium, beschreven. De meeste tachycardieen bleken te berusten op cirkelgeleiding, terwijl een klein aantal veroorzaakt werd door spontane impulsvorming. Zoals gezegd zijn er twee mogelijke oorzaken voor het makkelijker opwekken van ritmestoornissen op basis van cirkelgeleiding; een korte golflengte of een toegenomen inhomogeniteit in geleiding. In laag kalium bleek 
de golflengte sterk te verkorten, terwijl er geen tekenen voor toegenomen inhomogeniteit in geleiding gevonden konden worden.

In hoofdstuk $V$ zijn de ritmestoornissen die opgewekt konden worden in hypoxie behandeld. Ook hier bleken de meeste ritmestoornissen gebaseerd te zijn op cirkelgeleiding. In tegenstelling tot de situatie in laag kalium echter bleek de golflengte in hypoxie niet wezenlijk korter te worden. Wel kregen wij de indruk dat de geleiding in hypoxie inhomogener was dan onder normale omstandigheden. Om deze indruk in maat en getal uit te drukken is in hoofdstuk VI een eerste poging ondernomen om de hoeveelheid geleidingsblok te tellen. Het daarbij gebruikte principe is eenvoudig. $\mathrm{Bij}$ een redelijke geleidingssnelheid mag men werwachten dat het tijdsverschil tussen de activatie van naburige registratie electroden, met een onderlinge afstand van $1.0 \mathrm{~mm}$, ongeveer $0-7$ milliseconden bedraagt. Wanneer het tijdsverschil echter meer dan 10 milliseconden is, wordt dit veroorzaakt doordat er een geleidingsblok tussen de twee registratie electroden aanwezig was, waardoor de electrische impuls een omweg moest maken om de naburige electrode te kunnen bereiken. Met behulp van dit principe werd het aantal electrodeparen geteld dat meer dan 10 milliseconden verschil vertoonde. In hypoxie bleek, vergeleken met de uitgangssituatie, het aantal plaatsen met geleidingsblok sterk toe te nemen

Het ontstaan van een geleidingsblok is slechts een symptoom van inhomogeniteit in geleiding. Het zou wenselijk zijn een methode te ontwikkelen waarmee de totale geleidingseigenschappen in kaart gebracht kon worden. Een dergelijke methode wordt beschreven in hoofdstuk VII. Ook nu zijn wij uitgegaan van het verschil in activatietijden gemeten tussen naburige registratie electroden. Een dergelijk verschil is de uitdrukking van de mate waarin beide plaatsen ten opzichte van elkaar in fase geprikkeld worden. Wanneer beide plaatsen simultaan geactiveerd worden is het faseverschil tussen beide plaatsen nul en is er geen sprake van inhomogene geleiding. Wanneer echter het verschil in activatietijd tussen beide naburige plaatsen groter wordt, neemt het faseverschil toe en daarmee de mate van inhomogene geleiding. Het voordeel van deze fase methode is dat de locale faseverschillen in een kaart afgedrukt kunnen worden, waardoor de spatiele verdeling van de geleidingseigenschappen duidelijk wordt (fase map). De plaatsen met een sterk inhomogene geleiding vallen op door hoge faseverschillen, terwijl de gebieden die homogeen geactiveerd worden lage faseverschillen vertonen. Het is nu ook mogelijk een index van de inhomogeniteit in geleiding te berekenen. Hierdoor kunnen de geleidingseigenschappen van eenzelfde stuk hartweefsel onder 
verschillende omstandigheden vergeleken worden.

Een aantal toepassingen van de fase methode wordt in hoofdstuk VII beschreven. Het is bijwoorbeeld duidelijk geworden dat de inhomogeniteit in geleiding toenam wanneer, na een normalle hartslag, een extra vroege prikkel gegeven werd (premature slag). Inhomogeniteit in geleiding nam nog sterker toe wanneer meer dan een premature prikkel gegeven werd en de hoogste mate van inhomogeniteit werd gevonden wanneer het hartweefsel met een snel ritme gedreven werd. Een analyse van de ruimtelijke verdeling van geleidingseigenschappen leverde nog een essentieel verschil op tussen de activatie tijdens een premature slag en tijdens snel drijven. Tijdens premature activatie bleek de inhomogeniteit in geleiding uitsluitend voor te komen in een gebied van 6 millimeters rond de prikkelplaats. Tijdens snel drijven bleek de inhomogeniteit in geleiding echter over het hele preparaat te kunnen voorkomen en had de plaats van prikkeling hierop geen invloed.

Met de methode van "fase mapping" bleek het ook mogelijk om de mate van inhomogeniteit in geleiding tijdens het initieren en continuering van een ritmestoornis te kwantificeren. $\mathrm{De}$ potentiele plaatsen van reentry werden onthuld door hoge faseverschillen. De hoogste waarden voor inhomogene geleiding werden gevonden tijdens het initieren van de tachycardie. Was het cirkelpad eenmaal ingesteld, dan nam de inhomogeniteit in geleiding weer af en was alleen nog maar afhankelijk van de frekwentie van de ritmestoornis.

Concluderend kunnen wij stellen dat de methode van fase mapping het ons mogelijk maakt een van de factoren voor het ontstaan van cirkelgeleiding te meten. Met deze methode is het in principe ook mogelijk geworden om de kwaliteit van geleiding in het hartweefsel van patienten te visualiseren en te kwantificeren. Uiteindelijk kan dit leidden tot een betere behandeling van patienten die bedreigd worden door ritmestoornissen op basis van cirkelgeleiding. 


\section{NAWOORD}

Een proefschrift is gelukkig nog steeds een verhandeling als proeve van bekwaamheid. Deze bekwaamheid komt iemand niet aangevlogen. Het is het resultaat van vele jaren van begeleiding, hulp en toewijding van vrienden en collega's. Mede aan hun inzet is het te danken dat het tot deze proeve is gekomen.

Prof. Allessie, beste Maurits, vanaf het moment dat je mij meenam naar Maastricht, nu 12 jaar geleden, heb je mij onafgebroken wegwijs gemaakt in het wetenschappelijk onderzoek. Dit proefschrift is vooral een weerspiegeling van jouw begeleiding. Jouw werkwijze heeft niet alleen zijn weerslag gehad op mijn wetenschappelijke vorming, maar heeft ook stimulerend gewerkt op mijn eigen persoonlijke ontwikkeling. Ik hoop daarom dat wij nog vele jaren vruchtbaar zullen mogen samenwerken.

Prof. Bonke, beste Vic, ik ben je veel verschuldigd. Vanaf onze vroegste contacten, het was nog in Amsterdam, heb je me gesteund en aangemoedigd. Zowel in het onderzoek als ook in het onderwijs heb je mij steeds de juiste weg gewezen. Hiervoor blijf ik je erkentelijk.

Prof. Wit, beste Andy, onze eerste contacten stammen van je eerste sabbatical in Maastricht. In de loop der jaren zijn ze uitgegroeid tot een vriendschap die verder reikt dan ons gemeenschappelijk vakgebied. De gastvrijheid die ik bij jou en Peggy in Massapequa meerdere malen heb mogen ervaren strekken Marijke en mij tot voorbeeld.

Prof. Janse, beste Giel, jou wil ik bedanken omdat je referent van dit proefschrift hebt willen zijn. Menigmaal hebben wij elkaar bij gelegenheid van verschillende congressen ontmoet. Voor je manier van werken, met name tijdens onze jaarlijke AmsterdamMaastricht bijeenkomsten, heb ik grote bewondering gekregen.

Prof. Wellens, wil ik dank zeggen voor zijn niet aflatende belangstelling en steun voor het onderzoek op het gebied van ritmestoornissen verricht aan diermodellen. Dat $u$ dit proefschrift hebt willen referen is daar slechts een uiting van.

Prof. Reneman, beste Rob, ik wil je niet uitsluitend danken voor je lidmaatschap van de leescommissie. Vooral als architect van het onderzoek van de vakgroep Fysiologie heb je het mij mogelijk gemaakt om in alle rust aan het onderwerp van dit proefschrift te 
werken. Dank ook voor je luisterend oor en de belangstelling die je altijd voor mij had.

Een groot deel van de werkvreugde die ik in de afgelopen jaren heb mogen genieten heb ik te danken aan degenen die mijn kamer met mij deelden: Joep Smeets, Wieke Rensma en Martin Schalij. De wijze waarop jullie steeds als klankbord en toetssteen fungeerden heeft veel voor mij betekend.

Voor het dagelijks werk in een electrofysiologisch laboratorium is vakkundige technische ondersteuning onontbeerlijk. Jan Corsel en later Jan Hollen hebben daar op voortreffelijke wijze voor gezorgd. Henk Jas, computer-expert van VAX tot PC, wil ik danken voor de snelle wijze waarop hij mijn vaak hectische en onduidelijke vragen wist te vertalen en beantwoorden.

Drie studenten, Charles Kirchhof, Harry Gelissen en Rein Rutten heb ik, in het kader van hun keuze-stage op het onderwerp inhomogeniteit in geleiding, mogen begeleiden. Hen wil ik danken voor het enthousiasme en de werkkracht waarmee zij die stage aanpakten. Dat Charles Kirchhof bij onze groep is blijven werken doet mij genoegen. Ik wens hem veel succes met zijn eigen proefschrift.

Lucienne de Boer, ondanks de opkomst van tekstverwerkers, is secretariele ondersteuning nog steeds onontbeerlijk. Je deed dat, samen met Jos Heemskerk, Karin van Brussel en Emmy van Roosmalen, altijd op je eigen voortreffelijke wijze en daarvoor wil ik je danken.

De opkomst van de laserprinter heeft de drukkwaliteit van dit proefschrift hopelijk verbeterd. Carin Heymen en Harry Wetzelaer, bedankt dat jullie mij in staat stelde deze nieuwe techniek toe te passen.

Het is onmogelijk om iedereen die in materiele of immateriele zin heeft bijgedragen tot het tot stand komen van dit proefschrift te noemen. Daarom wil ik, tot slot, alle collega's van de vakgroep fysiologie bedanken, als ook de vele vrienden binnen en buiten het BMC en tenslotte mijn familie, die, ondanks de bijna "eeuwigdurende" wachttijd, toch altijd is blijven geloven dat deze proeve van bekwaamheid ooit nog eens tot stand zou komen. 


\section{CURRICULUM VITAE}

12 october 1947 : geboren te Rome.

1966 : HBS-b, R.K. Lyceum, Hilversum.

1966 - 1980 : studie geneeskunde aan de Gemeentelijke Universiteit van Amsterdam.

$1972-1975$ : student-assistent bij de vakgroep Fysiologie, Gemeentelijke Universiteit van Amsterdam.

september $1980 \quad$ : artsdiploma.

$1975-1987$ : wetenschappelijk medewerker vakgroep Fysiologie, Rijksuniversiteit Limburg. 